\title{
Loop Quantum Gravity Phenomenology: Linking Loops to Observational Physics ${ }^{\star}$
}

\author{
Florian GIRELLI ${\dagger^{1} \dagger^{2}}^{2}$ Franz HINTERLEITNER ${ }^{\dagger^{3}}$ and Seth A. MAJOR ${ }^{\dagger^{4}}$ \\ $\dagger^{1}$ Department of Applied Mathematics, University of Waterloo, Waterloo, Ontario, Canada \\ E-mail: fgirelli@uwaterloo.ca \\ $\dagger^{2}$ University Erlangen-Nuremberg, Institute for Theoretical Physics III, Erlangen, Germany \\ $\dagger^{3}$ Department of Theoretical Physics and Astrophysics, Faculty of Science \\ of the Masaryk University, Kotlářská 2, 61137 Brno, Czech Republic \\ E-mail: franz@physics.muni.cz \\ $\dagger^{4}$ Department of Physics, Hamilton College, Clinton NY 13323, USA \\ E-mail: smajor@hamilton.edu
}

Received May 30, 2012, in final form December 03, 2012; Published online December 13, 2012 http://dx.doi.org/10.3842/SIGMA.2012.098

\begin{abstract}
Research during the last decade demonstrates that effects originating on the Planck scale are currently being tested in multiple observational contexts. In this review we discuss quantum gravity phenomenological models and their possible links to loop quantum gravity. Particle frameworks, including kinematic models, broken and deformed Poincaré symmetry, non-commutative geometry, relative locality and generalized uncertainty principle, and field theory frameworks, including Lorentz violating operators in effective field theory and non-commutative field theory, are discussed. The arguments relating loop quantum gravity to models with modified dispersion relations are reviewed, as well as, arguments supporting the preservation of local Lorentz invariance. The phenomenology related to loop quantum cosmology is briefly reviewed, with a focus on possible effects that might be tested in the near future. As the discussion makes clear, there remains much interesting work to do in establishing the connection between the fundamental theory of loop quantum gravity and these specific phenomenological models, in determining observational consequences of the characteristic aspects of loop quantum gravity, and in further refining current observations. Open problems related to these developments are highlighted.
\end{abstract}

Key words: quantum gravity; loop quantum gravity; quantum gravity phenomenology; modified dispersion relation

2010 Mathematics Subject Classification: 83-02; 83B05; 83C45; 83C47; 83C65

\section{Contents}

1 Introduction $\quad 2$

2 Discreteness of LQG geometric operators 5

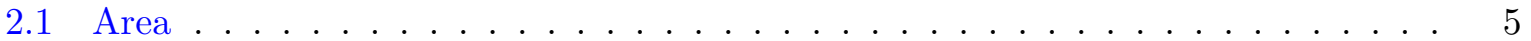

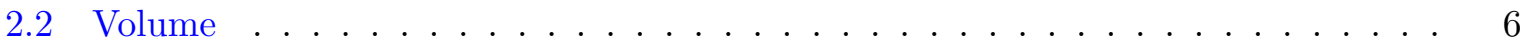

2.3 Length . . . . . . . . . . . . . . . . . . . . . . . . 8

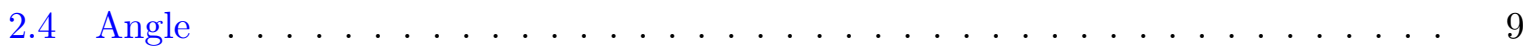

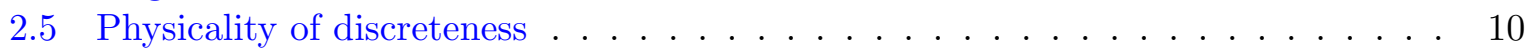

2.6 Local Lorentz invariance and $\mathrm{LQG} \ldots \ldots$

${ }^{\star}$ This paper is a contribution to the Special Issue "Loop Quantum Gravity and Cosmology". The full collection is available at http://www.emis.de/journals/SIGMA/LQGC.html 
3 Quantum particle frameworks $\quad 12$

3.1 Relativistic particles and plane-waves . . . . . . . . . . . . . . . . . . 12

3.2 Introducing Planck scales into the game: modified dispersion relations . . . . . . 14

3.3 Arguments linking modified dispersion relations and LQG . . . . . . . . . . . 15

3.3.1 MDR from weave states . . . . . . . . . . . . . . . . . 15

3.3.2 Quantum field theory in curved space from LQG . . . . . . . . . . . . 18

3.3.3 MDR from Hamilton-Jacobi theory . . . . . . . . . . . . . . . 19

3.4 Broken Poincaré symmetries: Finsler geometry . . . . . . . . . . . . . . . 21

3.5 Non-linear realization of Poincaré symmetries . . . . . . . . . . . . . . . . 23

3.6 Modified reference frame . . . . . . . . . . . . . . . . . . . 25

3.7 Non-commutative space-time . . . . . . . . . . . . . . . . . . . 26

3.8 Relative locality . . . . . . . . . . . . . . . . . . . . . . . . . 31

3.9 Generalized uncertainty principle . . . . . . . . . . . . . . . 33

3.10 QG decoherence . . . . . . . . . . . . . . . . . . 34

4 Quantum field theory frameworks $\quad 34$

4.1 Constraints on Lorentz violation with effective field theory . . . . . . . . . . 35

4.1.1 Physical effects giving current constraints . . . . . . . . . . . . . 37

4.1.2 Kinematic constraints arising from birefringence . . . . . . . . . . . . 37

4.1.3 Dynamical constraints arising from photon stability . . . . . . . . . 37

4.1 .4 Neutrino physics . . . . . . . . . . . . . . . . . . . 39

4.1.5 Current bounds from astrophysical observation: summary . . . . . . . . 40

4.1.6 The naturalness problem . . . . . . . . . . . . . . . . 40

4.1.7 Generalizations and prospects for improved constraints . . . . . . . . . . 42

4.2 EFT Phenomenology without Lorentz violation:

a combinatoric lever arm . . . . . . . . . . . . . . . . . 43

4.3 Non-commutative field theory . . . . . . . . . . . . . . . . . . . . 44

4.3 .1 Overview . . . . . . . . . . . . . . . . . . 44

4.3.2 Relating non-commutative field theory and spinfoam models . . . . . . 49

5 Loop quantum cosmology $\quad \mathbf{5 0}$

5.1 Holonomy corrections . . . . . . . . . . . . . . . . . . . 53

5.2 Inverse volume corrections . . . . . . . . . . . . . . . . . . 53

5.3 Dynamics and lattice refinement . . . . . . . . . . . . . . . . . 55

5.4 Loop Quantum Cosmology: possible observational consequences . . . . . . . . . 57

5.4 .1 Scalar and tensor perturbations . . . . . . . . . . . 58

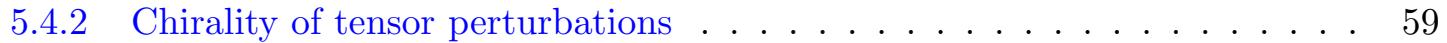

5.5 Phenomenology of black hole evaporation . . . . . . . . . . . . . . 59

$\begin{array}{lll}6 & \text { Conclusions } & 60\end{array}$

$\begin{array}{ll}\text { A Elements of LQG } & 60\end{array}$

$\begin{array}{ll}\text { References } & 63\end{array}$

\section{Introduction}

Twenty five years ago Ashtekar, building on earlier work by Sen, laid the foundations of Loop Quantum Gravity (LQG) by reformulating general relativity (GR) in terms of canonical connection and triad variables - the "new variables". The completion of the kinematics - the quantum theory of spatial geometry - led to the prediction of a granular structure of space, 
described by specific discrete spectra of geometric operators, area [228], volume [162, 169,228], length $[50,171,253]$ and angle [187] operators. The discreteness of area led to an explanation of black hole entropy $[56,158,160,224]$ (see [83] for a recent review). Although granularity in spatial geometry is predicted not only by LQG, but also by some string theory and non-commutative geometry models, the specific predictions for the spectra of geometry operators bears the unique stamp of LQG.

Quantum effects of gravity are expected to be directly perceptible at distances of the order of the Planck length, about $10^{-35} \mathrm{~m}$, in particle processes at the Planck energy, about $10^{28} \mathrm{eV}$ $(c=1)$, and at a Planck scale density. With the typical energy $M_{\mathrm{QG}}$ for quantum gravity (QG) assumed to be of the order of the Planck energy, there are fifteen orders of magnitude between this energy scale and the highest attainable center-of-mass energies in accelerators and, in the Earth's frame, eight orders of magnitude above the highest energy cosmic rays. So fundamental quantum theories of gravity and the realm of particle physics appear like continents separated by a wide ocean. (Although, if the world has large extra dimensions, the typical energy scale of quantum gravity may be significantly lower.) The situation is worsened by the fact that none of the tentative QG theories has attained such a degree of maturity that would allow to derive reliable predictions of such a kind that could be extrapolated to our "low-energy" reality. It would appear that there is little hope in directly accessing the deep quantum gravity regime via experiment. One can hope, however, to probe the quantum gravity semi-classical regime, using particle, astrophysical and cosmological phenomena to enhance the observability of the effects.

In spite of this discouraging perspective, over one decade ago a striking paper by AmelinoCamelia et al. [21] on quantum gravity phenomenology appeared. The paper was based on a plausibility argument: The strong gravity regime is inaccessible but quantum gravity, as modeled in certain models of string theory and, perhaps, in the quantum geometry of LQG, has a notion of discreteness in its very core. This discreteness is understood to be a genuine property of space, independent of the strength of the actual gravitational field at any given location. Thus it may be possible to observe QG effects even without strong gravitational field, in the flat space limit. In [21] the authors proposed that granularity of space influences the propagation of particles, when their energy is comparable with the QG energy scale. Further, the assumed invariance of this energy scale, or the length scale, respectively, are in apparent contradiction with special relativity (SR). So it is expected that the energy-momentum dispersion relation could be modified to include dependence on the ratio of the particle's energy and the QG energy. At lowest order

$$
E \simeq p+\frac{m^{2}}{2 p} \pm \xi \frac{E^{2}}{M_{\mathrm{QG}}}
$$

with the parameter $\xi>0$ of order unity. Relations like (1.1) violate, or modify, local Lorentz invariance (LLI). According to the sign in (1.1), the group velocity of high-energy photons could be sub- or super-luminal, when defined in the usual way by $\partial E / \partial p$. Like with all QG effects, the suppression of Lorentz invariance violation by the ratio of the particle energy to the QG energy may appear discouraging at first sight. To have a chance to detect an effect of the above modification, we need an amplification mechanism, or "lever arm".

The authors of [21] showed that if the tiny effect on the speed of light accumulates as high energy photons travel cosmic distances, the spectra of $\gamma$ ray bursts (GRB) would reveal an energy-dependent speed of light through a measurable difference of the time of arrival of high and low energy photons. Due to different group velocities $v=\partial \omega / \partial k \simeq 1+\xi k / M_{\mathrm{QG}}$, photons emitted at different momenta, $k_{1}$ and $k_{2}$, would arrive at a distant observer (at distance $D$ ) at times separated by the interval $\Delta t \simeq \xi\left(k_{2}-k_{1}\right) D / M_{\mathrm{QG}}$. Distant sources of $\gamma$-ray photons are the best for this test. Despite the uncertainties concerning the physics of the psroduction of 
such $\gamma$-rays, one can place limits on the parameter $\xi_{0}$. The current strongest limit is $\xi \lesssim 0.8$ reported by the Fermi Collaboration using data from the $\gamma$-ray burst GRB 090510 [2]. This is discussed further in Section 4.1.1.

In the years following this work, the nascent field of QG phenomenology developed [18] from ad hoc effective theories, like isolated isles lying between the developing QG theories and reality, linked to the former ones loosely by plausibility arguments. Today the main efforts of QG phenomenology go in two directions: to establish a bridge between the intermediate effective theories and the fundamental QG theory and the refinement of observational methods, through new effective theories and experiments that could shed new light on QG effects. These are exceptionally healthy developments for the field. The development of physical theory relies on the link between theory and experiment. Now these links between current observation and quantum gravity theory are possible and under active development.

The purpose of this review on quantum gravity phenomenology is three-fold. First, we wish to provide a summary of the state of the art in LQG phenomenology and closely related fields with particular attention to theoretical structures related to LQG and to possible observations that hold near-term promise. Second, we wish to provide a road map for those who wish to know which physical effects have been studied and where to find more information on them. Third, we wish to highlight open problems.

Before describing in more details what is contained in this review, we remind the reader that, of course, the LQG dynamics remains open. Whether a fully discrete space-time follows from the discreteness of spatial geometry is a question for the solution to LQG dynamics generated by the master constraint, the Hamiltonian constraint operator, and/or spin foam models [226,257]. Nevertheless if the granular spatial geometry of LQG is physically correct then it must manifest itself in observable ways. This review concerns the various avenues in which such phenomenology is explored.

The contents of our review is organized in the the following four sections:

Section 2: A brief introduction to the geometric operators of LQG, area, volume, length and angle, where discreteness shows up.

Section 3: An overview of particle effective theories of the type introduced above. In this section we review particle kinematics, discuss arguments in LQG that lead to modified dispersion relations (MDR) like the kind (1.1) and discuss models of symmetry deformation. The variety of models underlines their loose relation to fundamental theories such as LQG.

Section 4: A brief review of field theories leading to phenomenology including effective field theory with Lorentz symmetry violation and non-commutative field theory. The effective field theories incorporate MDR and contain explicit Lorentz symmetry violation. A model with LLI is discussed and, in the final part, actions for field theories over non-commutative geometries are discussed.

Section 5: A brief discussion of loop quantum cosmology and possible observational windows. Cosmology is a promising observational window and a chance to bridge the gap between QG and reality directly, without intermediate effective theories: The cosmic microwave and gravitational wave background fluctuations allow a glimpse into the far past, closer to the conditions when the discreteness of space would play a more dominant role.

The Planck length is given when the Compton length is equivalent to the Schwarzschild length, $\ell_{\mathrm{P}}:=\sqrt{\hbar G / c^{3}} \simeq 1.6 \times 10^{-35} \mathrm{~m}$. Similarly, the Planck mass is given when the Compton mass is equivalent to the Schwarzschild mass, $M_{\mathrm{P}}:=\sqrt{\hbar c / G} \simeq 1.2 \times 10^{28} \mathrm{eV} / \mathrm{c}^{2}$. These conditions mean that at these scales, quantum effects are comparable to gravitational effects. The usual physical argument, which [88] made more rigorous, is that to make a very precise measurement of a distance, we use a photon with very high energy. The higher the precision, 
the higher the energy will be in a small volume of space, so that gravitational effects will kick in due to a large energy density. When the volume is small enough, i.e. the precision very high, the energy density is so large that a black hole is created and the photon can not come back to the observer. Hence there is a maximum precision and a notion of minimum length $\ell_{\mathrm{P}}$. This argument goe s beyond the simple application of dimensional analysis of the fundamental scales of the quantum gravitational problem, $c, G$, $\hbar$, and $\Lambda$, the cosmological constant. In the remainder of this review, except where it could lead to confusion, we set $c=1$ and denote the Planck scale mass by $\kappa$ so that the Planck scale $\kappa=M_{\mathrm{P}}=\frac{1}{\ell_{\mathrm{P}}}$ can be interpreted as Planck momentum $\kappa=M_{\mathrm{P}} c$, Planck energy $\kappa=M_{\mathrm{P}} c^{2}$ or Planck rest mass $\kappa=M_{P}$.

\section{Discreteness of LQG geometric operators}

Loop quantum gravity hews close to the classical theory of general relativity, taking the notion of background independence and apparent four dimensionality of space-time seriously. The quantization has been approached in stages, with work on kinematics, the quantization of spatial geometry, preceding the dynamics, the full description of space-time. The kinematics, all but unique, reveals a picture of quantized space. This quantization, this granularity, inspired the phenomenological models in this review and is the subject of this section. We focus on the geometric observables here. For a brief review of the elements of LQG and the new variables see Appendix A.

In LQG the operators representing area, angle, length, and volume have discrete spectra, so discreteness is naturally incorporated into LQG. This fundamental discreteness predicted by LQG, must be at some level physically manifest. Much of the work in phenomenology related to LQG has been an effort to link up this predicted discreteness with possible observational contexts. In later sections we will introduce a fundamental length or energy scale, adding terms to the effective action for particles, exploring effects of an minimum area on cosmological models, and studying the affects of underlying combinatorics on geometric quantities.

\section{$2.1 \quad$ Area}

Classically the area of a two-dimensional surface is the integral over the square root of the determinant of the induced two-dimensional metric. Thus,

$$
A(\mathcal{S})=\int_{\mathcal{S}} \sqrt{n_{a} E^{a i} n_{b} E^{b}} \mathrm{~d}^{2} \sigma .
$$

(For details see $[38,226]$.) The operators related to $E^{a}{ }_{i}$, namely the flux operators $E_{i}(\mathcal{S})$, associated to $\mathcal{S}$, have a Lie algebra index and so are not gauge invariant. Nor does its "square" $E^{2}(\mathcal{S}):=E^{i}(\mathcal{S}) E_{i}(\mathcal{S})$ give rise to a gauge-invariant operator in general, because the integration over $\mathcal{S}$ complicates the transformation properties, when there are more than one intersection of a spin network $(\mathrm{SNW})$ graph $\gamma$ with $\mathcal{S}$. Its action on a single link intersecting $\mathcal{S}$, however, is simple: Each $E_{i}$ inserts an $\mathfrak{s u}(2)$ generator $\tau_{i}^{(j)}$ into the corresponding holonomy, which results in the Casimir operator of $\mathrm{SU}(2)$ in the action of $E^{2}(\mathcal{S})$, namely

$$
\sum_{i} \tau_{i}^{(j)} \tau_{i}^{(j)}=j(j+1) \cdot \mathbb{1}
$$

To make use of this simple result in the case of extended graphs intersecting $\mathcal{S}$, one partitions the surface into $n$ small surfaces $\mathcal{S}_{i}$, such that each of them contains not more than one intersection point with the given graph, and then takes the sum over the small sub-surfaces,

$$
A(\mathcal{S}):=\lim _{n \rightarrow \infty} \sum_{i=1}^{n} \sqrt{E^{2}\left(\mathcal{S}_{i}\right)} .
$$


This defines the area operator. Area can be equally well defined in a combinatorial framework as discussed in Appendix A.

The action on a SNW function $\Psi_{\Gamma}$ is

$$
A(\mathcal{S})\left|\Psi_{\Gamma}\right\rangle=\frac{8 \pi \gamma}{\kappa^{2}} \sum_{p \in \Gamma \cap \mathcal{S}} \sqrt{j_{p}\left(j_{p}+1\right)}\left|\Psi_{\Gamma}\right\rangle,
$$

where $j_{p}$ is the spin, or color, of the link that intersects $\mathcal{S}$ at $p$ and $\gamma$ is the Barbero-Immirizi parameter. The area operator acts only on the intersection points of the surface with the SNW graph, $\gamma \cap \mathcal{S}$ and so gives a finite number of contributions. SNW functions are eigenfunctions. The eigenvalues are obviously discrete. The quanta of area live on the edges of the graph and are the simplest elements of quantum geometry. There is a minimal eigenvalue, the so-called area gap, which is the area when a single edge with $j=1 / 2$ intersects $\mathcal{S}$,

$$
\Delta A=4 \sqrt{3} \pi \hbar G c^{-3} \sim 10^{-70} \mathrm{~m}^{2} .
$$

This is the minimal quantum of area, which can be carried by a link.

The eigenvalues (2.1) form only the main sequence of the spectrum of the area operator. When nodes of the SNW lie on $\mathcal{S}$ and some links are tangent to it the relation is modified, see $[38,226]$. The important fact, independent of these details, is that discreteness of area with the SNW links, carrying its quanta, comes out in a natural way. The interpretation of discrete geometric eigenvalues as observable quantities goes back to early work in [222]. This discreteness made the calculation of black hole entropy possible by counting the number of microstates of the gravitational field that lead to a given area of the horizon within some small interval.

Intriguingly, area operators acting on surfaces that intersect in a line fail to commute, when SNW nodes line in that intersection [32]. One may see this as resulting from the commutation relations among angular momentum operators in the two area operators. Recently additional insight into this non-commutativity comes from the formulation of discrete classical phase space of loop gravity, in which the flux operators also depend on the connection [94].

Another, inequivalent, form of the area operator was proposed in [159]. This operator, $\tilde{A}_{\mathcal{S}}$, is based on a non gauge-invariant expression of the surface metric. Fix a unit vector is the Lie algebra, $r^{i}$ then the classical area may be expressed as the maximum value of

$$
\tilde{A}_{\mathcal{S}}=\int_{\mathcal{S}} \sqrt{n_{a} E^{a i} r_{i}} \mathrm{~d}^{2} \sigma
$$

where the maximum is obtained by gauge rotating the triad. On the quantum mechanical side this value is the maximum magnetic quantum number, simply $j$ so the spectrum is simply

$$
\tilde{A}(\mathcal{S})\left|\Psi_{\Gamma}\right\rangle=\frac{8 \pi \gamma}{\kappa^{2}} \sum_{p \in \Gamma \cap \mathcal{S}} j_{p}\left|\Psi_{\Gamma}\right\rangle
$$

This operator, frequently used in the spin foam context, is particularly useful in systems with boundary such as where gauge invariance might be (partially) fixed.

\subsection{Volume}

Like the area of a surface, the volume of a region $\mathcal{R}$ in three-dimensional space, the integral of the square root of the determinant of the metric, can be expressed in terms of densitized triads,

$$
V(\mathcal{R})=\int_{\mathcal{R}} \mathrm{d}^{3} x \sqrt{\frac{1}{3 !}\left|\epsilon_{a b c} \epsilon^{i j k} E^{a}{ }_{i}(x) E^{b}{ }_{j}(x) E^{c}{ }_{k}(x)\right|} .
$$


Regularizations of this expression consist of partitioning the region under consideration into cubic cells in some auxiliary coordinates and constructing an operator for each cell. The cells are shrunk to zero coordinate volume. This continuum limit is well-defined, thanks to discreteness reached when the cells are sufficiently small, but finite. Readers interested in precisely how this is done should consult $[39,228]$.

There are, primarily, two definitions of the operator, one due to Rovelli and Smolin (RS) [228] and the other due to Ashtekar and Lewandowski (AL) [39]. Here we present the AL volume operator of [39], presented also in [257]. For a given SNW function based on a graph $\Gamma$, the operator $\hat{V}_{\mathcal{R}, \Gamma}$ of the volume of a region $\mathcal{R}$ acts nontrivially only on (at least four-valent [169]) vertices in $\mathcal{R}$. According to the three triad components in (2.2), which become derivatives upon quantization, in the volume operator three derivative operators $\hat{X}_{v, e_{I}}^{i}$ act at every node or vertex $v$ on each triple of adjacent edges $e_{I}$,

$$
\hat{V}_{\mathcal{R}, \Gamma}=\left(\frac{\ell_{\mathrm{P}}}{2}\right)^{3} \sum_{v \in \mathcal{R}} \sqrt{\left|\frac{i}{3 ! \cdot 8} \sum_{I, J, K} s\left(e_{I}, e_{J}, e_{K}\right) \epsilon_{i j k} \hat{X}_{v, e_{I}}^{i} \hat{X}_{v, e_{J}}^{j} \hat{X}_{v, e_{K}}^{k}\right|} .
$$

Dependence on the tangent space structure of the embedding is manifest in $s\left(e_{I}, e_{J}, e_{K}\right)$. This is $+1(-1)$, when $e_{I}, e_{J}$, and $e_{K}$ are positive (negative) oriented, and is zero when the edges are coplanar. The action of the operators $\hat{X}_{v, e_{I}}^{i}$ on a SNW function $\Psi_{\Gamma}=\psi\left(h_{e_{1}}(A), \ldots, h_{e_{N}}(A)\right)$ based on the graph $\Gamma$ is

$$
\hat{X}_{v, e_{I}}^{i} \Psi_{\Gamma}(A)=i \operatorname{tr}\left(h_{e_{I}}(A) \tau^{i} \frac{\partial \psi}{\partial h_{e_{I}}(A)}\right),
$$

when $e_{I}$ is outgoing at $v$. This is the action of the left-invariant vector field on $\mathrm{SU}(2)$ in the direction of $\tau^{i}$; for ingoing edges it would be the right-invariant vector field.

Given the "triple-product" action of the operator (2.3), vertices carry discrete quanta of volume. The volume operator of a small region containing a node does not change the graph, nor the colors of the adjacent edges, it acts in the form of a linear transformation in the space of intertwiners at the vertex for given colors of the adjacent edges. It is then this space of intertwiners that forms the "atoms of quantum geometry".

The complete spectrum is not known, but it has been investigated [66-68, 85, 198, 254]. In the thorough analysis of $[66,67]$, Brunnemann and Rideout showed that the volume gap, i.e. the lower boundary for the smallest non-zero eigenvalue, depends on the geometry of the graph and doesn't in general exist. In the simplest nontrivial case, for a four-valent vertex, the existence of a volume gap is demonstrated analytically.

The RS volume operator [228] (see also [226]) differs from the AL operator outlined above. In this definition the densitized triad operators are integrated over surfaces bounding each cell with the results that the square root is inside the sum over $I, J, K$ and the orientation factor $s\left(e_{I}, e_{J}, e_{K}\right)$ is absent. Due to the orientation factor the volume of a node with coplanar tangent vectors of the adjacent links is zero, when calculated with the AL operator, whereas the RS operator does not distinguish between coplanar and non-coplanar links.

The two volume operators are inequivalent, yielding different spectra. While the details of the spectra of the Rovelli-Smolin and the Ashtekar-Lewnadowski definitions of the volume operator differ, they do share the property that the volume operator vanishes on all gauge invariant trivalent vertices $[168,169]$.

According to an analysis in $[104,105]$ the AL operator is compatible with the flux operators, on which it is based, and the RS operator is not. On the other hand, thanks to its topological structure the RS volume does not depend on tangent space structure; the operator is 'topological' in that is invariant under spatial homeomorphisms. It is also covariant also under "extended diffeomorphisms", which are everywhere continuous mappings that are invertible everywhere 
except at a finite number of isolated points; the AL operator is invariant under diffeomorphisms. For more on the comparison see [226, 257].

Physically, the distinction between the two operators is the role of the tangent space structure at SNW nodes. There is some tension in the community over the role of this structure. Recent developments in twisted discrete geometries [98] and the polyhedral point of view [51] may help resolve these issues. It would be valuable to investigate ways in which the tangent space structure, and associated moduli [123], could be observationally manifest.

In [52] Bianchi and Haggard show that the volume spectrum of the 4-valent node may be obtained by direct Bohr-Sommerfeld quantization of geometry. The description of the geometry goes all the way back to Minkowski, who showed that the shapes of convex polyhedra are determined from the areas and unit normals of the faces. Kapovich and Millson showed that this space of shapes is a phase space, and it is this phase space - the same as the phase space of intertwiners - that Bianchi and Haggard used for the Bohr-Sommerfeld quantization. The agreement between the spectra of the Bohr-Sommerfeld and LQG volume is quite good [52].

\subsection{Length}

In constructing the length operator one faces with the challenges of constructing a one-dimensional operator in terms of fluxes and of constructing the inverse volume operator. There are three versions of the length operator. One [253] requires the same trick, due to Thiemann [258], that made the construction of the inverse volume operator in cosmology and the Hamiltonian constraint operator in the real connection representation possible. The second operator [50], due to Bianchi, uses instead a regularization guided by the dual picture in LQG, where one considers (quantum) convex polyhedral geometries dual to SNW nodes, the atoms of quantum geometry. For more discussion on the comparison between these two operators, see [50]. The third operator can be seen to be an average of a formula for length based on area, volume and flux operators [171]. To give a flavor of the construction we will review the first definition based on [253].

Classically the length of a (piecewise smooth) curve $c:[0,1] \rightarrow \Sigma$ in the spatial 3-manifold $\Sigma$ with background metric $q_{a b}$ is given by

$$
L=\int_{0}^{1} \mathrm{~d} t \sqrt{q_{a b}(c(t)) \dot{c}^{a}(t) \dot{c}^{b}(t)}
$$

In LQG the metric is not a background structure, but can be given in terms of the inverse fundamental triad variables,

$$
q_{a b}=\operatorname{det}\left(E^{a}{ }_{i}\right) E_{a}{ }^{i} E_{b}{ }^{i},
$$

that is

$$
q_{a b}=\epsilon_{a c d} \epsilon_{b e f} \epsilon^{i j k} \epsilon^{i m n} \frac{E^{c}{ }_{j} E_{k} E^{e}{ }_{m} E^{f}{ }_{n}}{4 \operatorname{det}\left(E^{g}{ }_{l}\right)} .
$$

The problem is to find an operator equivalent to this complicated non-polynomial expression: any operator version of the denominator would have a huge kernel in the Hilbert space, so that the above expression cannot become a densely defined operator.

Fortunately $q_{a b}$ can be expressed in terms of Poisson brackets of the connection $A_{a}:=A_{a}{ }^{i} \tau_{i}$ $\left(\tau_{i} \in \mathfrak{s u}(2)\right)$ with the volume

$$
q_{a b}=-\frac{1}{8 \pi^{2} G^{2}} \operatorname{tr}\left(\left\{A_{a}, V\right\}\left\{A_{b}, V\right\}\right) .
$$


$V$ can be formulated as a well-defined operator. The connection $A_{a}$, on the other hand, can be replaced by its holonomy, when the curve is partitioned into small pieces, so that the exponent $\int A_{a} \dot{c}^{a}$ of the holonomy is small and higher powers can be neglected in first approximation. The zeroth-order term (which is the unity operator) does not contribute to the Poisson brackets.

The length operator is constructed as a Riemann sum over $n$ pieces of the curve and by inserting the volume operator $\hat{V}$ and replacing the Poisson brackets by $1 / i \hbar$ times the commutators,

$$
\hat{L}_{n}(c)=\ell_{\mathrm{P}} \sum_{i=1}^{n} \sqrt{-8 \operatorname{tr}\left(\left[h_{c}\left(t_{i-1}, t_{i}\right), \hat{V}\right]\left[h_{c}\left(t_{i-1}, t_{i}\right)^{-1}, \hat{V}\right]\right)} .
$$

In the limit $n \rightarrow \infty$ the approximation of $A$ by its holonomy becomes exact.

In [253] it is shown that this is indeed a well-defined operator on cylindrical functions and, due to the occurrence of the volume operator, its action on SNW functions gives rise to nonzero contributions only when the curve contains SNW vertices. As soon as the partition is fine enough for each piece to contain not more than one vertex, the result of $\hat{L}_{n} \Psi$ remains unchanged when the partition is further refined. So the continuum limit is reached for a finite partition.

However this action on SNWs raises a problem. For any given generic SNW a curve $c$ will rarely meet a vertex, so that for macroscopic regions lengths will always be predicted too short in relation to volume and surface areas: $c$ is "too thin". To obtain reasonable results in the classical limit, one combines curves together to tubes, that is two-dimensional congruences of curves with $c$ in the center and with cross-sections of the order of $\ell_{\mathrm{P}}^{2}$. The spectra of the so-constructed tube-operators are purely discrete.

None of the phenomenological models discussed in this review depend on the specific form of the length operator. These have already been compared from the geometric point of view [50]. As with the volume operators it would be interesting to develop phenomenological models that observationally distinguish the different operators.

\subsection{Angle}

The angle operator is defined using a partition of the closed dual surface around a single SNW node into three surfaces, $\mathcal{S}_{1}, \mathcal{S}_{2}, \mathcal{S}_{3}$, the angle operator is defined in terms of the associated flux variables $E^{i}\left(\mathcal{S}_{I}\right)[187]$

$$
\theta_{n}^{(12)}:=\arccos \frac{E^{i}\left(\mathcal{S}_{1}\right) E_{i}\left(\mathcal{S}_{2}\right)}{A\left(\mathcal{S}_{1}\right) A\left(\mathcal{S}_{2}\right)} \text {. }
$$

As is immediately clear from the form of the operator (and dimensional analysis), there is no scale associated to the angle operator. It is determined purely by the state of the intertwiner, the atom of quantum geometry. Deriving the spectrum of the angle operator of equation (2.4) is a simple exercise in angular momentum algebra [187]. Dropping all labels on the intertwiner except those that label the spins originating from one of the three partitions, we have

$$
\begin{aligned}
& \hat{\theta}_{(12)}\left|j_{1} j_{2} j_{3}\right\rangle=\theta_{(12)}\left|j_{1} j_{2} j_{3}\right\rangle \quad \text { with } \\
& \theta_{(12)}=\arccos \left(\frac{j_{3}\left(j_{3}+1\right)-j_{1}\left(j_{1}+1\right)-j_{2}\left(j_{2}+1\right)}{2\left[j_{1}\left(j_{1}+1\right) j_{2}\left(j_{2}+1\right)\right]^{1 / 2}}\right) .
\end{aligned}
$$

where the $j_{i}$ are the spins on the internal graph labeling the intertwiner. As such they can be seen to label "internal faces" of a polyhedral decomposition of the node. For a single partition of the dual surface the angle operators commute. But, reflecting the quantum nature of the atom 
of geometry and the same non-commutativity as for area operators for intersecting surfaces, the angle operators for different partitions do not commute.

As is clear from a glance at the spectrum there are two aspects of the continuum angular spatial geometry that are hard to model with low spin. First, small angles are sparse. Second, the distribution of values is asymmetric and weighted toward large angles. As discussed in [191,239] the asymmetry persists even when the spins are very large.

\subsection{Physicality of discreteness}

A characteristic feature of the above geometric operators is their discrete spectra. It is natural to ask whether it is physical. Can it be used as a basis for the phenomenology of quantum geometry? Using examples, Dittrich and Thiemann [87] argue that the discreteness of the geometric operators, being gauge non-invariant, may not survive implementation in the full dynamics of LQG. Ceding the point in general, Rovelli [225] argues in favor of the reasonableness of physical geometric discreteness, showing in one case that the preservation of discreteness in the generally covariant context is immediate. In phenomenology this discreteness has been a source of inspiration for models. Nonetheless as the discussion of these operators makes clear, there are subtleties that wait to be resolved, either through further completion of the theory or, perhaps, through observational constraints on phenomenological models.

\subsection{Local Lorentz invariance and LQG}

It may seem that discreteness immediately gives rise to compatibility problems with LLI. For instance, the length derived from the minimum area eigenvalue may appear to be a new fundamental length. However, as SR does not contain an invariant length, must such a theory with a distinguished characteristic length be in contradiction with SR? That this is not necessarily the case has been known since the 1947 work of Snyder [250] (see [167] for a recent review).

In [230] Rovelli and Speziale explain that a discrete spectrum of the area operator with a minimal non-vanishing eigenvalue can be compatible with the usual form of Lorentz symmetry. To show this, it is not sufficient to set discrete eigenvalues in relation to Lorentz transformations, rather, one must consider what an observer is able to measure. The main argument of [230] is that in quantum theory the spectra of geometric variables are observer invariant, but expectation values are not. The authors explain this idea by means of the area of a surface. Assume an observer $\mathcal{O}$ measures the area of a small two-dimensional surface to be $A$, a second observer $\mathcal{O}^{\prime}$, who moves at a velocity $v$ tangential to the surface, measures $A^{\prime}$. In classical SR, when $\mathcal{O}$ is at rest with respect to the surface in flat space, the two areas are related as $A^{\prime}=\sqrt{1-v^{2}} A$. If $A$ is sufficiently small, this holds also in GR.

However this relation, which allows for arbitrarily small values of $A^{\prime}$, cannot be simply taken over as a relation between the area operators $\hat{A}$ and $\hat{A}^{\prime}$ in LQG. The above form suggests that $A^{\prime}$ is a simple function of $A$ and so $\hat{A}$ and $\hat{A}^{\prime}$ should commute. This is not the case. The velocity $v$, as the physical relative velocity between $\mathcal{O}$ and $\mathcal{O}^{\prime}$, depends on the metric, which of course is an operator, too. Rovelli and Speziale show that $\hat{v}$ does not commute with $\hat{A}$, and so $\left[\hat{A}, \hat{A}^{\prime}\right] \neq 0$. This means that the measurements of the area and the velocity of a surface are incompatible.

The apparent conflict between discreteness and Lorentz contraction is resolved in the following way: The velocity of an observer, who measures the area of a surface sharply, is completely undetermined with respect to this surface and has vanishing expectation value. The indeterminacy of the velocity means that an observer who measures the area $A$ precisely cannot be at rest with respect to the surface. On the other hand, an observer with a nonzero expectation value of velocity relative to the surface cannot measure the area exactly. For this observer the expectation value is Lorentz-contracted, whereas the spectrum of the area operator is the same. 
More recent considerations in the spin-foam framework can be found in [229], where the Hilbert space of functions on $\mathrm{SU}(2)$ is mapped to a set $\mathcal{K}$ of functions on $\mathrm{SL}(2, \mathbb{C})$ by the Dupuis-Livine map [89]. In this way $\mathrm{SU}(2) \mathrm{SNW}$ functions are mapped to $\mathrm{SL}(2, \mathbb{C})$ functions that are manifestly Lorentz covariant. Furthermore these functions are completely determined by their projections on $\mathrm{SU}(2)$, so $\mathcal{K}$ is linearly isomorphic to a space of functions on $\mathrm{SU}(2)$. It is shown in [229] that the transition amplitudes are invariant under $\operatorname{SL}(2, \mathbb{C})$ gauge transformations in the bulk and manifestly satisfy LLI.

While these papers suggest strongly that LLI is part of LQG - just as might be expected from a quantization of GR - other researchers have explored the possibility that the discreteness spoils or deforms LLI through the modification of dispersion relations and interaction terms.

From a fundamental theory point of view, the symmetry group associated to the field theory of the continuum approximation, from which particles acquire their properties through irreducible representations, will be dynamically determined by quantum gravity theory and the associated ground state. Originating in work by Kodama, a line work work contains hints that this group may be deformed.

Found in the late 80 's $[147,148,247]$, the Kodama state,

$$
\Psi_{\mathrm{K}}[A]=e^{\frac{3}{2 \Lambda G \hbar} S_{\mathrm{CS}}[A]},
$$

was a source of hope that one could model the ground state (and maybe excited states) of QG with a cosmological constant $\Lambda$. The phase, $S_{\mathrm{CS}}[A]$, is the Chern-Simons action for complex Ashtekar connections, with the same symmetry group as deSitter or anti deSitter space, according to the sign of $\Lambda$. The wavefunction $\Psi_{\mathrm{K}}$ is (locally) gauge invariant, spatially diffeomorphism invariant, and a solution to the Hamiltonian constraint of LQG for a (triads-on-left) factor ordering in complex Ashtekar variables. When $\Psi_{\mathrm{K}}[A]$ is multiplied by SNW functions of the connections a picture emerges in which states of quantum gravity are labeled by knot (or more accurately, graph) classes of framed spin networks $[188,192,247]$. The space-time has DeSitter as a semiclassical limit [247]. There is also an intriguing link between the cosmological constant and particle statistics [188].

It is well-known that for space-time with boundary, boundary terms and/or conditions must be added to the Einstein-Hilbert action to ensure that the variational principle is well defined and Einstein's equations are recovered in the bulk. (Possible boundary conditions and boundary terms for real Ashtekar variables were worked out in [137,188].)

However, there are severe difficulties with this choice of complex-valued self-dual connection variables and the Kodama state: The kinematic state space of complex-valued connections is not yet rigorously constructed - we lack a uniform measure. The state itself is both not normalizable in the linearized theory, violates CPT and is not invariant under finite gauge transformations (see [257] for discussion). An analysis of perturbations around the Kodama state shows that the perturbations of the Kodama state mix positive-frequency right-handed gravitons with negativefrequency left-handed gravitons [178]. The graph transform of the Kodama states, defined through variational methods, acquires a sensitivity to tangent space structure at vertices [185]. Finally, the original $q$-deformation of the loop algebra suggested in $[188,192]$ is inconsistent [186]. These difficulties have made further progress in this area challenging, although there is work on generalizing the Kodama state to real Ashtekar variables, where some of these issues are addressed [218].

Following the lead of developments in 3D gravity coupled to point particles, where particle kinematics is deformed when the topological degrees of freedom are integrated out, one may wonder whether a similar situation holds in $3+1$ when the local gravitational effects are integrated out [156]. In [156] the authors showed that, for BF theory with a symmetry breaking term controlled by a parameter [99,249,251], (point) particles enjoy the usual dispersion relations and any deformation appears only in interaction terms. 
In the next section we review frameworks in which the symmetry groups are deformed or broken.

\section{Quantum particle frameworks}

\subsection{Relativistic particles and plane-waves}

We start by recalling the fundamental structures associated with the physics of free particles in the phase space picture. Constructing a phenomenological model to incorporate the Planck scale consists in generalizing or modifying this structure.

A relativistic particle (with no spin) propagating in Minkowski spacetime is described in the Hamiltonian formalism by the following structures.

- A phase space $\mathcal{P} \sim T^{*} \mathbb{R}^{4} \sim \mathbb{R}^{4} \times \mathbb{R}^{4}$, the cotangent bundle of the flat manifold $\mathbb{R}^{4}$. It is parameterized by the configuration coordinates $x^{\mu} \in \mathbb{R}^{4}$ and the momentum coordinates $p_{\mu} \in \mathbb{R}^{4}$. These coordinates have a physical meaning, i.e. they are associated with outcome of measurements (e.g. using rods, clocks, calorimeters, etc.). $\mathcal{P}$ is equipped with a Poisson bracket, that is, the algebra of (differentiable) functions over the phase space $\mathcal{C}(\mathcal{P})$ is equipped with a map $\{\}:, \mathcal{C}(\mathcal{P}) \times \mathcal{C}(\mathcal{P}) \rightarrow \mathcal{C}(\mathcal{P})$ which satisfies the Jacobi identity. For the coordinate functions, the standard Poisson bracket is given by

$$
\left\{x^{\mu}, x^{\nu}\right\}=0, \quad\left\{x^{\mu}, p_{\nu}\right\}=\delta_{\nu}^{\mu}, \quad\left\{p_{\mu}, p_{\nu}\right\}=0
$$

- Symmetries given by the Poincaré group $\mathcal{P} \sim \mathrm{SO}(3,1) \ltimes \mathcal{T}$, given in terms of the semidirect product of the Lorentz group $\mathrm{SO}(3,1)$ and the translation group $\mathcal{T}$. So that there exists an action of the Lorentz group on the translation, which we note $\Lambda \triangleright h, \forall(\Lambda, h) \in \mathcal{P}$. The product of group elements is hence given by

$$
\left(\Lambda_{1}, h_{1}\right)\left(\Lambda_{2}, h_{2}\right)=\left(\Lambda_{1} \Lambda_{2}, h_{1}\left(\Lambda_{1} \triangleright h_{2}\right)\right) .
$$

The Lie algebra $\mathfrak{P}$ of $\mathcal{P}$ is generated by the infinitesimal Lorentz transformations $J_{\mu \nu}$ and translations $T_{\mu}$ which satisfy

$$
\begin{aligned}
& {\left[J_{\mu \nu}, J_{\alpha \beta}\right]=\eta_{\mu \beta} J_{\nu \alpha}+\eta_{\nu \alpha} J_{\mu \beta}-\eta_{\mu \alpha} J_{\nu \beta}-\eta_{\nu \beta} J_{\mu \alpha}} \\
& {\left[T_{\alpha}, T_{\beta}\right]=0, \quad\left[J_{\mu \nu}, T_{\alpha}\right]=\eta_{\nu \alpha} T_{\mu}-\eta_{\mu \alpha} T_{\nu} .}
\end{aligned}
$$

The action of $\mathfrak{P}$ is given on the phase space coordinates by

$$
\begin{aligned}
& J_{\mu \nu} \triangleright x^{\alpha}=\eta_{\nu}^{\alpha} x_{\mu}-\eta_{\mu}^{\alpha} x_{\nu}, \quad J_{\mu \nu} \triangleright p_{\alpha}=\eta_{\nu \alpha} p_{\mu}-\eta_{\mu \alpha} p_{\nu}, \\
& T_{\mu} \triangleright x^{\nu}=\delta_{\mu}^{\nu}, \quad T_{\mu} \triangleright p_{\nu}=0 .
\end{aligned}
$$

This is extended naturally to the functions on phase space.

- Particle dynamics given by the mass-shell or dispersion relation ${ }^{1} p^{2}=p_{\mu} \eta^{\mu \nu} p_{\nu}=m^{2}$. This is a constraint on phase space which implements the time reparameterization invariance of the following action

$$
B=\int \mathrm{d} \tau\left(\dot{x}^{\mu} p_{\mu}-\lambda\left(p^{2}-m^{2}\right)\right) .
$$

$\lambda$ is the Lagrange multiplier implementing the constraint $p^{2}-m^{2}=0$. This action contains the information about the phase space structure and the dynamics. We can perform

\footnotetext{
${ }^{1}$ We use the metric $\eta^{\mu \nu}=\operatorname{diag}(+,-,-,-)$.
} 
a Legendre transform in the massive case (or a Gauss transform in the massless case) to express this action in the tangent bundle $T \mathbb{R}^{4}$,

$$
ß=m \int \mathrm{d} \tau \sqrt{\dot{x}^{\mu} \dot{x}^{\nu} \eta_{\mu \nu}(x)}, \quad \dot{x}^{\mu}=\frac{d x^{\mu}}{d \tau} .
$$

With this description, we recover the familiar fact that the relativistic particle worldline given by a geodesic of the metric.

When we require the Poincaré symmetries to be consistent with all these phase space and particle dynamics structures, these pieces fit together very tightly.

- The Poincaré symmetries should be compatible with the Poisson bracket. If we define our theory in a given inertial frame, physics will not change if we use a different inertial frame, related to the initial one by a Poincaré transformation $t$,

$$
\begin{aligned}
& \left\{f_{1}(x, p), f_{2}(x, p)\right\}=f_{3}(x, p) \\
& \quad \Leftrightarrow \quad\left\{f_{1}(t \triangleright x, t \triangleright p), f_{2}(t \triangleright x, t \triangleright p)\right\}=f_{3}(t \triangleright x, t \triangleright p), \quad f_{i} \in \mathcal{C}(P) .
\end{aligned}
$$

- The mass-shell condition/dispersion relation $p^{2}=m^{2}$ encodes the mass Casimir of the Poincaré group. As such this mass-shell condition is invariant under Lorentz transformations.

When dealing with fields or multi-particles states, we have also the following important structures.

- The total momentum of many particles is obtained using a group law for the momentum, adding extra structure to the phase space. We are using $\mathbb{R}^{4}$, which is naturally equipped with an Abelian group structure ${ }^{2}$. From this perspective, one can consider the phase space as a cotangent bundle over the group $\mathbb{R}^{4}$. This picture will be at the root at the generalization to the non-commutative case.

- Plane-waves $e^{i x^{\mu} k_{\mu}}$, where $k_{\mu}$ is the wave-covector, are an important ingredient when we deal with field theories. The plane-wave is usually seen as the eigenfunction of the differential operators encoding the infinitesimal translations on momentum or configuration space

$$
\partial_{x^{\mu}} e^{i x^{\nu} k_{\nu}}=i k_{\mu} e^{i x^{\nu} k_{\nu}}, \quad \partial_{k_{\mu}} e^{i x^{\nu} k_{\nu}}=i x^{\mu} e^{i x^{\nu} k_{\nu}} .
$$

Since the momentum operator $P_{\mu}$ is usually represented as $-i \partial_{x^{\mu}}$, it is natural to identify the wave-covector to the momentum $k_{\mu}=p_{\mu}$. When this identification is implemented, the product of plane-waves is intimately related to the addition of momenta, hence the group structure of momentum space.

$$
e^{i x \cdot p_{1}} e^{i x \cdot p_{2}}=e^{i x \cdot\left(p_{1}+p_{2}\right)} .
$$

- The infinitesimal translation $T_{\mu}$ is represented as $\partial_{x^{\mu}}$ therefore it can be related to the momentum operator from (3.1). Modifying momentum space is then synonymous to modifying the translations.

As we are going to see in the next sections, introducing QG effects in an effective framework will consist in modifying some of the above structures, either by brute force by breaking some symmetries or, in a smoother way, by deforming these symmetries.

\footnotetext{
${ }^{2} \mathbb{R}^{4}$ is even a vector space but for the generalizations we shall consider, it is only the group structure that is relevant.
} 


\subsection{Introducing Planck scales into the game: modified dispersion relations}

Light, or the electromagnetic field, is a key object to explore the structure of spacetime. In 1905, light performed a preferred role in understanding Special Relativity. In 1919, Eddington measured the bending of light induced by the curvature of spacetime. As a consequence, these results pointed to the fact that a Lorentzian metric is the right structure to describe a classical spacetime.

In the same spirit, a common idea behind QG phenomenology is that a semi-classical spacetime should leave imprints on the propagation of the electromagnetic field such as in [21] discussed in the Introduction. In this example the lever arm that raises possible QG effects into view is the proposed cumulative effects over great distances.

The concept of a modified dispersion relation (MDR) is at the root of most QG phenomenology effective theories. Depending on the approach one follows, there can also be some modifications at the level of the multiparticle states, i.e. how momenta are added. One can readily see that such a modified dispersion relation is not consistent with the Lorentz symmetries, so that they have to be broken or deformed. We shall discuss both possibilities below.

There is nevertheless a semi-classical regime where the Planck scale is relevant and possible non trivial effects regarding symmetries could appear. Indeed, the natural flat semi-classical limit in the QG regime is given by $^{3} \Lambda, G, \hbar \rightarrow 0$. There are a number of possibilities to implement these limits [97]. An interesting flat semi-classical limit is when $\Lambda=0$ and $\frac{\hbar}{G}=\kappa^{2}$ is kept constant in the limit $G, \hbar \rightarrow 0$. This regime is therefore characterized by a new constant $\kappa$, which has dimension either energy, momentum or mass. Note that in this regime the Planck length $\ell_{\mathrm{P}}^{2}=\hbar G$ naturally goes to zero, hence there is no minimum length from a dimensional argument. The key question is how to implement this momentum scale $\kappa$, that is to identify the physical motivations which will dictate how to encode this scale in the theory.

Following the paper by Amelino-Camelia et al. [21], many modeled potentially observable QG effects with "semi-classical" effective theories. In some cases discreteness was put in "by hand". In others deviations from Special Relativity, suppressed by the ratio (particle energy)/(QG scale) or some power of it, were modeled. This is the approach followed when considering Lorentz symmetry violation discussed in Section 4.1. Another approach taken was to introduce the Planck length in the game as a minimum length and investigate possible consequences. For a recent review on this notion and implications of minimum length see [134]. Alternatively the Planck energy, or the Planck momentum, was set as the maximum energy [69] (or maximum momentum) that a fundamental particle could obtain. Implementing this feature can also generate a modified dispersion relation. This is the approach which is often considered in the deformed symmetries approach.

Both of these later proposals affect dispersion relations and hence the Poincaré symmetries. Therefore in the regime $\lim _{\hbar, G \rightarrow 0} \frac{\hbar}{G}=\kappa^{2}$, it is not clear that the symmetries must be preserved and some non-trivial effects can appear.

In general, the idea is to cook up more or less rigorously an effective model and then try to relate it to a given QG model (bottom-top approach). The models which are (the most) well defined mathematically are, to our knowledge, given by the non-commutative approach and the Finsler geometry approach. Among these two, Finsler geometry is the easiest to make sense at the physical level.

There are fewer attempts to derive semi-classical effects from QG models (top-down approach). Most of the time, these attempts to relate the deep QG regime and the semi-classical are heuristic: there is no real complete QG theory at this time and the semi-classical limit is often problematic. We shall review some of them when presenting the different QG phenomenological

\footnotetext{
${ }^{3} \Lambda$ is the cosmological constant, $G$ the Newton constant and $\hbar$ the Planck constant.
} 
models. Even though these attempts were few and heuristic, they were influential, promoting the idea that it is possible to measure effects originating at the Planck scale.

Currently, QG phenomenology is therefore not firmly tied to a particular quantum theory of gravity. For a brief, general review over quantum gravity phenomenology, independent of a fundamental theory, see [164]. Contemporary observational data are not sufficient to rule out QG theories, not only because of the lack of stringent data, but particularly because the link between fundamental theories and QG phenomenology is loose. Nevertheless, present observational data restrict parameters in some models, effectively ruling out certain modifications, such as cubic modifications to dispersion relations in the effective field theory (EFT) context. We shall review this in Section 4.1.

In the following we are going to present the main candidates to encode some QG effective semi-classical effects. When available we shall also recall the arguments relating them to LQG. As a starter, we now recall different arguments which attempt to justify a MDR from the LQG perspective.

\subsection{Arguments linking modified dispersion relations and LQG}

We present three quite different strategies to establish a firmer tie between LQG and modified dispersion relations. The first one introduces a heuristic set of weave states, flat and continuous above a characteristic scale $L$, and then expands the fields around this scale. The second strategy starts from full LQG and aims at constructing quantum field theory (QFT) on curved spacetime which is an adaptation of conventional QFT to a regime of non-negligible, but not too strong gravitational field. In this construction coherent states of LQG are employed, which are quantum counterparts of classical flat space. Due to the enormous complications, this venture must resort to many approximations. The third strategy deals in a very general way with quantum fluctuations around classical solutions of GR. This approach is rather sketchy and less worked-out in details. Given the preliminary stage of development of LQG all of the derivations employ additional assumptions. Nevertheless they provide a starting point for exploring the possible effects of the discreteness of LQG.

Departures from the standard quadratic energy-momentum relations and from the standard form of Lorentz transformations can of course originate from the existence of a preferred reference frame in the limit of a vanishing gravitational field, i.e. a breaking of Lorentz invariance at high energies. Nevertheless, this need not necessarily be the case. The relativity principle can be valid also under the conditions of modified dispersion relations and Lorentz transformations. In [19] the compatibility of a second invariant quantity in addition to the speed of light, a length of the order of the Planck length, with the relativity principle was shown. The product of this length with a particle energy is a measure for the modification of the dispersion relation. Frameworks with two invariant scales, where the second one may also be an energy or a momentum, were dubbed "doubly special relativity theories" (DSR). As an outcome of the theory's development, it was found that "DSR" may also be an acronym for "deformed special relativity" in that Poincaré Lie algebra of symmetry generators, namely the energy and momentum operators, may be deformed or embedded into a Hopf algebra [182], whereas in the doubly special relativity framework the representation of the Poincaré group, i.e. the action on space-time or momentum space, is nonlinearly deformed. Deformed algebras are used in the $\kappa$-Minkowski and in the $\kappa$-Poincaré approach [154,170]. For relations to doubly special relativity see [155].

\subsubsection{MDR from weave states}

Following the first strategy of introducing an heuristic state Gambini and Pullin [101] modeled a low energy semi-classical kinematic state with a "weave", a discrete approximation of smooth flat geometry, characterized by a scale $L$. In an inertial frame, the spatial geometry reveals its 
atomic nature below the characteristic length scale. Above this length scale $L$, space appears flat and continuous. In this preferred frame the expectation value of the metric is of the form

$$
\left\langle q_{a b}\right\rangle=\delta_{a b}+O\left(\frac{\ell_{\mathrm{P}}}{L}\right) .
$$

To see the leading order effect for photons Gambini and Pullin analyzed the Maxwell Hamiltonian,

$$
H=\frac{1}{2} \int \mathrm{d}^{3} x \frac{q_{a b}}{\sqrt{q}}\left(E^{a} E^{b}+B^{a} B^{b}\right)
$$

importing one key idea from LQG. The densitized metric operator $q_{a b} / \sqrt{q}$ is expressed as a product of two operators $\hat{w}_{a}\left(v_{i}\right)$, which are commutators of the connection and the volume operator. These operators are finite and take non-vanishing values only at vertices $v_{i}$ of the graph. Regulating the Hamiltonian with point splitting, the authors took the expectation value of the Hamiltonian in the weave state, averaging over a cell of size $L$. They expanded the fields around the center of the cell $P$ and found that the leading order term

$$
\left\langle\hat{w}_{a}\left(v_{i}\right) \hat{w}_{b}\left(v_{j}\right)\right\rangle\left(v_{i}-P\right)_{c}
$$

is a tensor with three indices. Assuming rotational symmetry, this term is proportional to $\epsilon_{a b c} \ell_{\mathrm{P}} / L$, thus modifing Maxwell's equations. The correction is parity violating. The resulting dispersion relations enjoy cubic modifications, taking the form

$$
\omega_{ \pm}^{2}=k^{2} \mp 4 \chi \frac{k^{3}}{\kappa}
$$

in the helicity basis. The constant $\chi$ was assumed to be order 1 . Hence the weave states led to birefringence. As discussed in Section 4.1.2 these effects may be constrained by observation. Furthermore some theoretical arguments can also be proposed against the validity of such proposal as we shall see in Section 3.4.

Taking a similar approach and specifying general properties of a semi-classical state, Alfaro et al. found that, in an analysis of particle propagation, photon [12] and fermion [11,13] dispersion relations are modified. They find these by applying LQG techniques on the appropriate quantum Hamiltonian acting on their states. Following similar steps to Gambini and Pullin, Alfaro et al. expand the expectation value of the matter Hamiltonian operators in these states.

To determine the action of the Hamiltonian operator of the field on quantum geometry Alfaro et al. specify general conditions for the semi-classical state. The idea is to work with a class of states for geometry and matter that satisfy the following conditions:

1. The state is "peaked" on flat and continuous geometry when probed on length scales larger than a characteristic scale $L, L \gg \ell_{\mathrm{P}}$.

2. On length scales larger than the characteristic length the state is "peaked" on the classical field.

3. The expectation values of operators are assumed to be well-defined and geometric corrections to the expectations values may be expanded in powers of the ratio of the physical length scales, $L$ and $\ell_{\mathrm{P}}$.

The authors dub these states "would-be semi-classical states". States peaked on flat geometry and a flat connection are expected for semiclassical or coherent states that model flat space. Lacking the quantum Hamiltonian constraint for the gravitational field and thus also for the associated semi-classical states, the work of Alfaro et al. is necessarily only a forerunner of the 
detailed analysis of semi-classical states. See [233,234] for further work on semiclassical states and dispersion relations. To parameterize the scaling of the expectation value of the gravitational connection in the semiclassical state the authors introduce a parameter $\Upsilon$ that gives the scaling of the expectation value of the geometric connection in the semi-classical state $|W \phi\rangle$

$$
\left\langle W \phi\left|A_{a}^{i}\right| W \phi\right\rangle \sim \frac{1}{L}\left(\ell_{\mathrm{P}} L\right)^{\Upsilon} \delta_{a}^{i}
$$

where $\phi$ are the matter fields. The determination of the scaling is a bit of a mystery. Alfaro et al. propose two values for $L$ : The "mobile scale" where $L=1 / p$, and the "universal" value where $L$ is a fixed constant, $p$ is the magnitude of the 3-momentum of the particles under consideration. We will see in the next section that matching the modifications to the effective field theory suggests a universal value $L \gg \ell_{\mathrm{P}}$ and $\Upsilon \geq 0$, so we will use the universal value. It is not surprising that Lorentz-violating (LV) terms arise when the spatial distance $L$ is introduced.

Expanding the quantum Hamiltonian on the semi-classical states Alfaro et al. find that particle dispersion relations are modified. Retaining leading order terms in $p / \kappa$, the scaling with $(L \kappa)$ and next to leading order terms in $\kappa$, but dropping all dimension 3 and 4 modifications for the present, the modifications are, for fermions,

$$
E_{ \pm}^{2} \simeq\left[1+2 \kappa_{1}(L \kappa)^{-\Upsilon-1}\right] p^{2}+m^{2} \pm \frac{\kappa_{9} m^{2}}{\kappa} p \mp \frac{\kappa_{7}}{2 L \kappa^{2}}(L \kappa)^{-\Upsilon} p^{3}-\frac{\kappa_{3}}{\kappa^{2}} p^{4}
$$

where $p$ is the magnitude of the 3 -momentum and the dimensionless $\kappa_{i}$ parameters are expected to be $O(1)$ (and are unrelated to the Planck scale $\kappa$. The labels are for the two helicity eigenstates. These modifications are derived from equation (117) of [13], retaining the original notation, apart from the Planck mass $\kappa$.

Performing the same expansion for photons Alfaro et al. find that the semi-classical states lead to modifications of the dispersion relations, at leading order in $k / \kappa$ and scaling $(L \kappa)$

$$
\omega_{ \pm}^{2} \simeq k^{2}\left[1+2 \theta_{7}(L \kappa)^{-2-2 \Upsilon}\right] \pm \frac{4 \theta_{8}}{\kappa}\left[1+2 \theta_{7}(L \kappa)^{-2-2 \Upsilon}\right] k^{3}+\left(2 \theta_{8}-4 \theta_{3}\right) \frac{k^{4}}{\kappa^{2}}
$$

where the $\theta_{i}$ parameters are dimensionless and are expected to be $O(1)$. The leading order term is the same polarization-dependent modification as proposed in Gambini and Pullin [101]. In the more recent work [233,234] the structure of the modification of the dispersion relations was verified but, intriguingly, the corrections do not necessarily scale with an integer power of $\kappa$.

As is clear in the derivation these modified dispersion relations (MDR) manifestly break LLI and so are models of LQG with a preferred frame. The effects are suppressed by the Planck scale, so any $O(1)$ constraints on the parameters are limits placed on Planck-scale effects. These constraints, without a complete dynamical framework that establishes the conservation, or deformation, of energy and momentum, must come from purely kinematic tests.

Interestingly, as we will see in Section 4.1, Alfaro et al. found the modifications to the dispersion relations corresponding to the dimension 5 and the CPT-even dimension $6 \mathrm{LV}$ operators in the effective field theory framework. Of course given the limitations of the model they did not derive the complete particle dynamics of the EFT framework.

It was suggested in [157] that different choices for the canonical variables for the U(1) field theory could remove the Lorentz violating terms. However Alfaro et al. pointed out that this is inconsistent; the only allowed canonical pairs in LQG are those that have the correct semiclassical limit and are obtained by canonical transformation [10].

Finally, we must emphasize that these derivations depend critically on assumptions about the semi-classical weave state, the source of the local Lorentz symmetry violations. 


\subsubsection{Quantum field theory in curved space from LQG}

Sahlmann and Thiemann studied dispersion relations in a framework of QFT on curved space from basic LQG principles by heavily making use of approximations [233,234]. In the first step QFTs on discrete space are constructed on an essentially kinematic level. Rather than taking the total Hamiltonian constraint of gravity and matter, the matter Hamiltonians of gauge, bosonic and fermionic fields are treated as observables, dependent on geometric variables of the background. Then the gravitational field is assumed to be in a coherent state, where expectation values for field variables yield the classical values and the quantum uncertainties are minimal.

The Hilbert space of matter states $\mathcal{H}_{\mathrm{m}}^{\text {Fock }}(m)$ depends on the state $g$ of the geometry. The vacuum state $\Omega_{\mathrm{m}}(g)$ is the ground state of some (geometry-dependent) matter Hamiltonian operator $\hat{H}_{\mathrm{m}}(g)$. In QG $m$ becomes an operator, and so $\Omega_{\mathrm{m}}(g)$ becomes a "vacuum operator", i.e. a function of the matter degrees of freedom with values in $\mathcal{L}\left(\mathcal{H}_{\text {geom }}^{\text {kin }}\right) \otimes \mathcal{H}_{\mathrm{m}}^{\text {kin }} \cdot \mathcal{L}$ is the space of linear operators on a background independent Hilbert space of kinematic states of geometry, $\mathcal{H}_{\mathrm{m}}^{\text {kin }}$ is a kinematic matter Hilbert space. From this vacuum operator a vacuum state may be constructed in principle as expectation value in a state of quantum geometry, which is peaked at classical flat space.

As a technical detail and interesting twist, the construction of annihilation and creation operators involves fractional powers of the Laplacian on a background metric. In [233, 234] these operators are constructed but the spectra required to calculate fractional powers are not known. To circumvent this problem the expectation values of the Laplacian in a coherent state, mimicking flat space, are calculated first, then fractional powers are taken. In coherent states this approximation coincides with exact calculations in zeroth order in $\hbar$. The coherent state employed is modeled by spin networks with an irregular 6-valent lattice. Creation and annihilation operators, an approximate vacuum state, and approximate Fock states are constructed from the matter Hamiltonians in the sense of the described approximation. The influence of geometry is included in an effective matter Hamiltonian, the matrix elements of which are defined in the following way

$$
\left\langle\psi_{\mathrm{m}}, \hat{H}_{\mathrm{m}}^{\mathrm{eff}}(g) \psi_{\mathrm{m}}^{\prime}\right\rangle_{\mathcal{H}_{\mathrm{m}}^{\mathrm{kin}}}:=\left\langle\psi_{\mathrm{grav}}(g) \otimes \psi_{\mathrm{m}}, \hat{H} \psi_{\mathrm{grav}}(g) \otimes \psi_{\mathrm{m}}^{\prime}\right\rangle_{\mathcal{H}_{\mathrm{grav}}^{\mathrm{kin}} \otimes \mathcal{H}_{\mathrm{m}}^{\mathrm{kin}}} \cdot
$$

Here the general building principle of a matter-geometry Hamiltonian, related to a graph $\Gamma$ is

$$
\hat{H}_{\Gamma}=\sum_{v, l ; v^{\prime}, l^{\prime}} \hat{M}_{l}(v)^{\dagger} \hat{G}\left(v, l ; v^{\prime}, l^{\prime}\right) \hat{M}_{l^{\prime}}\left(v^{\prime}\right),
$$

where $\hat{M}$ a matter operator with some discrete (collective, matter and geometry) label $l, v$ is a SNW vertex and $\hat{G}$ is an operator of geometry.

Recently work in the context of cosmological models has also found hints of a modified dispersion relation [84]. Working in the context of a quantized Bianchi I model with a scalar field, the authors found, when taking back-reaction into account, that the scalar field modes propagate on a wave number-dependent metric and the dispersion relation is modified [84].

The discreteness and irregularity of the underlying lattice breaks translation and rotation symmetry. There are no exact plane wave solutions for matter fields, only in the long distance limit the irregularities average out and so for long wavelengths - compared to the lattice spacing - there are at least approximate plane waves. In this limit the matter Hamiltonians simplify sufficiently, so that the sketched program becomes feasible and yields an energy-momentum dispersion relation for low energies, which carries the imprints of both discreteness and fluctuations.

With these possible modifications to particle dispersion relations, the obvious next step is to explore the effects that arise from these Lorentz violating modifications. Early studies $[140,149]$ used particle kinematics phenomenology, modified dispersion relations plus energymomentum conservation. We now know, see e.g. [141], that constraints require a full dynamical 
framework for the fields. The most obvious, and certainly most developed framework is effective field theory. We review the physical effects of cubic modifications to the dispersion relations in effective field theory in Section 4.1. We briefly discuss alternate frameworks and higher dimension modifications in Sections 3.10 and 4.1.7.

\subsubsection{MDR from Hamilton-Jacobi theory}

In [245] by Smolin the occurrence of corrections to particle kinematics in the low-energy limit of $\mathrm{QG}$ is made plausible in a very general way. This derivation is based on the quantum fluctuations around classical GR in the connection representation. The dynamics is formulated in the Hamilton-Jacobi theory with the aid of the action functional $S[A]$. Canonical conjugate momenta are given by

$$
E^{a}{ }_{i}(x)=\frac{1}{\rho} \frac{\delta S[A]}{\delta A_{a}{ }^{i}(x)},
$$

with $\rho$ being a constant with dimension (length) ${ }^{2}$. The solutions to the dynamics form a trajectory $\left(A^{0}{ }_{a}{ }^{i}(t), E^{0}{ }_{i}(t)\right)$ with some parameter $t$. The parameter can be chosen to be proportional to the Hamilton-Jacobi functional. This functional, in turn, can be written as an integral over a density $\mathcal{S}$ on the spatial manifold, or some local coordinate neighborhood,

$$
S[A]=\int_{\Sigma} \mathcal{S}[A]
$$

Therefore, on the classical space-time there exists a time $T$ proportional to $\mathcal{S}[A]$, defining a slicing. The slicing constructed in this way is determined by the classical solution. When connections depart slightly from the classical trajectory the slicing fluctuates. So variations of functions on configuration space, evaluated at the classical trajectory, can be related to variations on space-time, expressed as

$$
\frac{\mathrm{d}}{\mathrm{d} T}=\mu \frac{\delta}{\delta \mathcal{S}[A]}
$$

where $\mu$ is a constant of dimension (length) ${ }^{-1}$. The solution $E^{0^{a}}{ }_{i}(t)$ defines a matrix

$$
g=-\mathrm{d} T^{2}+\sum_{i} e_{i}^{0} \otimes e_{i}^{0}
$$

with the orthonormal inverse triad $e_{i}^{0}$ related to $E^{0}{ }_{i}$.

In the neighbourhood of a classical trajectory the connection $A$ can be formulated through a dependence on $\mathcal{S}$ and quantities $a_{a}{ }^{i}$ [248], so that

$$
\frac{\delta}{\delta A_{a}{ }^{i}(x)}=\frac{1}{M} E^{0}{ }_{i} \frac{\delta}{\delta \mathcal{S}}+\frac{\delta}{\delta a_{a}{ }^{i}}
$$

The first term can be understood as variation in the internal time coordinate, $a_{a}{ }^{i}$ contains the gravitational degrees of freedom.

Going over to quantum theory we construct the operator

$$
\hat{E}^{a}{ }_{i}(x)=-\hbar \rho \frac{\delta}{\delta A_{a}^{i}(x)}
$$

and the semiclassical state functional

$$
\Psi_{0}[A]=e^{i \frac{S[A]}{\hbar}} \quad \text { with } \quad \hat{E}^{a}{ }_{i} \Psi_{0}[A]=E^{0^{a}}{ }_{i} \Psi_{0}[A] .
$$


To study semiclassical QG effects on the propagation of a matter field $\phi$, Smolin takes quantum states in the Born-Oppenheimer form $\Psi[A, \phi]=\Psi_{0}[A] \chi[A, \phi]$. In the neighborhood of the classical trajectory

$$
\chi[A, \phi]=\chi\left[\mathcal{S}, a_{a}^{i}, \phi\right]
$$

and the action of $\hat{E}^{a}$ on such functions is

$$
{\hat{E^{a}}}_{i}(x) \chi[A, \phi]=-i \hbar \rho \frac{\delta \chi[A, \phi]}{\delta A_{a}{ }^{i}(x)}=\left(E^{0^{a}}{ }_{i} \frac{i \hbar \rho}{M} \frac{\delta}{\delta \mathcal{S}(x)}-i \hbar \rho \frac{\delta}{\delta a_{a}{ }^{i}(x)}\right) \chi\left[\mathcal{S}, a_{a}{ }^{i}, \phi\right] .
$$

By (3.4) we have

$$
\frac{i \hbar \rho}{M} \frac{\delta}{\delta \mathcal{S}(x)}=\frac{i \hbar \rho}{M \mu} \frac{\mathrm{d}}{\mathrm{d} T},
$$

where $\frac{\hbar \rho}{M \mu}$ has dimension of length or time in natural units. The only length scale in the problem (leaving aside $\Lambda$ ) being the Planck length, we may write

$$
\frac{\hbar \rho}{M \mu}=\alpha \ell_{\mathrm{P}} \equiv \frac{\alpha}{\kappa}
$$

where the constant $\alpha$ is determined later on in [245].

On the classical trajectory $\chi\left[\mathcal{S}, a_{a}{ }^{i}, \phi\right]=\chi\left[T, a_{a}{ }^{i}, \phi\right]$. At the semiclassical level we can neglect $\delta / \delta a_{a}{ }^{i}$, which describes couplings of matter to gravitons. Finally we have

$$
\hat{E}^{a}{ }_{i} \Psi[A, \phi]=\Psi_{0}[A] E^{0^{a}}{ }_{i}\left(1-i \alpha \ell_{\mathrm{P}} \frac{\mathrm{d}}{\mathrm{d} T}\right) \chi\left[T, a_{a}{ }^{i}, \phi\right] .
$$

Now consider a semiclassical state of definite frequency with respect to the time $T$,

$$
\chi\left[T, a_{a}{ }^{i}, \phi\right]=e^{-i \omega T} \chi_{\omega}\left[a_{a}{ }^{i}, \phi\right] .
$$

The action of the triad operator on such a function is

$$
\hat{E}^{a}{ }_{i}(x) \Psi[A, \phi]=\Psi_{0}[A] E^{0^{a}}{ }_{i}\left(1-\alpha \ell_{\mathrm{P}} \omega\right) \chi_{\omega}\left[a_{a}{ }^{i}, \phi\right] .
$$

So the classical solution $E^{0^{a}}{ }_{i}(x, T)$ effectively goes over into

$$
E^{0^{a}}{ }_{i}(x, T, \omega)=E^{0^{a}}{ }_{i}(x, T)\left(1-\alpha \ell_{\mathrm{P}} \omega\right),
$$

which implies an energy-dependent spatial metric,

$$
g \rightarrow g(\omega)=-\mathrm{d} T \otimes \mathrm{d} T+\sum_{i} e_{i}^{0} \otimes e_{i}^{0}\left(1-\alpha \ell_{\mathrm{P}} \omega\right)
$$

This may be interpreted as a "rainbow metric" [180]. Assuming that the corresponding contravariant metric in momentum space to be the inverse spatial metric, we arrive at a universal modification of dispersion relations,

$$
m^{2}=-g(\omega)^{\mu \nu} k_{\mu} k_{\nu}=\omega^{2}-\frac{k_{i}^{2}}{1-\alpha \ell_{\mathrm{P}} \omega} .
$$

In view of the universality of the effect - the independence of the form of matter in discussion and the absence of any (explicit) preferred frame vector field, which could distinguish a preferred reference frame, it is argued in [245] that the proper framework for these MDR is not Lorentz invariance breaking but a deformation with a helicity-independent, energy-dependent speed of photons. We shall come back to this point in Section 3.7.

In [244] a more general formulation is presented, which does not rely on connection representation and so is not restricted to LQG, but rather makes Lorentz invariance deformation plausible for a wider class of QG theories. This attribute is shared with the models described in the next section. 


\subsection{Broken Poincaré symmetries: Finsler geometry}

As we recalled in Section 3.2, a common idea behind QG phenomenology is that a semi-classical spacetime should leave some imprint on the propagation of light, in particular through a modified dispersion relation. A natural way to encode this idea is to approximate a semi-classical spacetime by a medium whose properties are to be determined by the specific underlying QG theory. As is known in solid state physics, the description of light propagation in a special medium (see, for example, references in [242]) is conveniently expressed with Finsler metrics, which are a generalization of the notion of Lorentzian/Riemannian metrics. From this perspective, it seems then quite natural to explore Finsler geometries as a candidate to describe effectively QG semi-classical effects [109]. The mathematics behind Finsler geometries are not yet as solid as in the Riemannian or Lorentzian geometry cases. For example the notions of signature and curvature of a Finsler metric are still active topics of discussion among the specialists. Finsler geometry provides however a nice framework to develop new mathematics and QG phenomenology. In fact it also provides ways to theoretically constrain the possible QG phenomenological proposals $[219,238]$.

Let us recall the construction. The propagation of the electromagnetic field $u^{A}=\left(E^{i}, B^{j}\right)$ (Capital Latin letters designate a pair of spatial indices.), in this medium/semi-classical spacetime is described by some effective Maxwell equations. We assume here that for simplicity, spacetime is $\mathbb{R}^{4}$. Following [219], we will make the assumption that these effective equations are still linear partial differential equations

$$
D_{A B}^{\kappa} u^{B}(x)=\sum_{n=1}^{N} Q_{A B}^{\alpha_{1} \cdots \alpha_{n}}(\kappa) \partial_{\alpha_{1}} \cdots \partial_{\alpha_{n}} u^{B}(x)=0 .
$$

The spacetime indices $\alpha_{i}$ run from 0 to 3 . We note the presence of the scale $\kappa$ which encodes the QG effects. The standard Maxwell equations are recovered when $\kappa \rightarrow 0$

$$
D_{A B}^{\kappa=0} u^{B}=\left(\begin{array}{cc}
-\delta_{i k} \partial_{0} & \epsilon_{i j l} \partial_{j} \\
\epsilon_{i j k} \partial_{j} & \delta_{i l} \partial_{0}
\end{array}\right)\left(\begin{array}{c}
E_{k} \\
B_{l}
\end{array}\right)=0 \Leftrightarrow\left\{\begin{array}{l}
-\partial_{0} E_{i}+\epsilon_{i j l} \partial_{j} B_{l}=0 \\
\epsilon_{i j k} \partial_{j} E_{k}+\partial_{0} B_{i}=0 .
\end{array}\right.
$$

Assuming there are no non-linear effects means that the coefficients $Q_{A B}^{\alpha_{1} \cdots \alpha_{n}}(\kappa)$ do not depend on the fields $u^{A}$ (but they could depend on the position $x$ ). This is not such a strong restriction since the existing proposals of QG modified Maxwell equations such as Gambini-Pullin's [101] are of this type (see Section 3.3). To solve these equations, it is common to use the short wave-length approximation, i.e. the Eikonal approximation. This means that we consider the particle approximation of the electromagnetic field. Skipping the details found in [219], this approximation leads to the modified dispersion relations or mass-shell constraints in terms of momentum $p$

$$
\mathcal{M}(x, \kappa, p)=\operatorname{det}\left(Q^{\alpha_{1} \cdots \alpha_{n}}(\kappa) p_{\alpha_{1}} \cdots p_{\alpha_{n}}\right)=0 .
$$

This is the eikonal equation, which can be seen as the covariant dispersion relation. The reader can quickly check that keeping only the usual Maxwell equation, leads to a standard dispersion relation $^{4} \mathcal{M}(x, \kappa=0, p)=\eta^{\mu \nu} p_{\mu} p_{\nu}=p^{2}=0$. The particle approximation gives rise to an action, expressed in phase space or the cotangent space $T^{*} \mathbb{R}^{4}$, of the type

$$
\beta_{\text {mod }}=\int \mathrm{d} \tau\left(\dot{x}^{\mu} p_{\mu}-\lambda(\mathcal{M}(x, \kappa, p))\right) .
$$

\footnotetext{
${ }^{4} \eta^{\mu \nu}$ is the Minkowski metric expressed in the cotangent bundle.
} 
If one wants to introduce the concept of energy $E$ and spatial momentum $\vec{p}$, we need to introduce an observer frame ${ }^{5}$. However, we need the frame $e$ expressed in the cotangent bundle. We recall that the Gauss map $G: T^{*} \mathbb{R}^{4} \rightarrow T \mathbb{R}^{4}$ in the massless case (or the Legendre transform in the massive case) allows to jump from a description in the cotangent bundle to the tangent bundle. Indeed, from the variations of the action, we obtain $\dot{x}$ in terms of $p$. The Gauss map (and the Legendre transform) inverts this expression. Explicitly, this map allows to express momentum (i.e. a covector) as a function of vectors ${ }^{6}$

$$
\dot{x}^{\mu}=\lambda \frac{\partial \mathcal{M}(x, \kappa, p)}{\partial p_{\mu}} \longrightarrow p_{\mu}=f_{\kappa}\left(x, \dot{x}^{\mu}, \lambda\right) .
$$

Let us postpone to later the discussion on the existence of such a map and let us assume it exists and it is invertible. The observer is encoded by a curve in the spacetime manifold $\mathbb{R}^{4}$ and its tangent vector $v^{\mu}$ defines the time part $\epsilon_{0}{ }^{\mu}$ of the frame $\epsilon_{\alpha}{ }^{\mu}$. The other components $\epsilon_{i}{ }^{\mu}$, $i=1,2,3$, are determined such that they span the tangent plane. Now we can define the dual frame $e^{\alpha}{ }_{\mu}$ in the cotangent bundle using the inverse Gauss map (or inverse Legendre transform in the massive case). We define $e^{0}{ }_{\mu}=G^{-1}\left(\epsilon_{0 \mu}\right)$ and the rest of the frame $e_{\mu}^{i}, i=1,2,3$, is defined as spanning the rest of the cotangent plane, so that $e^{\alpha}{ }_{\mu}$ defines a frame in the cotangent plane. To have a physical notion of energy $E$ and 3-momentum, we need to project the momentum $p_{\mu}$ on $e$. This point is actually often forgotten in the literature. The modified dispersion relation expressed in terms of energy and 3-momentum is then recovered from $\mathcal{M}\left(x, \kappa, E e^{0}+p_{i} e^{i}\right)=0$.

We emphasize that in this approach the notion of energy or 3-momentum is defined in terms of a frame in the cotangent bundle, defined itself in terms of an observer frame and the (inverse) Gauss map. Contrary to the usual approach in QG phenomenology, where one defines arbitrarily the notion of energy, without specifying the notion of frame observer or having a good control on it.

Performing the Gauss map at the level of the action, we obtain its expression in the tangent bundle,

$$
\beta=\int \mathrm{d} \tau \lambda \mathcal{M}^{\sharp}(x, \dot{x}, \kappa) .
$$

$\mathcal{M}^{\sharp}(x, \dot{x})$ is a homogeneous function of $\dot{x}$, of degree different than $\mathcal{M}$, in general. In the standard electromagnetic case on $\mathbb{R}^{4}$, given in terms of (3.5), we recover $\mathcal{M}^{\sharp}(x, \dot{x})=\sqrt{\dot{x}^{\mu} \eta_{\mu \nu} \dot{x}^{\nu}}$. In this special case, the Minkowski metric is recovered with

$$
\eta_{\mu \nu}=\frac{\partial^{2}}{\partial \dot{x}^{\mu} \dot{x}^{\nu}}\left(\mathcal{M}^{\sharp}(x, \dot{x})\right)^{2}=\frac{1}{2} \frac{\partial^{2}}{\partial \dot{x}^{\mu} \dot{x}^{\nu}}\left(\dot{x}^{\mu} \eta_{\mu \nu} \dot{x}^{\nu}\right) \text {. }
$$

If the polynomial $\mathcal{M}^{\sharp}(x, \dot{x})$ is more general, one obtains a metric

$$
g_{\mu \nu}(x, \dot{x})=\frac{\partial^{2}}{\partial \dot{x}^{\mu} \dot{x}^{\nu}}\left(\mathcal{M}^{\sharp}(x, \dot{x})\right)^{2},
$$

which depends both on the position $x$ and the vector $\dot{x}$. This type of metric is called Finsler metric. Riemann was actually aware of this possible extension (i.e. a metric which depends also on vectors) of the metric, but it is only in 1917 that Finsler explored this generalization for his $\mathrm{PhD}$ thesis. This generalized notion of metric can be seen as the rigorous implementation of the notion "rainbow metric" [180].

The notion of a Lorentzian Finsler metric is still a matter of discussion in the Finsler community. The most recent proposals that have been developed concurrently with the approach recalled above can be found in [213,219].

\footnotetext{
${ }^{5}$ Note that we invert the notation of the frames with respect to [219], between $T \mathbb{R}^{4}$ and $T^{*} \mathbb{R}^{4}$.

${ }^{6}$ Hence the difference between massless and massive cases.
} 
The reader could argue that it might not be possible to identify a well defined (invertible) Gauss map or Legendre transform in general. However if few natural assumptions are added, [219] pointed out that the Gauss map and the Legendre map are well defined. Let us summarize these assumptions. (Clearly they can be discussed and one could explore the consequences of removing them.)

- The first assumption is one that we made at the beginning: we assumed that the equations encoding the effective dynamics are linear partial differential equations.

- We take as a very reasonable (or conservative) assumption that if we know the initial conditions, we can predict the propagation of the plane-waves at any later time in the semi-classical spacetime. Not having this assumption would make prediction difficult. On the other hand, one can argue that in the full QG regime, the notion of causality could disappear. We assume here that at the semi-classical level we do not see such effects.

- Finally, we expect that the notion of energy $E$ defined using the observer frame should be positive for any observer.

It is quite striking that these three physical assumptions (linearity, predictability, energy positivity) can be translated into very different types of mathematics, such as algebraic geometry and convex analysis. With these assumptions, the Legendre transform (massive case) and the Gauss map (massless case) are well-defined and invertible. Taken together these assumptions put strong constraints on the possible shape of the covariant dispersion relation $\mathcal{M}(x, \kappa, k)$. [219] showed that some well-known QG motivated modified dispersion relations (such as the Gambini-Pullin's [101]) actually do not satisfy some of the above assumptions. This means that these MDR cannot be understood in this setting and it is quite unlikely that they can be physically interpreted in the context of Lorentz symmetry breaking. For the full details we refer to the [219], the presentation of necessary mathematics would go beyond the scope of this review.

To conclude this quick overview of the Finsler framework, we hope we have conveyed to the reader that there exist more general metric structures than the Lorentzian ones, which seem to be natural candidates to encode the semi-classical QG effects. This mathematical framework has been introduced fairly recently in the QG phenomenology framework [109]. Even more recently, [219] has shown that this framework can be very much constrained from mathematical arguments so that we do not have to explore blindly in every direction what we can do. There is a nice mathematical framework to guide us, which awaits to be further developed. Finally, (some of) the Lorentz symmetries are broken in the Finsler geometry framework. There is no known way to accommodate for spacetime symmetries consistent with the scales present in the modified dispersion relation. This is consistent with the analogy of a medium, which will in general contain some preferred direction and/or scale.

\subsection{Non-linear realization of Poincaré symmetries}

Besides the obvious way to introduce an invariant scale by choosing a preferred reference frame and so sacrificing Lorentz invariance, it is possible to keep the relativity principle intact in presence of a second invariant quantity, a Planck scale $\kappa$ in addition to the speed of light. One does so by modifying both the transformation laws from one inertial system to another, and the law of energy-momentum conservation in such a way that a MDR become compatible with observer independence. This can be achieved through a non-linear realization of (some of) the Poincaré symmetries $[179,181]$. This is a first attempt to describe deformed symmetries since in this framework, the realization of the full Poincaré symmetries in spacetime is not clear.

In the papers $[179,181]$, Magueijo and Smolin do not make any specific assumption regarding the structure of momentum space. It can be flat or curved, it is left open. To fix the notations, 
define first the momentum $\pi$ and the infinitesimal boost which is realized as $J_{0 i}=\pi_{0} \partial_{\pi_{i}}-\pi_{i} \partial_{\pi_{0}}$. It acts linearly on $\pi$

$$
J_{0 i} \triangleright \pi_{j}=\delta_{i j} \pi_{0}, \quad J_{0 i} \triangleright \pi_{0}=-\pi_{i} .
$$

We introduce then a map $U_{\kappa}: \mathbb{R}^{4} \rightarrow \mathbb{R}^{4}$ so that one can define the non-linear realization $K_{0 i}$ of the boosts $J_{0 i}$

$$
K_{0 i}=U_{\kappa} J_{0 i} U_{\kappa}^{-1} .
$$

The choice of $U_{\kappa}$ is such that the non-linear boost $K_{0 i}$ still satisfies the Lorentz algebra

$$
\left[K_{i}, K_{j}\right]=\epsilon_{i j}^{k} J_{k}, \quad\left[J_{i}, K_{j}\right]=\epsilon_{i j}^{k} K_{k}, \quad\left[J_{i}, J_{j}\right]=\epsilon_{i j}^{k} J_{k},
$$

where $J_{i}$ encodes the infinitesimal rotations. An example is given by

$$
U_{\kappa}\left[\pi_{0}\right]=e^{\frac{\pi_{0}}{\kappa} \pi^{\mu} \partial_{\pi}^{\mu}}, \quad \text { so that } \quad U_{\kappa}\left[\pi_{0}\right]\left(\pi_{\mu}\right)=\frac{\pi_{\mu}}{1-\frac{\pi_{0}}{\kappa}} .
$$

Note that this map is not unitary. In this example, we have a maximum energy $\kappa$ as one can check by applying a boost on $\pi_{\mu}$. This specific non-linear realization leaves invariant the modified dispersion relation

$$
\mathcal{M}(\kappa, \pi)=\frac{\pi^{2}}{\left(1-\frac{\pi_{0}}{\kappa}\right)^{2}} .
$$

Clearly other choices of non-linear realizations can be performed. They are simply constrained to satisfy (3.7) and can be chosen to implement a maximum energy or a maximum $3 \mathrm{~d}$ momentum.

Another way to present this proposal is to consider a momentum $p_{\mu}=U_{\kappa}\left(\pi_{\mu}\right)$ as the measured "physical" momentum. The meaning of (3.6) is that first we go to the linear auxiliary variable $\pi$, perform the boost transformation, then deform back to the physical variable $p$. The physical meaning of the variable $\pi$ is then not very clear.

The case of constructing multiparticles has been discussed in this context. Once again, the idea is to use the linear momentum $\pi$ to induce the sum of the physical momenta $p$. A preliminary proposal was

$$
\left(p^{(1)} \oplus p^{(2)}\right) \equiv U_{\kappa}\left(U_{\kappa}^{-1}\left(p^{(1)}\right)+U_{\kappa}^{-1}\left(p^{(2)}\right)\right) .
$$

This proposal however suffered from an immediate drawback: the "soccer ball problem". If it makes sense that a fundamental particle has a momentum bounded by the QG scale, large systems (such as a soccer ball) have doubtlessly a momentum larger than the QG scale. For instance a flying mosquito has a momentum bigger than the QG scale. The sum in (3.8) is such that the total momentum of two particles will still be bounded by the QG scale. The solution to this problem is to have a rescaling of the QG scale so that in the large limit, we can deal with systems which have larger momentum than $\kappa$. Magueijo and Smolin therefore proposed to deal with a sum

$$
\left(p^{(1)} \oplus p^{(2)}\right) \equiv U_{2 \kappa}\left(U_{\kappa}^{-1}\left(p^{(1)}\right)+U_{\kappa}^{-1}\left(p^{(2)}\right)\right) .
$$

This is a rescaling of the Planck scale implemented by hand. One should find a justification for such rescaling by considering a more complete model. Different arguments have been proposed in $[110,181]$. We note, en passant, that this addition of momenta is commutative, since it is based on the standard commutative addition $\pi_{1}+\pi_{2}$. This approach is therefore not equivalent to the non-commutative approach, which we will discuss later. 
To reconstruct spacetime, Magueijo and Smolin propose to see the coordinates as the infinitesimal translations on momentum space. In the specific example they chose, the coordinates are commutative for the Poisson bracket

$$
x_{0}=t=1-\left(1-\frac{\pi_{0}}{\kappa}\right)\left(\left(1-\frac{\pi_{0}}{\kappa}\right) \partial_{\pi_{0}}-\frac{\pi_{i}}{\kappa} \partial_{\pi_{i}}\right), \quad x_{i}=\left(1-\frac{\pi_{0}}{\kappa}\right) \partial_{\pi_{i}} .
$$

They are however functions over the full phase space and not only in configuration space. Their physical meaning is not very clear. The definition of the translations in spacetime is not given explicitly. For a recent attempt to formulate a particle-dependent, "non-universal DSR", see [17].

\subsection{Modified reference frame}

This approach tries to provide a physical meaning to the different momenta $p, \pi$ introduced in the previous subsection. In $[14,15,165]$, the authors recall that to measure the momentum of a particle $\pi_{\mu}$, we actually use a reference frame $e_{a}{ }^{\mu}, a=0, \ldots, 3$. It is the local inertial frame which can be constructed at every point of the spacetime manifold from the metric $g_{\mu \nu}$ through $g_{\mu \nu}=e_{a}{ }^{\mu} e_{b}{ }^{\nu} \eta^{a b}$. The outcome of the measurement is then $p_{a}=e_{a}{ }^{\mu} \pi_{\mu}$. In standard Minkowski spacetime, we have $e_{a}{ }^{\mu} \sim \delta_{a}{ }^{\mu}$ so that $\pi_{a} \sim p_{a}$ Two reference frames $e_{a}{ }^{\mu}, \bar{e}_{a}{ }^{\mu}$ are related by a Lorentz transformation $\Lambda_{b}{ }^{a}$ if $\bar{e}_{b}{ }^{\mu}=\Lambda_{b}{ }^{a} e_{a}^{\mu}$. This transformation induces the standard linear realization of the Lorentz transformation on $p_{a}$

$$
p_{a}=e_{a}{ }^{\mu} \pi_{\mu} \rightarrow \bar{p}_{b}=\bar{e}_{b}{ }^{\mu} \pi_{\mu}=\Lambda_{b}{ }^{a} e_{a}^{\mu} \pi_{\mu}=\Lambda_{b}{ }^{a} p_{a} .
$$

The first idea - proposed in [165] - is to argue that due to some QG effects, the measured momentum $p$ will be a non-linear function of the components $e_{a}{ }^{\mu} \pi_{\mu}$

$$
p_{b}=f_{b}\left(e_{a}^{\mu} \pi_{\mu}, \kappa\right) .
$$

Changing frame by performing a Lorentz transformation on $e$ as in (3.9) will clearly lead to a non-linear realization $\tilde{\Lambda}$ of the Lorentz transformation on $p$.

When constructing explicitly some models to implement this idea, the actual relationship between momentum and frame has been modified in [14,15]. Instead of (3.10), the measured momentum should be defined with respect to an effective tetrad $E^{\mu}{ }_{\alpha}(e, \pi, \kappa)$. In this sense, this is a picture close to the Finsler geometry approach (cf. Section 3.4) or Smolin's derivation from LQG (cf. Section 3.3.3), since the frame is now momentum dependent. An important assumption is that the map relating a trivial frame $e$ with the effective frame $E$

$$
e^{\mu}{ }_{\alpha} \stackrel{\mathcal{U}_{\kappa}}{\longrightarrow} E^{\mu}{ }_{\alpha}(e, \pi, \kappa)
$$

should be reversible, at least in some approximation. When the fluctuations have appropriate symmetries, the map $\mathcal{U}_{\kappa}$ takes the simple form $E^{\mu}{ }_{\alpha}=F(e, \pi, \kappa) e^{\mu}{ }_{\alpha}$, and $F(e, \pi, \kappa) \rightarrow 1$ when $\kappa \rightarrow \infty$. For example, the Magueijo-Smolin dispersion relation $[179,181]$

$$
\frac{p_{0}^{2}-p^{2}}{1-\frac{p_{0}}{\kappa}}=m^{2}
$$

can be expressed as $p_{\alpha}=F(e, \pi, \kappa) e^{\mu}{ }_{\alpha} \pi_{\mu}$ with

$$
F(e, \pi, \kappa)=\frac{1}{1-\frac{\pi_{\mu} e^{\mu} 0}{\kappa}} .
$$


Lorentz transformations act linearly on the tetrad field $e_{\alpha}$, so their action $\tilde{\Lambda}$ on the effective tetrad $E_{\alpha}$ is specified by the following commutative diagram

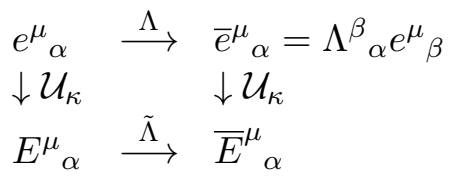

This induces a non-linear transformation of the measured momentum $p$,

$$
p_{\alpha}=\pi_{\mu} E^{\mu}{ }_{\alpha} \rightarrow p_{\alpha}^{\prime}=\pi_{\mu} \bar{E}^{\mu}{ }_{\alpha}=\pi_{\mu} \mathcal{U}_{\kappa}\left(\Lambda \cdot \mathcal{U}_{\kappa}^{-1}(E)\right) .
$$

Different proposals argue that QG fluctuations lead to an effective frame $E^{\mu}{ }_{\alpha}(e, \pi, \kappa)[15,108]$. We have recalled above in Section 3.3.3 Smolin's argument in this sense. Another motivation comes from models from quantum information theory [113]. From the QG perspective it is natural to consider that frames should be quantized. Some physical aspects of the use of quantum reference frame can be explored using finite-dimensional systems, such as spin systems. For example, we can use three quantum spins $\vec{J}_{a}, a=1,2,3$, as $3 \mathrm{~d}$ reference frame and look at the projection of another quantum spin $\vec{S}$ in this frame. The corresponding observable is then

$$
\mathfrak{S}_{a}=\vec{S} \cdot \vec{J}_{a}=S_{i} J^{i}{ }_{a}
$$

We can take by analogy with the QG semi-classical limit a semi-classical frame, that is we consider the reference frame $\vec{J}_{a}$ given in terms of coherent states $|\psi\rangle$. We have therefore the semi-classical reference $\left\langle\psi\left|\vec{J}_{a}\right| \psi\right\rangle \equiv\left\langle\vec{J}_{a}\right\rangle$. We consider the spin $\vec{S}$ projected in this semi-classical frame

$$
\tilde{\mathfrak{S}}_{a}=\vec{S} \cdot\left\langle\overrightarrow{J_{a}}\right\rangle
$$

A priori the frame $\left\langle\vec{J}_{a}\right\rangle$ is independent of the system $\vec{S}$. However it was shown [217] that consecutively measuring the observable $\mathfrak{S}_{a}$ leads to a kickback of the system on the frame, which state then becomes dependent on the system $\vec{S}$. Hence consecutive measurements induce the map

$$
\left\langle\overrightarrow{J_{a}}\right\rangle \rightarrow\left\langle\overrightarrow{J_{a}}\right\rangle(\vec{S}), \quad a=1,2,3 .
$$

This is the analogue of the deformation (3.11). It was shown that this map can be decomposed as a rotation together with some decoherence [217]. The decoherence part is non invertible, so the map (3.12) is not properly reversible. It is reversible only in the approximation where the decoherence can be neglected. For further details we refer to $[106,113]$.

\subsection{Non-commutative space-time}

Non-commutative spaces can be understood as geometries where coordinates become operators. In this sense we move away from the phase space structure we discussed earlier and use the quantum setting. Non-commutative geometries have been mostly introduced by mathematicians and are therefore very well defined mathematically. In general one uses the algebraic concepts of Hopf algebra and so on. In order to restrict the amount of material, we shall present only a pedestrian overview of the topic and refer the more curious reader to the relevant references.

Historically, one of the first examples of non-commutative space is due to Snyder [250] who tried to incorporate the Planck length in a covariant way, i.e. without breaking the Lorentz symmetries. The idea is simple and follows similar philosophy as in the LQG case. Space coordinates can be "discretized" if they are operators with a discrete spectrum. Snyder used 
the subspace generated by the infinitesimal de Sitter boosts $J_{4 \mu}$, of the Lie algebra $\mathfrak{s o}(4,1)$ generated by the elements $J_{A B}$ to encode spacetime. The coordinates are

$$
X_{\mu}=\frac{1}{\kappa} J_{4 \mu}, \quad \text { such that } \quad\left[X_{\mu}, X_{\nu}\right]=\frac{1}{\kappa^{2}} J_{\mu \nu} \in \mathfrak{s o}(3,1) .
$$

The spatial coordinates $X_{i}=\frac{1}{\kappa} J_{4 i}$ are represented as (infinitesimal) rotations and therefore have a discrete spectrum. The time coordinates has instead a continuum spectrum. From the definition of the Lie algebra $\mathfrak{s o}(4,1)$, there is a natural action of the infinitesimal Lorentz transformations on $X_{\mu}$,

$$
\left[J_{\mu \nu}, X_{\alpha}\right]=\left[J_{\mu \nu}, \frac{1}{\kappa} J_{4 \alpha}\right]=\eta_{\mu \alpha} X_{\nu}-\eta_{\nu \alpha} X_{\mu} .
$$

Hence it is possible to have a discrete structure with Lorentz symmetry implemented. By assumption, momentum space is not flat but curved: it is de Sitter space instead of the standard hyperplane $\mathbb{R}^{4}$. Snyder picked a choice of coordinates on the de Sitter space such that Lorentz symmetries are implemented. Considering the embedding of de Sitter in $\mathbb{R}^{5} \ni \pi^{A}$ given by $\pi^{A} \eta_{A B} \pi^{B}=-\kappa^{2}$, Snyder picked

$$
p^{\mu}=\frac{\pi^{\mu}}{\pi^{4}}, \quad \pi^{4}=\frac{1}{\sqrt{1-\frac{p^{2}}{\kappa^{2}}}} .
$$

The dispersion relation would be the classic one, since the Lorentz symmetries are the usual ones,

$$
p^{2}=m^{2} .
$$

Note that his choice of coordinates is actually very close to the one picked up in special relativity. Indeed in this context, we have the space of $3 \mathrm{~d}$ speeds which becomes the hyperboloid

$$
V^{\mu} \eta_{\mu \nu} V^{\nu}=c^{2}
$$

where the natural choice of coordinates is

$$
v^{i}=\frac{V^{i}}{V^{0}}, \quad V^{0}=\frac{1}{\sqrt{1-\frac{v^{2}}{c^{2}}}} .
$$

Snyder did not discuss the addition of momenta. However since momentum space is now curved it is clear that the addition has to be non-trivial. We can take inspiration from the addition of velocities in special relativity: it is given in terms of a product which is non-commutative and non-associative. The space of velocities is then not a group but instead a K-loop or a gyrogroup [259].

A similar addition can be defined for the Snyder case [107]. The addition of momenta will then be non-commutative and non-associative. This means that the notion of translations becomes extremely non-trivial. We shall come back on this structure when recalling the recent results of "relative locality" [23]. For different approaches to deal with Snyder's spacetime we refer to [111] and the references therein.

Snyder's spacetime is related to the Doplicher-Fredenhagen-Roberts (DFR) spacetime, which was constructed independently of Snyder's spacetime, using different tools [88],

$$
\left[X_{\mu}, X_{\nu}\right]=\frac{1}{\kappa} Q_{\mu \nu}, \quad\left[Q_{\mu \nu}, X_{\alpha}\right]=0=\left[Q_{\mu \nu}, Q_{\alpha \beta}\right] .
$$

The operators $Q_{\mu \nu}$ can be seen as (commutative) coordinates on some extra dimensional space. 
This space can be seen as an abelianized version of Snyder spacetime [75,111]. Starting from Snyder's commutation relations (i.e. the algebra $\mathfrak{s o}(4,1)$ ), consider $Q_{\mu \nu}=\frac{1}{\kappa^{\prime}} J_{\mu \nu}$ and the limit $\kappa, \kappa^{\prime} \rightarrow \infty$ with $\frac{\kappa^{\prime}}{\kappa^{2}}=\frac{1}{\tilde{\kappa}}$ fixed. It is not complicated to see that Snyder's algebra gives an algebra isomorphic to the DFR algebra in this limit.

Both Snyder's and DFR's non-commutative spaces can also be understood as non-commutative spaces embedded in a bigger non-commutative space of the Lie algebra type [111]. Once again in this case there is no deformation of the Lorentz symmetries, the modified dispersion relation is again the standard one. However there are non trivial uncertainty relations which implement a notion of minimum "area" [88],

$$
\triangle x_{0}\left(\triangle x_{1}+\triangle x_{2}+\triangle x_{3}\right) \geq \kappa^{-2}, \quad \triangle x_{1} \triangle x_{2}+\triangle x_{2} \triangle x_{3}+\triangle x_{3} \triangle x_{1} \geq \kappa^{-2} .
$$

The famous Moyal spacetime

$$
\left[X_{\mu}, X_{\nu}\right]=\frac{i}{\kappa^{2}} \theta_{\mu \nu} \mathbb{1}, \quad\left[X_{\mu}, \mathbb{1}\right]=0
$$

is as an example of the DFR spacetime, as amy abe seen by projecting $Q_{\mu \nu}$ onto a specific eigenspace [91]. The Moyal spacetime is the most studied of the non-commutative spacetimes. There exists a huge literature on the physics in this spacetime, we refer only to [44,129], where the relevant literature can be found.

The tensor $\theta_{\mu \nu}$ is a tensor made of c-numbers. It is invariant under translations and Lorentz transformations (unlike the $Q_{\mu \nu}$ in the DFR space which transform under Lorentz transformations). It is not complicated to check that (3.13) transforms covariantly under an infinitesimal translation. However the case of the (infinitesimal) Lorentz transformations is more tricky since

$$
J_{\mu \nu} \triangleright\left[X_{\alpha}, X_{\beta}\right]=i\left(\eta_{\nu \alpha} \theta_{\mu \beta}-\eta_{\mu \alpha} \theta_{\nu \beta}+\eta_{\nu \beta} \theta_{\alpha \mu}-\eta_{\mu \beta} \theta_{\alpha \nu}\right) \mathbb{1} \neq \frac{1}{\kappa^{2}} J_{\mu \nu} \triangleright\left(i \theta_{\alpha \beta} \mathbb{1}\right)=0,
$$

where we have used $J_{\mu \nu} \triangleright X_{\alpha}=\eta_{\nu \alpha} X_{\mu}-\eta_{\mu \alpha} X_{\nu}$ and the Leibniz law

$$
J_{\mu \nu} \triangleright\left(X_{\alpha} X_{\beta}\right)=\left(J_{\mu \nu} \triangleright X_{\alpha}\right) X_{\beta}+X_{\alpha}\left(J_{\mu \nu} \triangleright X_{\beta}\right) .
$$

One can then say that the Lorentz symmetries are broken since the non-commutativity is not consistent with the change of frame. Most often in the study of Moyal spacetime, this is the chosen perspective. One can also look for a deformation of the action of these Lorentz symmetries, to make them compatible with the non-commutative structure [210]. This deformation can be understood as a modification of the Leibniz law

$$
\begin{aligned}
J_{\mu \nu} \triangleright\left(X_{\alpha} X_{\beta}\right)= & \left(J_{\mu \nu} \triangleright X_{\alpha}\right) X_{\beta}+X_{\alpha}\left(J_{\mu \nu} \triangleright X_{\beta}\right) \\
& -\frac{1}{2} \theta^{\rho \sigma}\left(\left(\left(\eta_{\rho \mu} \partial_{\nu}-\eta_{\rho \nu} \partial_{\mu}\right) \triangleright X_{\alpha}\right) \partial_{\sigma} \triangleright X_{\beta}\right. \\
& \left.+\partial_{\rho} \triangleright X_{\alpha}\left(\left(\eta_{\sigma \mu} \partial_{\nu}-\eta_{\sigma \nu} \partial_{\mu}\right) \triangleright X_{\beta}\right)\right) .
\end{aligned}
$$

It can be extended easily to arbitrary product of functions. With this new Leibniz law, the non-commutativity (3.13) will be consistent with the Lorentz symmetries as one can check.

The proper way to encode this modification is to use algebraic structures such as quantum groups [182]. Indeed, the modified Leibniz law comes from a non-trivial coproduct structure. There exists therefore a deformation of the Poincaré group that is the symmetry group of Moyal's spacetime and as such this spacetime can be seen as a flat non-commutative spacetime.

There is no deformation of the action of the Lorentz transformations on the momenta so that it is the usual mass-shell relation that one considers. Furthermore since the translations are not modified either, there is no modification of the addition of momenta. We refer to [44,129] and references therein for some discussions of the phenomenology of this space. 
An important example of non-commutative space for QG phenomenology is the $\kappa$-Minkowski spacetime. We also mention his cousin the $\kappa^{\prime}$-Minkowski spacetime. Their non-commutative structures are defined, respectively, as

$$
\begin{array}{ll}
{\left[X_{0}, X_{i}\right]=\frac{1}{\kappa} X_{i},} & {\left[X_{i}, X_{j}\right]=0, \quad i, j=1,2,3,} \\
{\left[X_{1}, X_{\mu}\right]=\frac{1}{\kappa} X_{\mu}, \quad\left[X_{\mu}, X_{\nu}\right]=0, \quad \mu, \nu=0,2,3 .}
\end{array}
$$

In the $\kappa$-Minkowski case, it is the time coordinate that is not commutative with the space coordinates, whereas in the $\kappa^{\prime}$-Minkowski case, it is a space coordinate (here $X_{1}$, but clearly other cases can be considered) that is non-commutative with the others. They encode the most rigorous models of deformed special relativity ${ }^{7}$ (DSR).

These non-commutative spacetimes provide examples where both the Lorentz transformations and the translations are deformed. Let us focus on the case of translations in $\kappa$-Minkowski. The commutator cannot be covariant under an infinitesimal translation if we use the usual Leibniz law. Indeed, the commutator is invariant if we use the standard Leibniz law

$$
\partial_{\mu} \triangleright\left(X_{\alpha} X_{\beta}\right)=\eta_{\alpha \mu} X_{\beta}+\eta_{\beta \mu} X_{\alpha} \quad \Longrightarrow \quad \partial_{\mu} \triangleright\left[X_{\alpha}, X_{\beta}\right]=0 .
$$

But due to (3.14), and $\partial_{\mu} \triangleright X_{\alpha}=\eta_{\mu \alpha}$, we see we get for example

$$
\partial_{j} \triangleright\left[X_{0}, X_{i}\right]=0=\eta_{i j},
$$

which is a contradiction. We can then argue either that the translation symmetries are broken or that we can modify the Leibniz law in order to have an action of the translations compatible with the non-commutative structure. One can check that the modified Leibniz law on the spatial derivatives (the time derivative satisfies the usual Leibniz law)

$$
\begin{aligned}
& \partial_{i} \triangleright\left(X_{\alpha} X_{\beta}\right)=\left(\partial_{i} \triangleright X_{\alpha}\right) X_{\beta}+\left(e^{\frac{\partial_{0}}{\kappa}} \triangleright X_{\alpha}\right)\left(\partial_{i} \triangleright X_{\beta}\right), \\
& e^{\frac{\partial_{0}}{\kappa}} \triangleright X_{\alpha}= \begin{cases}X_{\alpha}, & \alpha \neq 0, \\
X_{0}+1 / \kappa, & \alpha=0\end{cases}
\end{aligned}
$$

does the job. As in the Moyal case, there is a deformation of the Poincaré group which encodes such a modification. This quantum group is called the $\kappa$-Poincaré group $[170,184]$. When one identifies the translations with momenta, the alter-ego of the modified Leibniz law is a modified addition law of momenta

$$
(p \oplus q)_{0}=p_{0}+q_{0}, \quad(p \oplus q)_{i}=p_{i}+e^{p_{0} / \kappa} q_{i},
$$

which comes from the non-trivial coproduct of the $\kappa$-Poincaré quantum group. Just as in the Snyder case, the momentum space in the $\kappa$-Minkowski case is given by the de Sitter space. However in this case, the de Sitter space $d S \sim \mathrm{SO}(4,1) / \mathrm{SO}(3,1)$ is equipped with a group product, so that the momentum addition (3.15) is non-commutative but associative. The group structure can be obtained by factorizing the group $\mathrm{SO}(4,1)=G \cdot \mathrm{SO}(3,1)$, where $G$ is the group (actually two copies of the group called $\mathrm{AN}_{3}$ ) encoding momentum space. This implies that there is an action of the Lorentz group on momentum space $G$ but also a back action of $G$ on $\mathrm{SO}(3,1)[182,184]$. Putting all together, we get a non-linear realization of the Lorentz symmetries on momentum space $\left(N_{i}, R_{i}\right.$ are respectively the boosts and the rotations)

$$
\left[R_{i}, p_{j}\right]=\epsilon_{i j}^{l} p_{l}, \quad\left[R_{i}, p_{0}\right]=0,
$$

\footnotetext{
${ }^{7}$ Unfortunately the name of DSR has been given to many different approaches.
} 


$$
\left[N_{i}, p_{j}\right]=\delta_{i j}\left(\sinh \frac{p_{0}}{\kappa}-\frac{\mathbf{p}^{2}}{2 \kappa^{2}} e^{p_{0} / \kappa}\right), \quad\left[N_{i}, p_{0}\right]=p_{i} e^{p_{0} / \kappa}
$$

Just like for the translations, the compatibility of the non-commutative structure (3.14) with Lorentz transformations implies a deformation of the Leibniz law for the action of the Lorentz transformations [184]. This is also inherited from the $\kappa$-Poincare group. This non-commutative space was one of the first frameworks used to discuss the non-trivial propagation of gammarays [26]. We refer to $[16,28,128,153]$ for a discussion of the phenomenology of this space.

We have presented a set of non-commutative spaces and discussed the realization of the Poincaré symmetries. It is interesting to ask whether all these spaces can be classified. To our knowledge, there are two types of possible classifications:

- The first one is to look at all the possible deformations of the Poincaré group using quantum group techniques, i.e. algebraic techniques. If one identifies the momentum operator with the translation, this provides a set of different momentum spaces, from which we can determine by duality the dual spacetime. Therefore determining the deformations of the Poincaré group specifies momentum spaces and the relevant non-commutative spaces. In $4 \mathrm{~d}$ (as well as in 3d), this classification was performed [215] and a set of 21 deformations have been identified. The Moyal spacetime and the $\kappa$ - and $\kappa^{\prime}$-Minkowski spaces ${ }^{8}$ are of course among them. However, Snyder's spacetime is not among them since it is related to a non-associative deformation. This classification is hence missing some (at least historically) interesting spaces.

Note that among this classification, there is a number of momentum spaces that appear as Lie groups. It would be interesting to check whether the full set of $4 \mathrm{~d}$ Lie groups appear in this classification.

Finally, we emphasize, that a priori all these 21 deformations are equally valid candidates for a non-commutative description of QG semi-classical flat spacetime.

- The second approach is geometric in nature but it has not been performed in detail yet. It consists in classifying all the possible $4 \mathrm{~d}$ smooth loops (i.e. manifold equipped with a product, a unit and an inverse) that carry an action of the Lorentz group. This would provide a classification of possible momentum spaces, and one would need to check if one can find some action of the Lorentz group on them to get the relevant Poincaré group deformation.

Different works have pointed out that classifying the loops can be seen as a classification of the possible connections on some given manifold (especially in the homogenous case). For example on the de Sitter space as momentum space, one can have a group structure $G=A N_{3}$ which would give rise to $\kappa$-Minkowski spacetime, or a K-loop structure, giving rise to Snyder's spacetime $[23,93,107]$. These different choices amount to different types of connections on the de Sitter space.

We should note however that with this geometric classification, we would get the nonassociative spaces but we would miss momentum spaces of the quantum group or of the Moyal type. Hence the algebraic and geometric approaches are complementary.

The Moyal and $\kappa$-Minkowski spacetimes have attracted most attention in the QG phenomeno$\operatorname{logy} /$ non-commutative communities. Among these two, the Moyal spacetime is the best understood, probably because the non-commutativity is in fact of a simpler nature (a twist) than $\kappa$-Minkowski. Historically Moyal spacetime was also identified before $\kappa$-Minkowski. In the

\footnotetext{
${ }^{8}$ Historically, the $\kappa$-Poincaré deformation was discovered through two different approaches: the Inönü-Wigner contraction of the quantum group $\mathrm{SO}_{q}(4,1)$ [170] and the bicrossproduct construction [184].
} 
LQG community, the $\kappa$-Minkowski case is the most popular example, though $\kappa^{\prime}$-Minkowski also appears in 3d QG [199].

Non-commutative structures appear in the LQG context through the failure of area operators acting on intersecting surfaces to commute [32]. Currently, there is no derivation of non-commutative "coordinates" in 4d LQG. We shall come back in Section 4.3.2 on a derivation of non-commutative field theory for matter using the group field theory approach.

\subsection{Relative locality}

This recent development in the construction of effective theories proposes a tentative interpretation of non-commutative coordinates at the classical level. The basic claim of "Relative Locality" (RL) is that we live in phase space, not in space-time. In this view, rather than a global space-time, there are only energy-momentum dependent cotangent spaces (interpreted as spacetime) of the curved momentum space $\mathcal{M}$, a concept first proposed in [180]. RL attempts to describe "classical, non-gravitational quantum gravity effects", i.e. remnants of QG, when gravity and quantum theory are switched off by going to the limits $\hbar \rightarrow 0$ and $G \rightarrow 0$. In this limit the Planck length goes to zero as well, whereas the Planck mass $\kappa$ is finite. In this way an invariant energy scale is obtained, but not an invariant length. The scale $\kappa$ can be introduced by considering a homogeneous momentum space with (constant) curvature $\kappa$. In fact even more general curved momentum spaces can be introduced. If momentum space is not homogeneous of curvature $\kappa$, but of a more general type, the scale $\kappa$ will still appear in the modified dispersion relation or the non-trivial addition in order to have dimensional meaningful quantities. Quite strikingly the structures associated to momentum space - dispersion relation, addition of momenta - can be related to geometric structures on momentum space. Indeed, it can be shown that the metric on momentum space encodes the dispersion relation, whereas the sum of momenta is encoded through a connection (which does not have to be either metric compatible or torsion free or flat).

To be more explicit, consider an event. Following Einstein an event can be seen as the intersection of different worldlines. From a quantum field theory perspective, an event can be seen as a vertex in a Feynman diagram, with a given number of legs. Each leg can be seen as a particle labelled by $J$ and momentum $p_{\mu}^{J}$. In case of a general addition $\oplus$, we can write the total momentum at this vertex as

$$
P_{\mu}^{\mathrm{tot}}=\left(p^{1} \oplus \cdots \oplus p^{N}\right)_{\mu} \approx \sum_{I} p_{\mu}^{I}+\frac{1}{\kappa} \sum_{I<J} \Gamma_{\mu}^{\alpha \beta} p_{\alpha}^{I} p_{\beta}^{J}+\cdots,
$$

with the connection ${ }^{9} \Gamma_{a}^{b c}$ on momentum space.

A non-zero torsion is equivalent to the non-commutativity of the addition, whereas nonassociativity of the addition is equivalent to a connection with non-zero curvature [23,93]. Hence, a Lie algebra type non-commutative space which has therefore a momentum addition (in general) non-commutative but associative will correspond - in this RL context - to a choice of a flat connection with torsion. In particular, the $\kappa$-Minkowski spacetime illustrates this case [125].

The particle $J$ has momentum $p^{J} \in \mathcal{M}$ and position $x_{J} \in T_{p^{J}}^{*} \mathcal{M}$. At the intersection of the worldlines when particles interact, we expect conservation of momenta, that is $P_{\mu}^{\text {tot }}=0$. This means that this event (the vertex) sits in the cotangent space $T_{p=0}^{*} \mathcal{M}$ at the origin of momentum space and has coordinates $z^{\mu}$. To get the vertex interaction in $T_{0}^{*} \mathcal{M}$, we therefore need to parallel transport the (covector) coordinates $x_{J}$ to $T_{0} \mathcal{M}$

$$
\left(\tau_{J}^{-1}\right)^{\mu}{ }_{\nu} x_{J}^{\nu}=z^{\mu}
$$

\footnotetext{
${ }^{9}$ Note in this approach the notation of covectors and vectors are inverse to the notation we are using in general in this review, since the basic manifold is momentum space. Hence a covector will have index up, whereas a vector will have the index down.
} 
where $\tau_{J \nu}^{\mu}$ encodes the parallel transport associated to the particle $J$. In [22], the authors showed that an action for $N$ particles with the constraint implementing momentum conservation $P_{\mu}^{\text {tot }}$ lead to such a construction. There is a unique coordinate $z^{\mu}$ for the interaction vertex and in particular (cf. (3.16))

$$
\tau_{J \nu}^{\mu}=\frac{\partial P_{\nu}^{\mathrm{tot}}}{\partial p_{\mu}^{J}}
$$

The interaction coordinates do not Poisson commute,

$$
\left\{z^{\mu}, z^{\nu}\right\}=\frac{1}{\kappa} T_{\sigma}^{\mu \nu} z^{\sigma}+\frac{1}{\kappa^{2}} R_{\sigma}{ }^{\mu \nu \rho} p_{\rho} z^{\sigma}+\cdots,
$$

$T_{\sigma}{ }^{\mu \nu}$ and $R_{\sigma}{ }^{\mu \nu \rho}$ are respectively the torsion and the Riemann tensor for the connection $\Gamma$ associated to the momenta addition (3.16). These coordinates are therefore interesting candidates for the meaning of the quantum operators encoded in the non-commutative geometry as discussed in Section 3.7. Indeed, if we take the case of a connection with constant torsion but zero curvature, (3.17) becomes the classical analogue of a Lie algebra type non-commutative space. In particular one can retrieve the $\kappa$-Minkowski classical case [125].

The transition to the reference frame of a distant observer is carried out simply by a translation with the infinitesimal form

$$
x_{J}^{\mu} \rightarrow x_{J}^{\mu}+\delta_{\epsilon} x_{J}^{\mu}
$$

where

$$
\delta_{\epsilon} x_{J}^{\mu}=\epsilon^{\nu}\left\{x_{J}^{\mu}, P_{\nu}^{\mathrm{tot}}\right\} \approx b^{\mu}+\frac{1}{\kappa} \epsilon^{\nu} \sum_{I>J} \Gamma_{\nu}^{\mu \beta} p_{\beta}^{I} .
$$

In consequence, for different particles $\delta x_{I}^{\mu}$ is different, so that the translated endpoints of the worldlines do not meet at the vertex and the interaction appears non-local for distant observers. In this way locality becomes relative to a certain extent, as pointed out in $[27,132,135,138,243$, $246]$.

In [23] the emission of a low-energy and a high-energy photon and their absorption by a distant detector as a model for radiation from gamma-ray-bursts is discussed in detail. In RL, the speed of light is an invariant, but the trajectories of photons at different energies with their origin and end points lie in different copies of space-time. To compare them and to calculate a possible time delay between the photons, one must parallel transport the corresponding cotangent spaces into one. Note that, for example for an absorption event, the endpoint of the detector's worldline before the absorption, the origin of its worldline after the absorption and the endpoint of the photon's worldline do not coincide in general. These non-localities at the absorption or emission events are relative, depending on the observer's reference frame, but the resulting time delay in first order,

$$
\Delta T=-\frac{1}{2} T E N^{+++},
$$

is an observer-independent invariant. $T$ is the running time of the high-energy photon in the detector's frame (at rest with the source in the model), $E$ is the photon's energy in this frame and $N^{+++}$denotes the component of the non-metricity tensor $\left(N_{a b c}=\nabla_{a} g_{b c}\right)$ of the connection along the photon direction. In the RL framework current observations $[2,3]$ can be interpreted as implying a bound on non-metricity

$$
\left|N^{+++}\right| \leq \frac{1}{0.6 \kappa} \text {. }
$$


A second effect, derived from the same model is dual gravitational lensing: Two photons with proportional momenta need not propagate into the same directions. When the connection of the curved momentum space has torsion, a rotation angle of

$$
\Delta \theta=\frac{E_{1}+E_{2}}{2}\left|T^{a}\right|
$$

is predicted, where the vector $T^{a}$ arises by projection of the torsion tensor into the direction of the photons' momenta and $E_{i}$ are the photons'energies. Effects of curvature show up in the approximation quadratic in $E / \kappa$ [93]. The framework of $\kappa$-Ponicaré algebras is an example with non-metric connection, zero curvature and non-zero torsion. Further experiments that may measure or bound the geometry of momentum space at order $\kappa^{-1}$ include tests of the linearity of momentum conservation using ultracold atoms [31] and the development of air showers produced by cosmic rays [29].

\subsection{Generalized uncertainty principle}

This approach is connected with the strong gravity regime rather than with the amplification of "low" energy effects. Nevertheless, it has formal similarity with DSR theories. But the concept of the generalized uncertainty principle (GUP) has a physically compelling basis: QG effects should occur at densities comparable with the Planck density, not in the presence of large, extended masses. For a modeling of gravity-caused modifications of scattering processes at extreme energy, see [133]. GUP is tied to the center-of-momentum (c.o.m.) energy of two or more particles, concentrated in a small region in an interaction process, or the energy of one particle in relation to some matter background, like the CMB. When in a scattering process the c.o.m. energy is high enough, so that in the scattering region the energy density comes close to the Planck density, there is significant space-time curvature and gravity is non-negligible. The gravitational influence is described by a local energy dependence of the metric. At this point GUP introduces a split between the momentum and the wave vector of a particle. In the case of two scalar particles scattering in the c.o.m. system the asymptotic momenta are $p^{\mu}$ and $-p^{\mu}$, related linearly to the wave vectors $k^{\mu}=p^{\mu} / \hbar$ in the asymptotic region. The curvature caused by the energy density is described by a dependence of the metric on the wave vector, $g_{\mu \nu}=g_{\mu \nu}(k)$, leading to a modified dispersion relation

$$
k^{\mu} g_{\mu \nu}(k) k^{\nu}=m^{2},
$$

where $m$ is a mass parameter. Relation (3.18) containing higher-order terms in $k, k^{\mu}$ is not a Lorentz vector and will not transform according to standard flat-space Lorentz transformations. Provided we do not assume graviton production, the asymptotic momenta are conserved, but in the interaction region the relation between $p^{\mu}$ and $k^{\mu}$ becomes nonlinear.

Formally, the asymptotic momenta, which are acted upon linearly by the Lorentz group, play a role analogous to that of the pseudo-variables in DSR. But, whereas in DSR the latter ones are mere auxiliary quantities, here they have a clear, distinct physical meaning. The nonlinear variables $k^{\mu}$, on the other hand, play a more or less auxiliary role, in contrast to the nonlinear "physical variables" in DSR.

So far, this is nothing more than an effective description of gravity, when it plays a role in high energy particle interaction. The place where it is encoded in the effective theory is the form of the function $k(p)$, or its inverse, respectively. This function could, in principle, encode Newtonian gravity, GR, or QG. The input from QG that is made here is the existence of a minimal length, $1 / \kappa$. General conditions on the functional dependence of $k$ on $p$ are given in [133]:

1. For energies much smaller than $\kappa$ the usual linear relation is found. 
2. For large energies, $k$ goes asymptotically to $\kappa$, not to infinity.

3. The function is invertible, i.e. it is monotonically increasing.

With these conditions satisfied, $k$ remains bounded when $p$ grows arbitrarily and the (effective) wavelength $\lambda=2 \pi /|k|$ does not decrease below the invariant length. Theories of this type have been examined in various contexts as to their analytical structure and phenomenological consequences $[73,146,175,176]$.

Recalling the quantum mechanical relation $p=\hbar k$, an energy-dependent relation between momentum and wave vector can be formulated as an energy dependence of Planck's constant, $p^{a}=\hbar(p) k^{a}$, thus introducing an additional quantum uncertainty. Such a modification of quantum mechanics was suggested for the first time by Heisenberg [127]. The physical idea is that a sufficiently high energy particle, released in the interaction at a detecting process, curves and disturbs space-time so that an additional position uncertainty arises, which enhances the quantum mechanical one. In this way the accuracy of position measurement is bounded from below by an invariant length scale.

To formulate GUP in terms of commutation relations, we postulate the canonical relation for the wave vector with a coordinate $x$ and derive the relations between coordinates and momenta,

$$
\left[x^{a}, k_{b}\right]=i \hbar \delta_{b}^{a}, \quad\left[x^{a}, p_{b}\right]=i \hbar \frac{\partial p_{b}}{\partial k_{a}} .
$$

This results in the generalized uncertainty relations

$$
\Delta x^{a} \Delta p_{b} \geq \frac{\hbar}{2}\left|\left\langle\frac{\partial p_{b}}{\partial k_{a}}\right\rangle\right|
$$

Comparison with DSR-type theories shows modified dispersion relations as a common feature, in this sense they are almost two sides of the same coin [131]. The interpretation of MDRs, however, is quite controversial. DSR deals with particle propagation in flat space, GUP deals with QG effects in regions with strong gravity. Both approaches could be compared with each other and with the angle operator [190] by calculating corrections to scattering cross sections. A comparison of DSR versus GUP was made in [130], resulting in an opposite influence of DSR and GUP on scattering cross sections.

\subsection{QG decoherence}

Another very general approach, without close relation to a specific QG theory, is decoherence [260]. This framework considers quantum theoretical fluctuations of the Minkowski metric, so the proper time of particles fluctuates at a time scale $\lambda T_{\mathrm{P}}$, a few orders above the Planck time. In matter wave interferometry, described in [260], atoms act as clocks with a very high frequency. When in such an experiment a matter wave is split into two components and recombined, spacetime fluctuations are expected to cause decoherence, a process, where ingoing pure states become mixed by the dissipative action of the fluctuating background. [260] presents one realization and potential observable consequences, which predict a factor $\lambda$ of the order $10^{3}$. Other realizations can be found, for example, in references in [260] and in [145], where impacts on neutrino physics are considered. For an early contribution see [211].

\section{Quantum field theory frameworks}

In this section we review Lorentz symmetry violating EFTs, associated constraints, a possible "combinatoric lever arm" in scattering experiments, and non-commutative field theory. 


\subsection{Constraints on Lorentz violation with effective field theory}

A successful area of quantum gravity phenomenology in recent years is in the area of Lorentz symmetry breaking. There is a well-developed framework, many new effects, and very strong constraints on these effects, even below the Planck scale. However we do not know whether violations of local Lorentz invariance occur in LQG - in fact there are strong arguments suggesting LLI should be preserved as discussed in Section 2.6. The now-extensive body of work provides a firm foundation for future derivations, constraints and observational searches.

When local Lorentz symmetry is broken a cascade of physical effects appear. Whether the LV is via symmetry breaking or by an additional background field, the effects are studied by adding the possible terms with the new Lorentz violating vector and tensor fields. To organize these effects and the associated constraints, the terms in the particle Lagrangian are characterized by their mass dimension.

Without ties to a specific fundamental theory, the minimal Standard Model Extension provides a framework of organizing renormalizable higher dimension terms in the standard model [79]. There is an extensive literature on these effects and the associated constraints. For more on these constraints see [53,150]. See [152] for data tables on the standard model extension parameters, updated annually.

There is a wealth of effects that arise from LV. Some of these include

- sidereal variation in signals as the Earth moves relative to the preferred frame or directions.

- new processes such as photon decay, photon splitting, and vacuum Cerenkov radiation

- shifting of thresholds of allowed processes in LLI physics such as the GZK threshold

- kinematic effects arising from modifications to dispersion relations that accumulate over cosmological distances or over a large number of particles

Work on dimension 5 and 6 operators is more recent and this section will focus on these. For more on work in LV prior to the developments in LQG see the reviews [139,141,163,194]. The primary reference to consult for dimension 5 LV QED is [141].

Effective Field Theory (EFT) is the framework for describing much of the standard model of particle physics. According to the Wilsonian view of renormalization, at the relatively low energies (as compared to the Planck scale in the case of quantum gravity) explored by accelerators, the dominant interactions in the Lagrangian/action are necessarily the relevant terms at these energies. This means that when introducing new effects it is natural to use the EFT framework to model the physics. In this section the EFT framework is used to explore the possible LV dimension 5 operators in QED, yielding cubic modifications to dispersion relations.

Including a time-like background four vector field $u^{\mu}$ in QED Myers and Pospelov found three LV dimension 5 operators that were (i) quadratic in the same field, (ii) gauge invariant, and (iii) irreducible under use of the equations of motion or a total derivative [208]. Due to the background vector field $u^{\mu}$ there is a preferred frame, usually assumed to be the frame in which the cosmic microwave background is isotropic. The resulting operators (up to factors of 2 used here to simplify the dispersion relations) are

$$
\mathcal{L}_{L V}=\frac{\xi}{2 \kappa} u^{\mu} F_{\mu \nu} u \cdot \partial\left(u_{\sigma} \tilde{F}^{\sigma \nu}\right)+\frac{1}{\kappa} u^{\mu} \bar{\psi} \gamma_{\mu}\left(\zeta_{1}+\zeta_{2} \gamma_{5}\right)(u \cdot \partial)^{2} \psi
$$

Recall that since $\kappa=M_{\mathrm{P}}$, the parameters $\xi, \zeta_{i}$ are all dimensionless. These terms violate CPT symmetry. The resulting modified dispersion relations include cubic modifications compared to the LLI case. For photons,

$$
\omega_{ \pm}^{2}=p_{\gamma}^{2} \pm \frac{\xi}{\kappa} p_{\gamma}^{3}
$$


where the signs indicate left and right polarization and the modification depends on the magnitude of the momentum. While, for fermions,

$$
E_{ \pm}^{2}=p^{2}+m^{2}+\eta_{ \pm} \frac{p^{3}}{\kappa}
$$

in which $\eta_{ \pm}=2\left(\zeta_{1} \pm \zeta_{2}\right)$ and the signs are the positive and negative helicity states. The analysis of the free particle states is in [141] where it is shown that the positron helicity states have a relative sign compared to the electron; there are only two fermion parameters $\eta_{ \pm}$.

When the sign of the modification is negative (positive) the energy decreases (increases) relative to the LLI theory. Thus the curve $E(p)$ flattens (steepens) out at high energies. This strongly affects the process rate, thresholds, and the partitioning of momentum.

Comparing these dispersion relations to those found with the heuristic LQG computations in Section 3.3.1, (3.2) and (3.3) we see that the dimension 5 CPT-odd terms arose in that calculation, giving similar modifications in the dispersion relations when

$$
\eta_{ \pm}=\mp \frac{\kappa_{7}}{2}\left(\frac{1}{L \kappa}\right)^{1+\Upsilon} \quad \text { and } \quad \xi \simeq 4 \theta_{8} .
$$

(Recall that $L$ is the characteristic scale of the semi-classical state, above which the geometry is flat.) The photon MDR is essentially identical. The fermion MDR has only one helicity parameter and has an additional suppression due to the scaling $L \kappa$. Further work on LQG coherent states would clarify this scaling and illuminate the additional scaling arising in the preliminary calculation, perhaps even show an associated custodial symmetry. The dimension 6 terms are identical to the EFT framework. However, none of these operators considered have been derived unambiguously from the framework of LQG.

The EFT analysis has been extended in a variety of ways. Hadrons [174] and even heavy nuclei [237] were included, the framework has been folded into the Standard Model Extension [151]. Additionally, the analysis was generalized to arbitrary 4-vectors is [124]. In the original work of Myers and Pospelov the 4-vector $u^{\mu}$ only had a non-vanishing time component in the preferred frame, chosen due to the stringent constraints on spatial anisotropy set by clock comparison and spin-polarized matter experiments. Given the remarkable bounds on the parameters in the Myers-Pospelov model discussed below, the authors of [124] work with a model of anisotropic media and show that the bounds in the Myers-Pospelov model may be weakened when analyzed in the more general, anisotropic model.

In the context of LV EFT a variety of new phenomena occur $[140,141]$

- New processes, forbidden in the usual LLI theory, can occur.

- Thresholds for processes in the LLI theory can shift.

- Upper thresholds can occur; momenta can be high enough so that processes turn off.

The modifications become important when the mass term is comparable to the modification. Thus for dimension 5 operators the cubic corrections become important when $p_{\text {crit }} \approx\left(m^{2} M_{\mathrm{P}}\right)^{1 / 3}$. Since the effects arise at high momentum many calculations are done for $m \ll p \ll \kappa$, which allows for considerable simplification. We'll use $\simeq$ to denote results in this "high momentum" limit.

It is worth keeping in mind that this framework excludes a wider class of theories, for instance those that contain violations of local energy-momentum conservation. This wider arena was briefly reviewed in Section 3.10. Nevertheless at some energy scale the new theories must match known results and fit within the EFT framework so at lower energies the framework is an excellent approximation. The EFT framework also has the advantage that clear physical predictions can be made such as in the cases of particle process thresholds, where the rates of new particle processes determine the thresholds [141]. 


\subsubsection{Physical effects giving current constraints}

To give a flavor of the nature of the constraints in the next subsections we sketch the derivations of the current tightest constraints on dimension $5 \mathrm{LV}$ QED. There are many other effects but in the interest of reviewing those that give both a sense of the calculations and the strongest constraints, we focus on vacuum birefringence and photon decay. The field is comprehensively reviewed in [141], to which the reader should turn for details. A recent update is [163].

The first phenomenon, arising from birefringence, is purely kinematical. The remaining constraints on dimension $5 \mathrm{LV}$ QED are dynamical in the sense that the dynamics of EFT is employed to derive the constraints. The effects usually involve an analysis of process thresholds.

Threshold constraints require answers to two questions, "Is the process allowed?" and "Does it occur?" A typical process involves a decay of an unstable particle into two particles. In the EFT framework we have usual field theory tools at our disposal so we can compute the rate of decay using the familiar expression from field theory. Denoting the outgoing momenta $p^{\prime}$ and $p^{\prime \prime}$ and helicity with $s$ and the matrix element by $M\left(p, s, p^{\prime}, s^{\prime}, p^{\prime \prime}, s^{\prime \prime}\right)$ the rate is

$$
\begin{aligned}
\Gamma\left(p, s, s^{\prime}, s^{\prime \prime}\right)= & \int \frac{1}{8(2 \pi)^{2} E_{\mathbf{p} s}} \frac{d^{3} p^{\prime} d^{3} p^{\prime \prime}}{E_{\mathbf{p}^{\prime} s^{\prime}} E_{\mathbf{p}^{\prime \prime} s^{\prime \prime}}}\left|M\left(p, s, p^{\prime}, s^{\prime}, p^{\prime \prime}, s^{\prime \prime}\right)\right|^{2} \\
& \times \delta^{(3)}\left(\mathbf{p}-\mathbf{p}^{\prime}-\mathbf{p}^{\prime \prime}\right) \delta\left(E_{\mathbf{p} s}-E_{\mathbf{p}^{\prime} s^{\prime}}-E_{\mathbf{p}^{\prime \prime} s^{\prime \prime}}\right) .
\end{aligned}
$$

Because of the modifications in the dispersion relations the nature of the integration differs from the simple textbook case. The threshold for the process is derived by determining the momentum at which the matrix element is non-vanishing and the momentum space volume is sufficiently large to ensure that the process is rapid. Due to the modifications in the dispersion relations the momentum space volume differs significantly from the corresponding LLI calculation.

\subsubsection{Kinematic constraints arising from birefringence}

From the form of the dispersion relation (4.1) it is clear that left and right circularly polarized photons travel at different speeds. Linear polarized high energy radiation will be rotated through and energy-dependent angle, depolarizing the radiation. After a distance $d$ the polarization vector of a linearly polarized plane wave with momentum $k$ rotates as [140]

$$
\theta(d)=\frac{\omega_{+}-\omega_{-}}{2} d \simeq \xi \frac{k^{2} d}{2 \kappa} .
$$

Given the finite bandwidth of a detector, $k_{1}<E<k_{2}$, the constraint on the parameter $\xi$ can be derived from the observation of polarization in the relevant bandwidth; if the LV term was large enough there would be no measured net polarization. If some polarization is observed then the angle of rotation across the bandwidth must be less than $\pi / 2[114,142]$. The simple detection of a polarized signal yields the constraint, from (4.4), of

$$
\xi \lesssim \frac{\pi \kappa}{\left(k_{2}^{2}-k_{1}^{2}\right) d}
$$

For a refinement of this argument, and other approaches relying on knowledge of the source, see [163, Section IV.B]. The current best constraint on $|\xi|$ is discussed in Section 4.1.5. An intriguing change in polarization during a GRB was recently reported [263].

\subsubsection{Dynamical constraints arising from photon stability}

With LV photons can decay via pair production, $\gamma \rightarrow e^{+} e^{-}$. The threshold for photon decay is determined as described above, by investigating the rate. Then given the observed stability of high energy photons, constraints can be placed on the new LV terms in the dispersion relations. 
Photon decay generally involves all three LV parameters $\xi, \eta_{+}$, and $\eta_{-}$. However as argued in [141], we can obtain constraints on the pairs of parameters $\left(\eta_{-}, \xi\right)$ or $\left(\eta_{+}, \xi\right)$ by considering the case in which the electron and positron have opposite helicity. We present the calculation for $\eta_{+} \equiv \eta$ and will see that the $\eta_{-}$case is easily obtained from this one. The LV terms are $\eta_{+} p_{-}^{3}$ for the electron and $-\eta_{+} p_{+}^{3}$ for the positron. In the threshold configuration the outgoing momenta are parallel and angular momentum is not conserved. But, above the threshold, the outgoing momenta can deviate from the parallel configuration and, with the additional transverse momentum, the process will conserve angular momentum.

For slight angular deviations from the threshold configuration of the outgoing momenta the matrix element does not vanish and is proportional to the perpendicular momentum of the outgoing particles [141]. The volume of the region of momentum space where photon decay occurs is determined by energy conservation and the boundary of the region occurs when the perpendicular momentum vanishes. We denote the photon momentum $k$, electron momentum $p_{-}$, positron momentum $p_{+}$, and the helicity parameter $\eta=\eta_{+}<0$. Thus, from $\omega=E_{+}+E_{-}$and equations (4.1) and (4.2) we see that

$$
k \sqrt{1 \pm \xi \frac{k}{\kappa}}=p_{+} \sqrt{1+\frac{m^{2}}{p_{+}^{2}}-\eta \frac{p_{+}}{\kappa}}+p_{-} \sqrt{1+\frac{m^{2}}{p_{-}^{2}}+\eta \frac{p_{-}}{\kappa}}
$$

or, using conservation of momentum and the high momentum limit, the expression of energy conservation becomes

$$
\pm \xi \frac{k^{2}}{\kappa} \simeq\left(m^{2}+p_{\perp}^{2}\right)\left(\frac{1}{p_{+}}+\frac{1}{p_{-}}\right)+\frac{\eta}{\kappa}\left(p_{-}^{2}-p_{+}^{2}\right)
$$

where $p_{\perp}$ is outgoing particle transverse momentum. The LV terms in the dispersion relation raise or lower the particle's energy as a function of the momentum. Because of the flattening out of the energy at high momentum for negative $\eta$, the outgoing energy is reduced if one particle carries more momentum than the other. The threshold is thus determined by an asymmetric momentum partition [140]. Partitioning via an asymmetry parameter $\Delta>0$ we have, with $p_{-}=(k / 2)(1-\Delta)$ and $p_{+}=(k / 2)(1+\Delta)$,

$$
\pm \xi \frac{k^{2}}{\kappa}=\frac{4}{k}\left(m^{2}+p_{\perp}^{2}\right) \frac{1}{1-\Delta^{2}}-\frac{\eta k^{2}}{\kappa} \Delta .
$$

This relation is enforced in the rate of equation (4.3) by the energy-conserving delta-function. Carrying out the integrations over the momenta, the integral for the rate is reduced to a single integration over the longitudinal momentum, when the perpendicular momentum is determined by energy conservation. Then

$$
p_{\perp}^{2}= \pm \xi \frac{k^{3}}{4 \kappa}\left(1-\Delta^{2}\right)-m^{2}+\frac{\eta k^{3}}{4 \kappa} \Delta\left(1-\Delta^{2}\right) .
$$

Given the strong constraints on $\xi$ via vacuum birefringence (see Section 4.1.2), $\xi \simeq 0$, we proceed with vanishing $\xi$. The volume in momentum space for photon decay opens up as the perpendicular momentum increases from zero. The threshold is then determined by $p_{\perp}=0$, or

$$
-\frac{4 m^{2} \kappa}{\eta k^{3}}+\Delta\left(1-\Delta^{2}\right)=0
$$

A simple optimization shows that the asymmetry is $\Delta=1 / \sqrt{3}$ and the outgoing momenta are $(k / 2)(1 \pm 1 / \sqrt{3})$ yielding a threshold of

$$
k_{t h}=\left(\frac{6 \sqrt{3} m^{2} \kappa}{\eta}\right)^{1 / 3} \text {, }
$$


as determined from (4.5) with $\xi=0$. The rate increases rapidly above this threshold [141] so observation of photons up to this energy places limits on the size of the parameter $\eta \equiv \eta_{+}$. Thus, when we consider the $80 \mathrm{TeV}$ photons observed by HEGRA [9] we have $\left|\eta_{+}\right|<0.05$. In general high energy photon observations place limits on one helicity. The more general case with non-vanishing $\xi$ may be derived from (4.6), with the complete allowed region in $(\eta, \xi)$ parameter space determined numerically.

It is interesting to note that, contrary to what one might expect from early work $[140,149]$, the threshold for the process is not determined by minimizing the outgoing energy, which is found from the solution of $\left(4 m^{2} \kappa / \eta k^{3}\right) \Delta-\left(1-\Delta^{2}\right)^{2}=0$. Instead we start by asking, does the process occur? The rate (4.3) then shows that the threshold is determined by the opening up of momentum space due to non-vanishing $p_{\perp}$.

There are a wide variety of other processes that can contribute to the dimension 5 LV QED constraints. In addition to the ones discussed above, there is vacuum Cherenkov $\left(\mathrm{e}^{ \pm} \rightarrow e^{ \pm} \gamma\right)$, helicity decay $\left(\mathrm{e}^{ \pm} \rightarrow e^{\mp} \gamma\right.$ ), fermion pair production $\left(e^{ \pm} \rightarrow e^{ \pm} e^{ \pm} e^{\mp}\right)$, photon splitting $(\gamma \rightarrow n \gamma)$, and the shifting of the photon absorption $\gamma \gamma \rightarrow e^{+} e^{-}$threshold. For more on these the reader should consult $[139,141,163]$. In addition the model has been generalized to include processes including dimension 6 CPT even operators in QED and for hadrons [174], and for nuclei [237].

\subsubsection{Neutrino physics}

Planck-scale modifications of neutrino physics are usually considered in the framework of LLI violating effective field theory. It is well-known that neutrinos in a definite flavor state $\left|\nu_{\alpha}\right\rangle$, $\alpha=e, \mu, \tau$, are superpositions of neutrinos in definite mass states, $\left|\nu_{i}\right\rangle, i=1,2,3$,

$$
\left|\nu_{\alpha}\right\rangle=\sum_{i} U_{\alpha i}^{*}\left|\nu_{i}\right\rangle
$$

with the unitary mixing matrix $U_{\alpha i}$. On the other hand, neutrinos in a definite mass state are superpositions of flavor states,

$$
\left|\nu_{i}\right\rangle=\sum_{\beta} U_{i \beta}\left|\nu_{\beta}\right\rangle
$$

In [78] neutrino oscillation in a simplified two-flavor model is considered. The typical oscillation length $L$, i.e. the distance, after which a neutrino, moving approximately at the speed of light in special relativity, is likely to have changed its flavor, is derived in a simple, straight-forward calculation. It depends on the neutrino's energy,

$$
L(E, m)=\frac{2 \pi}{\Delta p} \approx \frac{4 \pi E}{\Delta m^{2}}
$$

with $\Delta m^{2}=m_{2}^{2}-m_{1}^{2}$ and $\Delta p=p_{2}-p_{1}$. The approximate equality is based on $p_{i}=\sqrt{E^{2}-m_{i}^{2}} \simeq$ $E-\frac{m_{i}^{2}}{2 E}$ for each $i=1,2$.

Planck scale modifications arise when we assume modified dispersion relations of the type (leaving aside possible birefringence effects)

$$
E^{2}=p^{2}+m^{2}+\sum_{n} \eta_{i}^{(n)} \frac{p^{n}}{M_{\mathrm{P}}^{n-2}}
$$

with coefficients $\eta_{i}$, possibly different for each mass state. Such MDRs would imply flavor oscillations even for neutrinos with negligible mass, as long as the coefficients $\eta_{i}^{(n)}$ differ from each other, i.e. when Planck-scale effects imply different corrections for different mass states. 
For the sake of simplicity, here we present only the version suggested in [78], based on the (exact) alternative MDR

$$
p^{2}+m^{2}=E^{2}\left[1-\frac{(E-m)^{2}}{M_{\mathrm{P}}^{2}}\right],
$$

which avoids oscillations of neutrinos with zero rest mass. With this, the modified oscillation length becomes

$$
L^{\prime}(E, m)=\frac{2 \pi}{\frac{1}{2 E} \Delta m^{2}-\frac{E^{2}}{M_{\mathrm{P}}^{2}} \Delta m},
$$

with the mass difference $\Delta m=m_{2}-m_{1}$. Neutrino detectors like IceCube may be sensitive to oscillation length corrections of atmospheric neutrinos [195]. For cosmogenic neutrinos, which have oscillated many times on their way to earth, a detection of such effects would be very difficult.

In effective field theory with higher mass dimension terms, coupled to a LLI breaking fourvector and exact energy and momentum conservation, neutrinos are favorite objects for threshold calculations. Neutrino splitting, $\nu \rightarrow \nu \nu \bar{\nu}$ for example, is forbidden in SR, but could be made possible by LV effects. Generally, for processes derived from the MDR (4.7) and energymomentum conservation one obtains a threshold energy $E_{\mathrm{th}} \sim\left(m^{2} M_{\mathrm{P}}^{n-2}\right)^{\frac{1}{n}}$. With $\eta_{i}$ assumed to be of order unity, the small neutrino mass leads to a threshold energy of $\sim 20 \mathrm{TeV}$ for $\nu$ splitting. If this process takes place, it must result in a cutoff in the energy spectrum of cosmic neutrinos. Taking the decay length for neutrinos above the threshold energy into account, for $\eta_{i} \sim 1$ in [195] an estimate of $10^{18}-10^{19} \mathrm{eV}$ for the cutoff is given, when the neutrinos come from a distance of the order of Mpc. Observation of higher neutrino energies would lower the upper bounds to the parameters $\eta_{i}^{(n)}$.

\subsubsection{Current bounds from astrophysical observation: summary}

The current best constraints on the dimension 5 LV QED parameters arise from $\gamma$-ray bursts and from an extensive analysis of the spectrum from the Crab Nebula [172]. Using the vacuum birefringence effect and recent observations of the $\gamma$-ray burst GRB041219a, the dimension 5 photon parameter $\xi$ has been constrained $|\xi| \lesssim 10^{-14}[161,252]$. At 95 confidence level $\left|\eta_{ \pm}\right|<10^{-5}$ from the analysis of the spectrum from the Crab nebula [173]. Since the terms are already suppressed by the Planck scale, terms arising from dimension 5 operators are sufficiently constrained as to appear ruled out. The current constraints, and some prospects for further improvements are illustrated in Fig. 1.

Other astrophysical arenas have been suggested for testing these theories including neutron stars, $[74,118]$. But it is clear from these studies that in the EFT framework the effects are too small to be observationally accessible $[118]^{10}$.

Considering that the Planck scale is already factored into the modifications, the severity of the constraints is impressive. These results from astrophysics inform the development of quantum gravity. However, there is another reason to suspect that these modifications do not occur in the EFT framework, the naturalness problem.

\subsubsection{The naturalness problem}

Within the EFT framework a naturalness problem suggests that the LV operators are ruled out. The argument is based on renormalization: The higher dimension LV operators generate

\footnotetext{
${ }^{10}$ The result does not immediately extend to the DSR context $[25,118]$. In the DSR context there are no new thresholds and the usual special relativity thresholds are only slightly modified [128].
} 


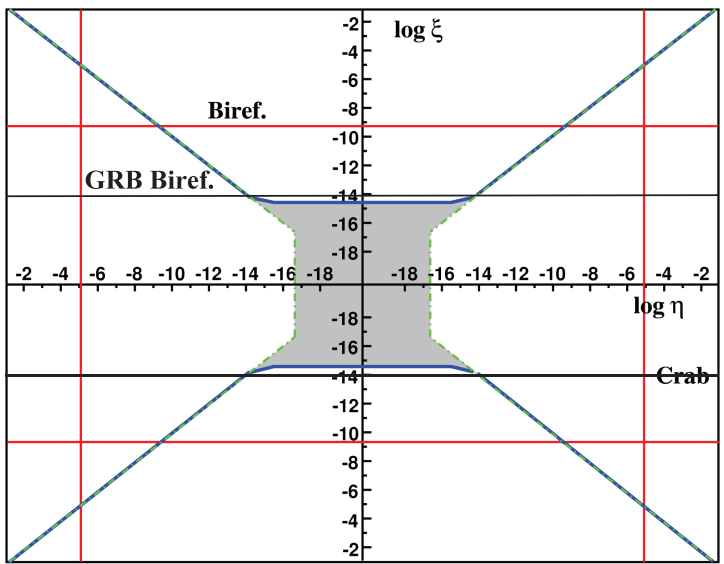

(a)

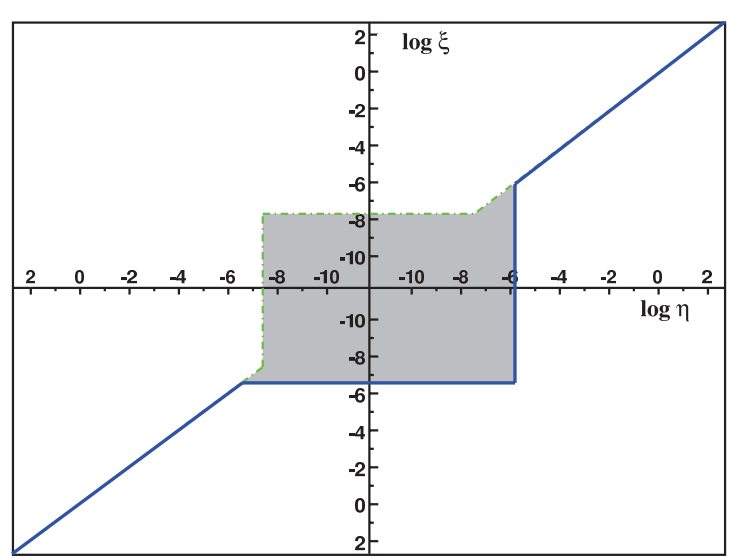

(b)

Figure 1. The constraints on dimension 5 (a) and dimension 6 (b) QED parameters. The log-log plots show the allowed region (dark grey) and the constraints. The current constraints on dimension 5 parameters are shown in red and grey. The grey horizontal lines are due to constraints due to the lack of birefringence (see Section 4.1.2) from GRB photons [161,252]. The red vertical lines are due to the analysis of the Crab spectrum [172]. The blue solid lines show limits that would arise from an upper threshold on pair production at $k_{\mathrm{th}} \simeq 10^{20} \mathrm{eV}$. The green dash-dot lines show the constraints that would arise from a $\gamma$-decay threshold of $k_{\mathrm{th}} \simeq 10^{19} \mathrm{eV}$. The dimension 6 constraints (b) have the same color coding. The GRB birefringence constraint is not relevant to the dimension 6 case since the operator is parity even, contrary to what is reported in [161]. Original plots courtesy of Stefano Liberati. See [163] for more detail.

lower dimension operators through radiative corrections and the low-energy effective theory will contain all terms consistent with the symmetries of the microscopic theory. The usual power-counting arguments that give divergences also determines the natural size of the Planckscale suppressed terms. Generically these appear at dimension 4 or less, with no Planck scale suppression. One can show $[80,81]$ that the operators generated by radiative corrections produce effects that are incompatible with current particle data. Thus the LV parameters would have to be unnaturally fine-tuned to cloak the LV.

Within the EFT framework this naturalness problem is significant. However if there is a custodial symmetry then the radiative terms will not appear at lower dimension. Even a subgroup of the symmetry manifest in the macroscopic, continuum approximation is sufficient. A good example of this is in Euclidean lattice theory where the discrete rotation sub-group on a hypercubic lattice, which becomes the full rotation group as the lattice spacing goes to zero, is enough to prevent the generation of the lower dimensional operators. Other symmetries existing in the vast range of scales from the "low" TeV scale to the high Planck scale might protect the theory from these terms. This is the case for a model with supersymmetry; the symmetry-preserving operators first appear at dimension 5 [120]. This solves the naturalness problem in that the lower dimensional operators are protected by a custodial symmetry. But, of course, supersymmetry is not a low-energy symmetry. When supersymmetry is softly broken with explicit symmetry breaking terms at a scale $M_{\text {SUSYB }}$, the lower dimension terms return, albeit additionally suppressed by factors of $M_{\mathrm{SUSYB}} / \kappa$ raised to some power so, again, the naturalness problem returns at dimension 5 and the parameters would have to be fine-tuned. At dimension 6 however, the additional suppression is sufficient to evade current limits, provided the supersymmetry breaking scale $M_{\text {SUSYB }}<100 \mathrm{TeV}$ [63].

Another possibility was raised recently. Gambini et al. [103] study a Euclidean lattice model, with distinct spatial, $a$, and temporal, $(1+a \mu) a$ lattice scales. The authors find that at the one 
loop order the leading contribution to effective dimension 4 operators is $a \mu$. If one takes the stand that the lattice spacing $a$ should remain small but finite then the lower dimension term in the selfenergy is suppressed by the additional factor $\mu$ and thus could evade the current bounds on this effect. Nonetheless within the EFT framework of LV this does not solve the naturalness problem since the theory would have to be fine-tuned through the scale $\mu$. See [216] for further comments.

Incidentally Gambini et al. show that in the 4D "isotropic scaling" case in which the temporal lattice scaling is also $a$, that lower dimensional corrections do not arise, since the "isotropy" symmetry protects the theory from such corrections. The authors argue in Section IV.B of [102] that in a generally covariant theory the propagators should be constructed with reference matter fields, physical "rods and clocks" rather than the flat background used in EFT. Since the distribution associated to these physical rods and clocks cannot be infinitely narrow, and should be considerably larger than the Planck scale, they would provide a natural cutoff for LV effects without the generation of corrections at lower dimension. In the EFT framework, the widths could provide an additional scale and associated additional suppression, as in the soft breaking of super symmetry. It would be interesting to see whether the hint of just such a scale and suppression in the modified dispersion relations (3.3) could be made more precise.

\subsubsection{Generalizations and prospects for improved constraints}

Given the naturalness problem and the tight constraints on dimension 5 LV QED in the EFT framework several generalizations have been considered. Building on earlier work dimension 6 CPT-even LV operators were studied [193] and constrained [163]. In the pure photon sector in the Standard Model Extension there are bounds up to dimension 9 [151].

Ultra-high energy cosmic rays (UHECR) hold promise for tightening the current constraints. One striking aspect of the cosmic ray spectrum is the expected GZK cutoff. In the LLI theory cosmologically sourced ultra-high energy protons interact with cosmic microwave background producing pions and lower energy protons, neutrinos, and $\gamma$-rays, reducing the energy of the original photons. The resulting GZK (so named for Greisen, Zatsepin and Kuzmin) cutoff occurs at $E_{\mathrm{GZK}} \simeq 5 \times 10^{19}\left(\omega_{\mathrm{cmb}} / 1.3 \mathrm{meV}\right)^{-1} \mathrm{eV}$, where $\omega_{c m b}$ is the energy of the background photon. LV terms in the hadronic dispersion relations can shift the threshold for photo-pion production $[149,174]$. Recently observations in support of the GZK cutoff were announced $[1,4$, 221] so the LLI theory is favored. A detailed statistical analysis of UHECRs severely constrains proton and pion parameters in the dimension 5 and 6 CPT-even model [174].

The GZK process provides another mechanism for constraints. Photo-pion production from the UHECR protons leads to the production of $\gamma$-ray pairs. Cosmic ray observatories have placed limits on the fraction of UHE photons in the cosmic ray spectrum [5,231] that can in turn place limits on photon parameters. With LV, processes can have upper thresholds at high energies and the existence of an upper threshold for pair production at high energies increases the photon fraction, yielding a conflict with current photon fraction bounds [100]. Likewise a neutrino flux is created by pions originating from the same photo-pion production. The possible tests of LV in neutrino physics are outlined in $[163,195]$.

Additionally, in an intriguing development in the non-relativistic regime Amelino-Camelia et al. argue that cold-atom-recoil experiments constrain dispersion relations modifications of the form $m p / \kappa[24]$.

There remains work to be done on the theoretical side, understanding the status of LLI in LQG and, if verified, constraining the MDR parameters, for instance as in equations (3.2) and (3.3). In particular, it would be very interesting to determine which specific structures in LQG, together with any additional assumptions, yield specific effects. As those specific effects

are constrained, then the structures and assumptions may be tested directly. Some questions for further work include: 
- Since the scaling in terms of $\Upsilon$ holds for different dimension operators, the clock comparison experiments, for instance, should yield bounds on $\Upsilon$ and vacuum birefringence for dimension 4 (see [141] page 29). The correlation between the dimension 3 and 4 results could yield limits on $\Upsilon$.

- Building on $[233,234]$ is there non-integer scaling in the dispersion relations? What specific structures in LQG is the scaling tied to?

- Is there an analogous calculation that can be completed in the spin foam context?

- Is is not easy to see how such non-integer scaling could occur in EFT, can it be ruled out? Or, is EFT missing something? (see, e.g. [102,103]).

- Can physical measurements and the underlying spatial discreteness of LQG kinematics be reconciled with the EFT framework?

\subsection{EFT Phenomenology without Lorentz violation: a combinatoric lever arm}

Another model in the EFT framework demonstrated that there may be "lever arms" intrinsic to the granularity of space $[189,190]$, demonstrating one way in which the combinatorics of SWN nodes may raise the effective scale of the granularity. The model is based on the angle operator.

The asymmetry in the angular spectrum shifts the distribution of angles away from the $\sin \theta$ isotropic distribution of polar angles in 3-dimensional flat space. The distribution is recovered when the spin through the three surfaces, "flux" or $s_{i}$, satisfies $1 \ll s_{i} \ll s_{3}$ for $i=1,2$. Fluxes $\vec{s}$ that satisfy these relations are called "semi-classical". These fluxes are essentially the areas of the partitions discussed in Section 2.4.

The model of [190] is based on the assumption that the states of the atom of 3-geometry or intertwiner are equally likely. In addition to the uniform probability measure, the model assumes that all incident edges to the node are all spin- $\frac{1}{2}$. The combinatorics of the atom can be solved analytically for semi-classical fluxes. Given the fluxes $\vec{s}$, or areas of the partitions, and the labels $\vec{j}$ on the intertwiner vertex, or "intertwiner core", that connects the three partitions, the normalized probability distribution is given $(\vec{n}=2 \vec{j})$

$$
p_{\vec{s}}(\vec{n}) \simeq \prod_{i=1}^{3} \frac{n_{i}}{s_{i}} \exp \left(-\frac{n_{i}^{2}}{2 s_{i}}\right) .
$$

Accessible measurements of the atom include 3-volume, which is approximately determined by the total area or flux, and angle, determined by the states $|\vec{n}\rangle$ of the intertwiner core. The fluxes $\vec{s}$ determine a mixed state,

$$
\rho_{\vec{s}}=\sum_{\vec{n}} p_{\vec{s}}(\vec{n}) P_{|\vec{n}\rangle},
$$

where $P_{|\vec{n}\rangle}$ is the projector on the orthonormal basis of the intertwiner core. The sum is over the admissible 3 -tuples of integers $\vec{n}$ such that $n_{i} \leq s_{i}$. In the discrete case the projector is $P_{|\vec{n}\rangle}=\left|\theta_{I}\right\rangle\left\langle\theta_{I}\right|$, where $\left|\theta_{I}\right\rangle=\sum_{\vec{n}} c_{\theta_{I}}(\vec{n})|\vec{n}\rangle$. The probability of finding the angle eigenvalue $\theta_{I}$ in the mixed state $\rho_{\vec{s}}$ is

$$
\operatorname{Prob}\left(\theta=\theta_{I} ; \rho_{\vec{s}}\right)=\operatorname{tr}\left(\rho_{\vec{s}} P_{\theta_{I}}\right)=\sum_{\vec{n}} p_{\vec{s}}(\vec{n})\left|\left\langle n \mid \theta_{I}\right\rangle\right|^{2} \equiv p_{\vec{s}}(\theta) .
$$

This procedure can be used to calculate the probability in the continuum approximation [190]

$$
P_{\vec{s}}(\theta)=\int \mathrm{d}^{3} n p_{\vec{s}}(\vec{n})\left|c_{\theta}(\vec{n})\right|^{2} \delta(\theta-\theta(n)) .
$$


The integration of equation (4.8) is straightforward [190]. The key step in the calculation is the identification of the "shape parameter" $\epsilon:=\sqrt{s_{1} s_{2}} / s_{3}$ that measures the asymmetry in the distribution of angles. The resulting measure, when expressed in terms of Legendre polynomials and to $O\left(\epsilon^{3}\right)$, is [190]

$$
\rho_{\epsilon}(\theta) \simeq \sin \theta\left(1-\frac{8}{\pi} P_{1}(\cos \theta) \epsilon+\frac{3}{2} P_{2}(\cos \theta) \epsilon^{2}\right) .
$$

The affect of the modified distribution of polar angles is that the 'shape' of space is altered by the combinatorics of the vertex; the local angular geometry differs from flat 3-space. While these effects would be in principle observable at any flux, the results here are valid for semi-classical flux, $1 \ll s_{j} \ll s_{3}$ for $j=1,2$. As mentioned briefly above, the total flux $s=\sum_{i} s_{i}$ determines the 3 -volume of the spatial atom and thus an effective mesoscopic length scale, $\ell_{s}=\sqrt{s} / \kappa$, greater than the fundamental discreteness scale of $1 / \kappa$. The combinatorics of the intertwiner provides a lever arm to lift the fundamental scale of the quantum geometry up to this larger mesoscopic scale. So while the the shape parameter $\epsilon$ is free from the Planck scale, the effective length scale, determined by total flux $s$, is tied to the discreteness scale of the theory.

If the scale $\ell_{s}$ of the spatial atom is large enough then the underlying geometry would be accessible to observations of particle scattering. An overly simple example of Bhabha scattering is worked out in [190]. However, the model needs further development. In particular for coherent states defined with classical directions lever arm is too short to raise the scale up to an observable window [189]. It is an open question as to whether the long combinatoric lever arm exists for coherent states built from quantum information intrinsic to the states of the atom of geometry. Further, the QED vertex should be modeled in detail.

\subsection{Non-commutative field theory}

\subsubsection{Overview}

As discussed in Section 3.7, a non-commutative geometry can be generated when the coordinates functions $X_{\mu}$ are operators that do not commute. To introduce a field over this non-commutative space, we have to consider a field over these coordinates operators. As in Section 3.7, we shall focus on flat non-commutative geometries. In the case of a scalar field, this can be done rigorously in most of the non-commutative geometries discussed in Section 3.7.

However, instead of using operators and all their machinery, we can simplify the mathematics and use instead c-numbers $x_{\mu}$ for the coordinates with a non-trivial product which encodes the non-commutative structure. This approach can be understood either as a specific representation of the non-commutative algebra (defined in terms of operators) or as an example of deformation. At the end, the formalism is equivalent. The representation is obtained through the Weyl map $W$, which is such that

$$
W\left(X_{\mu}\right)=x_{\mu}, \quad W\left(X_{\mu} X_{\nu}\right)=x_{\mu} * x_{\nu} .
$$

We see that the non-trivial operator product is encoded in a new product denoted $*$. This is naturally extended to general (analytic) functions. The deformation can be understood as modifying the point-wise product of functions. The usual algebra $\mathcal{C}^{\infty}\left(\mathbb{R}^{4}\right)$ of commutative functions over spacetime $\mathbb{R}^{4}$ is equipped with the pointwise product

$$
\left(f_{1} \cdot f_{2}\right)(x)=f_{1}(x) f_{2}(x), \quad \forall f_{i} \in \mathcal{C}^{\infty}\left(\mathbb{R}^{4}\right) .
$$

It becomes a non-commutative algebra if we replace the pointwise product by the $*$-product.

$$
\left(f_{1} \cdot f_{2}\right)(x) \rightarrow\left(f_{1} * f_{2}\right)(x) .
$$


The $*$-product of coordinates functions $x_{\mu}$ encodes a similar non-commutative structure as the operators discussed in Section 3.7. For example, respectively in the Moyal case and the $\kappa$ Minkowski case, we will have

$$
\begin{aligned}
& \text { Moyal space: } \quad x_{\mu} * x_{\nu}-x_{\nu} * x_{\mu}=\frac{i}{\kappa^{2}} \theta_{\mu \nu} \\
& \kappa \text {-Minkowski space: } \quad x_{0} * x_{i}-x_{i} * x_{0}=\frac{1}{\kappa} x_{i}, \quad x_{j} * x_{i}-x_{i} * x_{j}=0 .
\end{aligned}
$$

This representation is not unique. For example for the Moyal spacetime, the most popular representations of the $*$-product are given by [42]

$$
\begin{array}{ll}
\text { Moyal: } & \left(f_{1} *_{M} f_{2}\right)(x)=f_{1}(x) e^{\frac{i}{2} \theta^{\mu \nu} \overleftarrow{\partial_{\mu}} \overrightarrow{\partial_{\nu}}} f_{2}(x), \\
\text { Voros: } & \left(f_{1} *_{V} f_{2}\right)(x)=f_{1}(x) e^{\frac{i}{2}\left(\theta^{\mu \nu} \overleftarrow{\partial_{\mu}} \overrightarrow{\partial_{\nu}}-\overleftarrow{\partial^{\mu}} \overrightarrow{\partial_{\mu}}\right)} f_{2}(x)
\end{array}
$$

This *-product construction of a non-commutative space is analogue to the phase-space formulation of quantum mechanics introduced by Moyal [207] and Groenewald [119].

With the help of this $*$-product, we can generalize the usual construction of the scalar field action with a $\phi^{3}$ interaction term for a real scalar field $\phi$ from

$$
\begin{aligned}
& \int\left[d^{4} x\right]\left[\left(\left(\partial_{\mu} \phi\right) \cdot\left(\partial^{\mu} \phi\right)\right)(x)+m^{2}(\phi . \phi)(x)+\frac{\lambda}{3 !}(\phi . \phi . \phi)(x)\right] \text { to } \\
& \int\left[d^{4} x\right]\left[\left(\left(\partial_{\mu} \phi\right) *\left(\partial^{\mu} \phi\right)\right)(x)+m^{2}(\phi * \phi)(x)+\frac{\lambda}{3 !}(\phi * \phi * \phi)(x)\right] .
\end{aligned}
$$

Note that in the case of the DFR spacetime as well as Snyder spacetime interpreted as a subspace of a larger space, one should also consider the measure on the extra coordinates. We refer to $[88,111]$ for further details on these cases.

The usual approach in QG phenomenology is to construct a scalar field theory as it is the simplest field theory. The case of fields with higher spin is more complicated. Indeed, spin is usually related to a representation of the Poincaré group. If we deform this symmetry group to accommodate for the non-commutative structure, the representation theory will change and hence what we call spin could change. Furthermore the relation spin-statistics will be nontrivial since, in the case of a quantum group, the tensor product of representations becomes non-commutative. See [44,214] for the Moyal case and [30,264] for the $\kappa$-Minkowski case. At this stage, it is only in the Moyal case where the notions of spinor [64] and vector field have been introduced, since the non-commutative structure is a "mild" one in terms of deformation (it is a twist [182]).

Since the hope is to measure some semi-classical QG effects in the propagation of electromagnetic field, one would like to construct a U(1) gauge theory in one's preferred non-commutative spacetime. Unfortunately this can be done to our knowledge only in the Moyal case. Even in this case, things are highly non trivial at the classical level (and of course at the quantum case as well!). For example, a U(1) gauge theory behaves essentially like a non-Abelian gauge theory due to the non commutativity [240]. It is difficult to construct non-Abelian gauge theories with simple local groups since the non-commutativity will always destroy the traceless property [144]. There are also constraints on the transformations of the matter fields under gauge transformations [77]. Since the dispersion relation is not modified in the Moyal case, we do not expect to see effects measurable in gamma-ray bursts a priori.

There is no definite construction of an Abelian nor non-Abelian Yang-Mills theory in either of the $\kappa$-Minkowski, DFR and Snyder cases. This is an important issue to address if one intends to make precise predictions for the FERMI experiment. 
Once we have defined the scalar field action in spacetime, we can try to define it in momentum space, that is we introduce plane-waves and a Fourier transform. Majid has introduced in a general setting the notion of Fourier transform for Hopf algebras [182]. We follow here a more pedestrian presentation.

As we have emphasized in Section 3.1, momentum space is equipped with a group structure since we need to add momenta. Furthermore the pointwise product of plane-waves as functions on spacetime incorporates this momenta addition,

$$
\left(e_{p} \cdot e_{q}\right)(x)=e^{p \cdot x} e^{i q \cdot x}=e^{i(p+q) \cdot x}=e_{p+q}(x) .
$$

The generalization to the non-commutative case then uses the $*$-product between plane-waves. The Moyal case and the $\kappa$-Minkowski case lead to different cases.

$$
\begin{aligned}
& \text { Moyal: } \quad\left(e_{p} * e_{q}\right)(x)=e^{i(p+q) \cdot x} e^{\frac{i}{2} p \cdot \theta \cdot q}, \\
& \kappa \text {-Minkowski: } \quad\left(e_{p} * e_{q}\right)(x)=e_{p \oplus q}(x),
\end{aligned}
$$

where we have used the non-trivial sum $p \oplus q$ of (3.15) inherited from the non-Abelian group $\mathrm{AN}_{3}$ as discussed in Section 3.7. With this in hand, we define the Fourier transform and its inverse as

$$
\hat{\phi}(p)=\mathcal{F}(\phi)(p) \equiv \int\left[d^{4} x\right]\left(\phi * e_{p}\right)(x), \quad \phi(x)=\mathcal{F}^{-1}(\hat{\phi})(x) \equiv \int\left[d^{4} p\right] \hat{\phi}(p) e_{x}(\ominus p),
$$

where we have used the relevant measure $\left[d^{4} p\right]$ on momentum space. For example, $\mathrm{AN}_{3}$ is isomorphic to half of de Sitter space, hence $\left[d^{4} p\right]$ will be the measure on de Sitter space expressed in the chosen coordinates. In the case of the inverse Fourier transform $\mathcal{F}^{-1}$, we see the planewave as a function over momentum space and we have to deal with the relevant inverse $\ominus$ of the addition $\oplus$, i.e. $\ominus p \oplus p=0=p \oplus(\ominus p)$. We use the commutative pointwise product for the algebra of functions over the momentum manifold. Deforming this product as well, i.e. to have a non-commutative momentum space, would mean that we are dealing with a quantum group momentum space. This case has not yet been studied to our knowledge.

To perform the Fourier transform of the action (4.9), it is usually convenient to consider the plane-wave as the eigenfunction of the derivative $\partial_{\mu}$. This requires in general a careful study of the differential calculus over the non-commutative space as for example in $\kappa$-Minkowski case. We refer to $[96,241]$ for further details on this. We assume that the plane wave is the eigenfunction of the derivative so that

$$
\partial_{\mu} e_{p}(x)=i p_{\mu} e_{p}(x), \quad \mathcal{F}\left(\partial_{\mu} \phi\right)(p)=i p_{\mu} \hat{\phi}(p) .
$$

Note that when dealing with functions over a group (which we call here momentum space), there is another notion of Fourier transform which is usually used. For example, in the case of compact groups (for instance $\mathrm{SU}(2)$ as a $3 \mathrm{~d}$ Euclidian momentum space), the Fourier transform one would think to use consists in decomposing the functions over the group in terms of the matrix elements of the representations of the group, thanks to the Peter-Weyl theorem. This is not the Fourier transform we have discussed above in (4.10). There exists nevertheless a natural isomorphism between the different types of Fourier transform [143]. To our knowledge the isomorphism has not been studied in detail in the case of non-compact groups for momentum space.

With the Fourier transform (4.10) the $\lambda \phi^{3}$ action becomes, in the Moyal case,

$$
\int[d p][d q] \hat{\phi}(p)\left(p^{2}-m^{2}\right) \hat{\phi}(q) \delta(p+q)
$$




$$
-\frac{\lambda}{3 !} \int[d p]^{3} \hat{\phi}\left(p_{1}\right) \hat{\phi}\left(p_{2}\right) \hat{\phi}\left(p_{3}\right) e^{i\left(p_{1} \cdot \theta \cdot p_{2}+\left(p_{1}+p_{2}\right) \cdot \theta \cdot p_{3}\right)} \delta\left(p_{1}+p_{2}+p_{3}\right),
$$

and, in the $\kappa$-Minkowski case,

$$
\int[d p][d q] \hat{\phi}(p) \mathcal{K}(p) \hat{\phi}(q) \delta(p \oplus q)-\frac{\lambda}{3 !} \int[d p]^{3} \hat{\phi}\left(p_{1}\right) \hat{\phi}\left(p_{2}\right) \hat{\phi}\left(p_{3}\right) \delta\left(p_{1} \oplus p_{2} \oplus p_{3}\right) .
$$

Note that we still have a conservation of momenta in both cases. We notice the key difference: In the Moyal case, the Dirac delta function comes decorated with a phase depending on momentum and $\theta$, but the conservation of momenta is obtained through the usual commutative addition of momenta. In the $\kappa$-Minkowski case, the conservation of momenta is done through the modified addition, inherent to the new group structure that we use. Furthermore we could have a modified propagator $\mathcal{K}(p)$ - related to a modified dispersion relation - in the $\kappa$-Minkowski case.

If we focus on a general group for momentum space (therefore on a spacetime with Lie algebra type), we can rewrite the action for a scalar field in momentum space just in terms of group elements

$$
\int[d g]^{2} \hat{\phi}\left(g_{1}\right) \mathcal{K}\left(g_{1}\right) \hat{\phi}\left(g_{2}\right) \delta\left(g_{1} g_{2}\right)-\frac{\lambda}{3 !} \int[d g]^{3} \hat{\phi}\left(g_{1}\right) \hat{\phi}\left(g_{2}\right) \hat{\phi}\left(g_{3}\right) \delta\left(g_{1} g_{2} g_{3}\right) .
$$

$[d g]$ is the Haar measure on the group of interest. Note that to be rigorous one should be careful with the different coordinates patches used to cover the group. We omit this subtlety and refer to $[95,143]$ for further details. Equation (4.11) is nothing but a sum of convolution products of functions over the group evaluated at the identity since

$$
\begin{aligned}
& (\hat{\psi} \circ \hat{\phi})(g)=\int[d g]^{2} \hat{\phi}\left(g_{1}\right) \mathcal{K}\left(g_{1}\right) \hat{\phi}\left(g_{2}\right) \delta\left(g_{1} g_{2} g^{-1}\right) \quad \text { with } \quad \hat{\psi}\left(g_{1}\right)=\hat{\phi}\left(g_{1}\right) \mathcal{K}\left(g_{1}\right), \\
& (\hat{\phi} \circ \hat{\phi} \circ \hat{\phi})(g)=\int[d g]^{3} \hat{\phi}\left(g_{1}\right) \hat{\phi}\left(g_{2}\right) \hat{\phi}\left(g_{3}\right) \delta\left(g_{1} g_{2} g_{3} g^{-1}\right) .
\end{aligned}
$$

The usual commutative scalar field theory can also be put under this form if we use the Abelian group $\mathbb{R}^{4}$. The $\kappa$-Minkowski case appears when the group is non-Abelian and is $\mathrm{AN}_{3}$.

Deforming the addition of momenta can be seen as another way to deform our theory. We can start with the standard scalar field theory defined over momentum space given by $\mathbb{R}^{4}$ and introduce a non-trivial addition. By considering plane-waves with a non-trivial product, inherited from the deformed momenta addition, we reconstruct the $*$-product. This way of proceeding is essentially the dual to the one we have presented here.

The abstract writing of the scalar field action is useful as it can help to understand how matter can arise from a spinfoam [112]. We shall discuss this in the next subsection.

We have discussed the classical definition of the scalar field action. Before discussing the quantum case, let us comment on two specific topics: symmetries and conserved charges. We have discussed in Section 3.7 how some non-commutative spaces can be seen as flat noncommutative spaces. We can therefore expect that the action of the scalar field will be invariant under the deformed Poincaré symmetries [76,95]. A scalar field will be simply the trivial representation of the deformed Poincaré group. According to the chosen deformation, the main difference with the usual case will be how the tensor product of fields are transformed. For example, for a translation by $\epsilon$ in the $\kappa$-Minkowski case, we have

$$
\hat{\phi}(p) \rightarrow e_{\epsilon}(p) \hat{\phi}(p), \quad \hat{\phi}(p) \otimes \hat{\phi}(q) \rightarrow e_{p \oplus q}(\epsilon)(\hat{\phi}(p) \otimes \hat{\phi}(q))=\left(e_{p} * e_{q}\right)(\epsilon)(\hat{\phi}(p) \otimes \hat{\phi}(q)) .
$$

We notice the appearance of the $*$-product and the modified momenta addition which encodes the deformation of the symmetries. In particular $\hat{\phi}(p) \otimes \hat{\phi}(q)$ and $\hat{\phi}(q) \otimes \hat{\phi}(p)$ do not transform in the same way since $p \oplus q \neq q \oplus p$. This is another way to see that the tensor product is 
no longer commutative when using quantum groups. Nevertheless, it can be shown that the scalar field action in the different non-commutative spacetimes is invariant under the relevant deformed symmetries as expected. In the $\kappa$-Minkowski case, we have a deformation of the Poincaré symmetries but one can also encounter Lorentz symmetry breaking if one is not careful about the choice of coordinates patch [95].

If we have symmetries, one can expect to have conserved charges following Noether's theorem. This is indeed true in the non-commutative context. They have been analyzed for both Moyal [20] and $\kappa$-Minkowski spacetimes [96]. The analysis relies on the understanding of the differential calculus over the non-commutative space. For example in the $\kappa$-Minkowski case there exist different types of differential calculus [241] which leads then to different notions of conserved charges $[6,96]$. We refer to the original articles for more detail.

The quantization of non-commutative scalar field theory can be performed. The Moyal case has been analyzed in great detail, the other non-commutative geometries much less so. For a recent overview of some phenomenology of field theory in Moyal spacetime, we refer to [43].

As we have alluded few times already, when dealing with a quantum group, the tensor product of its representations (i.e. here the scalar field) becomes non-commutative. If we want to permute representations we have to use a structure called "braiding", which encodes the non-commutativity of the tensor product. Now, when constructing Feynman diagrams we use Wick's theorem and fields permutations extensively. Then we have a choice: either consider the braiding related to the deformation of the Poincaré group, or use a trivial braiding (i.e. the one associated to the usual Poincaré group). In the first case, this will ensure that the Feynman diagrams are invariant under the deformed Poincaré group. This is the setting of braided field theory as developed by Oeckl [209] and Majid [182]. In the second case, we can encounter symmetry breaking. In particular non-planar diagrams will often fail to be invariant under the Poincaré symmetries. Quite strikingly, it has been shown that such braiding in the Moyal case means that the non-commutative scalar field theory has the same amplitude as the commutative one $[45,92]$ ! Thus we see that the Moyal deformation is a "mild" one. Quantum gauge theories do feel the non-commutativity since at the classical level already - as we have recalled - some non-trivial effects happen.

From this perspective, not considering the braiding in the Moyal case leads to a more interesting scalar field theory, not equivalent to the standard one. However one has to face a new problem: the ultraviolet-infrared (UV-IR) mixing. This problem arises when dealing wit non-planar diagrams which are most sensitive to the non-commutative structure as we recalled above. When the external momenta are not zero, the space-time non-commutativity regularizes the ultraviolet divergences, just like one would hope non-commutativity to do. However when the external momenta are zero, the amplitude of the non-planar diagram diverges again. Small (external) momenta lead to a high energy divergence [196,205]. This has made the control of the renormalization analysis of the Moyal non-commutative scalar field theory quite involved $[122,126]$.

One might wonder if braiding could simplify the amplitudes of a quantum scalar field theory in the $\kappa$-Minkowski case. A priori, we should not expect to recover the same amplitudes as in the un-deformed case there one really modifies momentum space by adding curvature. In particular the measure is different in the flat case and curved case. This is only a preliminary argument since the $\kappa$-Minkowski case faces another issue: the braiding is not completely understood; see [265] for further comments. Finally, the IR-UV mixing also arises when considering a scalar field in $\kappa$-Minkowski [121].

As a final comment, let us recall that non-commutative geometry was introduced by Snyder [250] as a hope to regularize the divergencies of field theory. This hope was not realized in general. Perhaps the only examples where this holds are DFR space [41] and, when momentum space is a quantum group [183,220]. 


\subsubsection{Relating non-commutative field theory and spinfoam models}

Group field theories (GFT) are tools to generate spinfoams. Namely, they are scalar field theories with a non-local interaction term, built on a product of groups. Upon quantization, using the path integral formalism, the Feynman diagram amplitudes can be interpreted as spinfoam amplitudes, constructed out of gravity or some topological models. As we have recalled in Section 4.3.1, even standard field theories can seen as some kind of group field theories. The only difference is whether the group is Abelian or not, so that the dual space becomes commutative or not. Realizing this is the first key to understand how one can recover non-commutative field theories encoding matter from a spinfoam model.

GFT built on $\mathrm{SO}(4,1)$, it could probably contain in some ways a DSR scalar field theory. One has to carefully identifies the scalar degrees of freedom in the spinfoam GFT. One key-difficulty is to identify the DSR propagator since often the spinfoam GFT has a trivial propagator.

As always, 3d quantum gravity being simpler than 4d quantum gravity models, it provides the ideal framework to illustrate this idea. We consider therefore Boulatov's GFT which generates the Ponzano-Regge spinfoam amplitude describing Euclidian 3d quantum gravity [65]. It is defined in terms of a real scalar field $\varphi: \mathrm{SU}(2)^{3} \rightarrow \mathbb{R}$, which is required to be gauge invariant under the diagonal right action of $\mathrm{SU}(2)$,

$$
\varphi\left(g_{1}, g_{2}, g_{3}\right)=\varphi\left(g_{1} g, g_{2} g, g_{3} g\right), \quad \forall g \in \mathrm{SU}(2) \text {. }
$$

Boulatov's action involves a trivial propagator and the tetrahedral interaction vertex,

$$
\begin{aligned}
S_{3 d}[\varphi]= & \frac{1}{2} \int[d g]^{3} \varphi\left(g_{1}, g_{2}, g_{3}\right) \varphi\left(g_{3}, g_{2}, g_{1}\right) \\
& -\frac{\lambda}{4 !} \int[d g]^{6} \varphi\left(g_{1}, g_{2}, g_{3}\right) \varphi\left(g_{3}, g_{4}, g_{5}\right) \varphi\left(g_{5}, g_{2}, g_{6}\right) \phi\left(g_{6}, g_{4}, g_{1}\right) .
\end{aligned}
$$

We note that the interaction term is defined over six copies of $\mathrm{SU}(2)$, the action is therefore very non-local, even in the non-commutative sense. The equation of motion is

$$
\varphi\left(g_{3}, g_{2}, g_{1}\right)=\frac{\lambda}{3 !} \int \mathrm{d} g_{4} \mathrm{~d} g_{5} \mathrm{~d} g_{6} \varphi\left(g_{3}, g_{4}, g_{5}\right) \varphi\left(g_{5}, g_{2}, g_{6}\right) \varphi\left(g_{6}, g_{4}, g_{1}\right) .
$$

In his $\mathrm{PhD}$ thesis [166], Livine identified some solutions of this equation of motion.

$$
\varphi^{(0)}\left(g_{1}, g_{2}, g_{3}\right)=\sqrt{\frac{3 !}{\lambda}} \int \mathrm{d} g \delta\left(g_{1} g\right) F\left(g_{2} g\right) \delta\left(g_{3} g\right), \quad F: G \rightarrow \mathbb{R},
$$

when $\int[d g] F(g)^{2}=1$ (or $F=0$ ).

Later on, Fairbairn and Livine realized that the scalar perturbations $\hat{\phi}(g)$ around some specific solutions would actually behave exactly like a scalar field theory with SU(2) as momentum space [90]. The effective action for $\hat{\phi}$ is constructed using $\varphi\left(g_{1}, g_{2}, g_{3}\right)=\varphi^{(0)}\left(g_{1}, g_{2}, g_{3}\right)+$ $\hat{\phi}\left(g_{1} g_{3}^{-1}\right)$ and Boulatov's action (4.12)

$$
\begin{aligned}
S_{\text {eff }}[\hat{\phi}]= & S_{3 d}[\varphi]-S_{3 d}\left[\varphi^{(0)}\right]=\int \hat{\phi}\left(g_{1}\right) \mathcal{K}\left(g_{1}\right) \hat{\phi}\left(g_{2}\right) \delta\left(g_{1} g_{2}\right) \\
& -\frac{\mu}{3 !} \int[d g]^{3} \hat{\phi}\left(g_{1}\right) \hat{\phi}\left(g_{2}\right) \hat{\phi}\left(g_{3}\right) \delta\left(g_{1} g_{2} g_{3}\right)-\frac{\lambda}{4 !} \int[d g]^{4} \hat{\phi}\left(g_{1}\right) \cdots \hat{\phi}\left(g_{4}\right) \delta\left(g_{1} \cdots g_{4}\right),
\end{aligned}
$$

with the kinetic term and the 3 -valent coupling given in terms of $F$

$$
\mathcal{K}(g)=1-2\left(\int[d h] F(h)\right)^{2}-\int[d h] F(h) F(h g), \quad \text { and } \quad \frac{\mu}{3 !}=\sqrt{\frac{\lambda}{3 !}} \int[d h] F(h) .
$$


One can choose $F$ such that $\mathcal{K}(g)$ becomes the standard propagator $p^{2}-m^{2}$ with a non-zero mass [90]. We recognize then an action that is very close to the one in (4.11). From this perspective, in 3d, using a GFT to generate spinfoams we can find some degrees of freedom which can be interpreted as matter. Furthermore these matter degrees of freedom have naturally a curved momentum space. If we perform the Fourier transform (4.10), we would recover matter as propagating in a non-commutative spacetime, of the Lie algebra type.

The 4D extension of this model to recover a scalar field in $\kappa$-Minkowski space from a topological GFT (i.e. giving the BF spinfoam amplitudes) was proposed in [112]. The construction is a bit more involved than in the above example since we have to deal with non-compact groups. Furthermore momentum space in $\kappa$-Minkowski is the group $\mathrm{AN}_{3}$ which is not the one used to build the spinfoam model. One has then to use different tricks to recover this group in the GFT. For further details we refer to [112].

After these particle and field theory models we now turn to the LQG formulation of cosmological models, a promising observational window for QG phenomenology. In fact, already in the standard model of cosmology the metric is used in the quantized perturbation variables.

\section{Loop quantum cosmology}

A line of research that renders potentially observable results is Loop Quantum Cosmology (LQC). (For readers new to this subject we suggest the recent LQC reviews [40, 46, 55, 71].) In contrast to the subjects of the foregoing sections in this branch of QG phenomenology we do not consider amplifications of tiny effects in the weak gravitational field regime, but rather today's remnants of the strong gravitational regime in the early universe. Given the observational windows onto the early universe, this line of work holds promise for accessible hints of fundamental space-time structure.

We do not have solutions to full LQG that could be restricted to cosmological models. So, to model the early universe and to obtain a dynamical evolution with observable consequences, one assumes a cosmological background - usually highly symmetric, homogeneous or homogeneous and isotropic models. With a scalar inflaton field one can consider perturbations around the background by means of effective equations. From the effective equations one can derive estimates for correlation functions of quantities of scalar and tensorial type, constructed from perturbations of the connection around the isotropic case and relevant for the period of inflation. Finally these can be compared with the CMB inhomogeneities.

In homogeneous cosmological models the degrees of freedom are reduced to a finite number by symmetry reduction prior to (loop) quantization. This results in simplified operators, and particularly, in a simplified constraint algebra, tailored to the cosmological model under consideration. For such systems we often know exact or at least numerical solutions. Although not solutions of full LQG, but of a simplified offspring of LQG, these constructions are guided by the effort to be as close as possible to the full theory.

In the following we illustrate the approach to LQC with the simplest cosmological model, the Friedmann-Lemaitre-Robertson-Walker (FLRW) model with zero spatial curvature [40]. The gravitational part of this model is one-dimensional, the only geometrical dynamical variables are the scale factor $a(t)$ of the universe and the expansion velocity $\dot{a}(t)$. The Gauss and the diffeomorphism constraints do not show up explicitly, they are automatically satisfied, what remains to solve is the Hamiltonian constraint in form of a difference equation. Discreteness plays a significant role only in the very early phase of the universe, in the ensuing continuous evolution the difference equation can be approximated by a differential equation. The intermediate regime between these two phases is the domain of quantum corrections to classical equations. 
The metric of the flat FLRW model is usually given in the form

$$
g_{\mu \nu} \mathrm{d} x^{\mu} \mathrm{d} x^{\nu}=-\mathrm{d} t^{2}+a^{2}(t)\left(\mathrm{d} x_{1}^{2}+\mathrm{d} x_{2}^{2}+\mathrm{d} x_{3}^{2}\right)
$$

with a fiducial spatial Euclidian metric. As the spatial topology of the model is that of $\mathbb{R}^{3}$, one has to choose a fiducial cell $\mathcal{C}$ to obtain finite integrals in quantities like the total Hamiltonian, the symplectic structure, and others. In comoving euclidian coordinates the volume of such a cell is denoted by $V_{0}$, the corresponding geometric volume is $V=a^{3} V_{0}$. When introducing Ashtekar variables, we can, thanks to the symmetry of the model, choose the homogeneous and isotropic densitized triad and connection variables

$$
A_{a}{ }^{i}=V_{0}^{-1 / 3} c \delta_{a}{ }^{i} \quad \text { and } \quad E^{a}{ }_{i}=V_{0}^{1 / 3} p \delta^{a}{ }_{i}
$$

In terms of metric variables we have

$$
c=V_{0}^{1 / 3} \gamma \dot{a} \quad \text { and } \quad p=V_{0}^{2 / 3} a^{2},
$$

where $\gamma$ is the Barbero-Immirzi parameter. The Poisson bracket is independent of the size of the fiducial cell,

$$
\{c, p\}=\frac{8 \pi G \gamma}{3} .
$$

With these variables the gravitational phase space of homogenous and isotropic models is spanned by one canonical pair and, with a spatially constant field $\phi$ and its canonical momentum, we have finite-dimensional quantum mechanics. Approaches of this kind are summarized under the notion of Wheeler-DeWitt (WDW) theory or "Geometrodynamics", see [86].

In LQC we want to take into account discreteness and so in the spirit of LQG we construct holonomies from the connection variable. For this purpose we choose an edge of $\mathcal{C}$, whose coordinate length $V_{0}^{1 / 3}$ is multiplied by a dimensionless parameter $\mu$. Like in LQG, where SNW edges carry quanta of area, $\mu$ will later turn out to be a measure of area.

The holonomy along such an edge is

$$
h=\exp \left[\mu c \tau_{k}\right]=\cos \frac{\mu c}{2} \mathbb{I}+2 \sin \frac{\mu c}{2} \tau_{k},
$$

where $\mathbb{I}$ is the $2 \times 2$ unit matrix and $\tau_{k}$ is an $\mathfrak{s u}(2)$ element(with normalization $\left(\tau_{k}\right)^{2}=-1 / 4 \mathbb{I}$ ). Obviously it suffices to take

$$
\mathcal{N}_{\mu}(c):=e^{\frac{i \mu c}{2}}
$$

as elementary functions in the connection representation. The flux through a face of $\mathcal{C}$ is given directly by $p . \mathcal{N}$ and $p$ make up the holonomy-flux algebra of the flat FLRW model, promoted to operators, their commutator is

$$
\left[\hat{\mathcal{N}}_{\mu}(c), \hat{p}\right]=-\frac{8 \pi \gamma G \hbar}{3} \frac{\mu}{2} \hat{\mathcal{N}}_{\mu}
$$

Quantum states are represented by almost periodic functions $\Psi(c)$, i.e. countable superpositions of elementary plane wave functions

$$
\Psi(c)=\sum_{n} \alpha_{n} \exp \frac{i \mu_{n} c}{2}, \quad \alpha_{n} \in \mathbb{C}, \quad \mu_{n} \in \mathbb{R} .
$$

A brief introduction into this formalism, the Bohr compactification of the real line, may be found in [257, Chapter 28] and [55]. 
In the kinematic Hilbert space with the norm

$$
\|\Psi\|^{2}=\lim _{D \rightarrow \infty} \frac{1}{2 D} \int_{-D}^{D} \bar{\Psi}(c) \Psi(c) \mathrm{d} c=\sum_{n}\left|\alpha_{n}\right|^{2}
$$

the functions $\mathcal{N}_{\mu}$ constructed above form an orthonormal basis,

$$
\left\langle\mathcal{N}_{\mu} \mid \mathcal{N}_{\mu^{\prime}}\right\rangle=\left\langle e^{\frac{i \mu c}{2}} \mid e^{\frac{i \mu^{\prime} c}{2}}\right\rangle=\delta_{\mu, \mu^{\prime}} .
$$

Note that on the right-hand side there is the Kronecker- $\delta$. These functions are analogs of the SNW functions in LQG. The actions of the holonomy and flux operators on a state function are by multiplication and derivative, respectively,

$$
\hat{\mathcal{N}}_{\sigma} \Psi(c)=\exp \frac{i \sigma c}{2} \Psi(c), \quad \hat{p} \Psi(c)=-i \frac{8 \pi \gamma \ell_{\mathrm{P}}^{2}}{3} \frac{\mathrm{d} \Psi}{\mathrm{d} c} .
$$

It is also possible to go over to the $p$-representation, which is sometimes more convenient. Here the quantum states are functions of $\mu$ and the operators act in the following way,

$$
\hat{\mathcal{N}}_{\alpha} \Psi(\mu)=\Psi(\mu+\alpha), \quad \hat{p} \Psi(\mu)=\frac{4 \pi \gamma \ell_{\mathrm{P}}^{2}}{3} \mu \Psi(\mu),
$$

i.e. as shift operators and by multiplication. Here $\mu$, which was originally introduced as a dimensionless ratio of lengths in [40], is proportional to area, as a factor in the eigenvalue of $\hat{p}$.

The dynamics of cosmological models will be dealt with in Section 5.3. As the main goal in LQC is to be as close as possible to the full theory, an adaption of the full LQG Hamiltonian is more convenient than the simplified Hamiltonian constraint resulting from a symmetry reduced model. In this way discreteness enters the dynamics in a much more natural way.

Some more general features of LQC, applicable to cosmological models of different degrees of complexity summarize the expected LQC corrections.

- The LQG Hamiltonian constraint contains an inverse volume expression. The volume operator has a zero eigenvalue and therefore does not have a densely defined inverse, for the inverse volume an operator of its own must be constructed. This is done in such a way that for "large" volume its eigenvalues go like $V^{-1}$, but for "small" volume they do not diverge, but in the limit $V \rightarrow 0$ eventually go to zero. This construction contains one parameter, on the value of which it depends, how many Planck volumes have to be considered as "large" or "small" in the above sense, this gives rise to quantum ambiguities. The well-defined inverse volume operator is an important ingredient in resolution of the classical cosmic singularity. (See [40] for more on singularity resolution.)

- The classical Hamiltonian constraint contains the connection, which is not gauge invariant and so has no operator equivalent in the gauge-invariant Hilbert space. Like in full LQG, connection variables are replaced in one or the other way by corresponding holonomies, as in the example described above. This introduces in principle infinitely many terms of arbitrary powers of the connection, leading to corrections in the classical equations.

- There are quantum back reaction effects from fluctuations, which occur in any system, when the expectation value of the Hamiltonian operator is not the classical Hamiltonian function of the expectation values of its arguments, $\langle\hat{H}\rangle(q, p) \neq H(\langle\hat{q}\rangle,\langle\hat{p}\rangle)$. This is the case for cosmological Hamiltonians. Back reaction terms are included into an effective Hamiltonian in the effective Friedmann equations.

- Effective Poisson brackets with a correction parameter $\alpha$, constructed from correction terms, should be anomaly-free. Anomaly-freeness means that the constraints remain firstclass, which is essential for consistency and was shown explicitly in several cases, but is not established in general in LQC. 
In the following we consider holonomy corrections and inverse volume correction in dependence of a QG length parameter and their interesting interplay. Intuitively we can expect that the smaller the length scale, the smaller the holonomy corrections and the larger the inverse volume corrections, and vice versa.

\subsection{Holonomy corrections}

As above, we assume the fiducial cell $\mathcal{C}$ with comoving coordinate volume $V_{0}$ and physical volume $V=a^{3} V_{0}$ of a cosmological model partitioned into $N$ elementary building blocks of volume $v=a^{3} \frac{V_{0}}{N}$. This gives a length scale $L=v^{1 / 3}$ [58]. Setting $L=\mu V^{1 / 3}$ with $\mu$ corresponding to the state functions $|\mu\rangle$ ties $L$ to the quantum theory. Here $\mu$ appears first as a dimensionless proportionality factor of (classical) lengths; in quantum theory it is connected with fluxes, see (5.2). A typical QG density

$$
\rho_{\mathrm{QG}}=\frac{3}{8 \pi G L^{2}},
$$

becomes $\frac{3}{8 \pi}$ times the Planck density $M_{\mathrm{P}} / \ell_{\mathrm{P}}$, when $L=\ell_{\mathrm{P}}$. Polynomial terms in the connection in the LQG Hamiltonian constraint operator are replaced by holonomies (5.1) along an edge, this leads to higher-order corrections. Holonomy corrections become large when the exponent $\mu c$ is of order one.

From the classical Friedmann equation we can express the matter density in terms of $L$ and $c$,

$$
\rho=\frac{3}{8 \pi G}\left(\frac{\dot{a}}{a}\right)^{2}=\frac{3(c \mu)^{2}}{8 \pi G \gamma^{2} L^{2}} .
$$

Thus holonomy corrections are large when $\rho \approx \gamma^{-2} \rho_{\mathrm{QG}}$. As a measure for holonomy corrections we introduce

$$
\delta_{\mathrm{hol}}=\frac{\rho}{\rho_{\mathrm{QG}}}=\frac{8 \pi G}{3} L^{2} \rho .
$$

This relation implies that we may expect considerable holonomy corrections in early phases of the universe, when the density is large.

\subsection{Inverse volume corrections}

Thiemann [258] showed that expressions containing the inverse volume, like (5.3), which comes from the Hamiltonian constraint, can be classically expressed in terms of the Poisson bracket of the connection and the volume,

$$
\left\{A_{a}^{i}, V\right\}=\left\{A_{a}{ }^{i}, \int \mathrm{d}^{3} x \sqrt{|\operatorname{det} E|}\right\}=2 \pi \gamma G \epsilon^{i j k} \epsilon_{a b c} \frac{E^{b}{ }_{j} E^{c}{ }_{k}}{\sqrt{|\operatorname{det} E|}} \operatorname{sgn}(\operatorname{det} E) .
$$

So in quantum theory the Poisson bracket can be expressed by a commutator of well-defined operators, when the connection is replaced by the corresponding holonomy. This construction is at the root of the cosmological singularity problem. For holonomies with links of coordinate length $L / a$ we write

$$
\frac{1}{2} \frac{i \hbar L}{a}\left\{\widehat{A_{a}^{i}, V_{v}}\right\} \dot{e}^{a} \sim \operatorname{tr}\left(\tau^{i} \hat{h}_{v, e}\left[\hat{h}_{v, e}^{-1}, \hat{V}_{v}\right]\right)
$$

where $\dot{e}^{a}$ is the tangent vector to a link $e$ adjacent to the node $v ; h_{v, e}$ is a holonomy along a link adjacent to $v$; and $V_{v}$ is the volume of a region containing $v$. There is an ambiguity in the $\mathrm{SU}(2)$ 
representation, in which the trace is taken. The parameter labeling this ambiguity influences the scale, where the transition from the discrete quantum universe to the continuous classical universe takes place. It enables us to model the time scale of inflation [54].

In the older literature a fixed discreteness scale $\mu_{0}=$ const with respect to the fiducial metric was employed, which led to problems in the continum limit. For comparison we present the fixed lattice formulation and postpone the refined lattice to the next subsection. In this case, a volume eigenstate $|\mu\rangle$ with

$$
\hat{V}|\mu\rangle=\left(\frac{4 \pi \gamma \ell_{\mathrm{P}}^{2}}{3}|\mu|\right)^{3 / 2},
$$

and for the simplest choice of $j=1 / 2$ for the $\mathrm{SU}(2)$-representation in the trace, the inverse volume operator acts in the following way

$$
\widehat{V^{-1}}|\mu\rangle=\left|\frac{3}{4 \pi \gamma \ell_{\mathrm{P}}^{2} \mu_{0}}\left(\left\{V\left(\mu+\mu_{0}\right)\right\}^{1 / 3}-\left\{V\left(\mu-\mu_{0}\right)\right\}^{1 / 3}\right)\right|^{3} .
$$

From this, one derives the action of the self-adjoint gravitational Hamiltonian constraint operator (constructed from curvature terms of full LQG) on $|\mu\rangle$ :

$$
\begin{aligned}
\hat{H}_{\mathrm{g}}|\mu\rangle= & \frac{M_{\mathrm{P}}}{32 \sqrt{3 \pi} \gamma^{3 / 2} \mu_{0}^{3}} \\
& \times\left\{\left[R(\mu)+R\left(\mu+4 \mu_{0}\right)\right]\left|\mu+4 \mu_{0}\right\rangle+4 R(\mu)|\mu\rangle+\left[R\left(\mu-4 \mu_{0}\right)\right]\left|\mu-4 \mu_{0}\right\rangle\right\}
\end{aligned}
$$

with

$$
R(\mu)=|| \mu+\left.\mu_{0}\right|^{3 / 2}-\left|\mu-\mu_{0}\right|^{3 / 2} \mid .
$$

When the commutator in (5.4) is expressed in terms of holonomy and flux operators, its expectation values in quantum states do not have the classical relationship with the expectation values of the basic operators. Classically the flux through an elementary lattice site is $L^{2}(a)$, where $L$ is the length scale introduced in the foregoing subsection, which depends on the scale factor according to its definition. In [58] the flux operator is rewritten in the form $\hat{F}=\langle\hat{F}\rangle+$ $(\hat{F}-\langle\hat{F}\rangle)$ and the volume operator as function of $\hat{F}$ is expanded in $\hat{F}-\langle\hat{F}\rangle$. With $\langle\hat{F}\rangle=L^{2}(a)$ in lowest order one obtains a correction function $\alpha$ to classical Hamiltonians, depending on the scale factor, whose expansion for small deviations from the classical value for large $L(a)$ is

$$
\alpha(a)=1+\alpha_{0} \delta_{\mathrm{Pl}}(a)+\cdots
$$

with $\alpha_{0}$ being a constant and

$$
\delta_{\mathrm{Pl}}:=\left(\frac{\ell_{\mathrm{P}}}{L}\right)^{4} \text {. }
$$

Volume corrections become large, when $L$ is small of the order of the Planck length.

In comparison with holonomy corrections we may observe that $\delta_{\mathrm{Pl}}$ is small, when $L \gg \ell_{\mathrm{P}}$, $\rho_{\mathrm{QG}}$ becomes small and holonomy corrections become large. The relation between these two kinds of corrections can be better seen in terms of densities,

$$
\delta_{\mathrm{Pl}}=\left(\frac{8 \pi G}{3} \rho_{\mathrm{QG}} \ell_{\mathrm{P}}\right)^{2}=\left(\frac{8 \pi}{3} \frac{\rho_{\mathrm{QG}}}{\rho_{\mathrm{Pl}}}\right)^{2}=\left(\frac{8 \pi}{3} \frac{\rho}{\rho_{\mathrm{Pl}}} \delta_{\mathrm{hol}}^{-1}\right)^{2} .
$$

Inverse volume corrections depend on the ratio of the QG density to the Planck density, whereas holonomy corrections depend on the ratio between the actual density and the QG density. For 
small densities in an expanding universe, holonomy corrections decrease, but (5.6) tells us that $\delta_{\mathrm{Pl}}$ cannot simultaneously go down arbitrarily. This gives at least a lower bound for LQC corrections, from which M. Bojowald et al. in [58] derive lower bounds to correlation functions of inhomogeneities in the CMB. Here we do not have only upper bounds for LQC effects, but an estimate that gives rather narrow bounds for parameters like $\alpha_{0}$. Here we note that, as the size of the inverse volume corrections relies on the size of a fiducial cell, in [40] it is argued that they become negligible, when the limit of an infinite cell is taken, so that it extends over all $\mathbb{R}^{3}$.

The correction function $\alpha$ appears also in the effective constraint algebra, where the Poisson brackets of two smeared-out Hamiltonian constraints $H(M)$ and $H(N)$ are modified in the form

$$
\{H(M), H(N)\}=D\left[\alpha^{2} q^{a b}(N \partial M-M \partial N)\right]
$$

( $D$ is the diffeomorphism constraint). The effective algebra is, importantly, first-class, i.e. anomaly-free. Anomaly freeness was shown for not too large departures from FLRW.

In modeling inflation the inhomogeneities superposed on the FLRW background find their way into the classical Friedmann equation and the equation of motion of the scalar field in form of holonomy and inverse-volume corrections. So they enter into the basis of inflation models with different sorts of dilaton potentials and eventually emerge in the perturbation power spectrum of CMB, where it comes into contact with observations. The lower bounds of the predicted LQC corrections are only a few orders of magnitude away from the present upper observational bounds [58], so that we can hope for an experimental judgement in the not too distant future. However, see Section 5.4 for further discussion on this.

\subsection{Dynamics and lattice refinement}

In the dynamical evolution of an expanding model universe in LQC, were it rigorously derived from LQG, we might expect a steady creation of new nodes by the Hamiltonian constraint operator, which keeps the typical link length small. Without the full LQG dynamics we cannot see this creation of links. What we can do is to try to model a refinement of the SNW lattice by hand $[235,236]$.

As mentioned above, the dynamics in LQC is constructed with the aid of the LQG Hamiltonian constraint, including some kind of matter as "internal clock", usually a scalar field. Beside the inverse volume, in the Hamiltonian the curvature $F_{a b}{ }^{k}$ plays an important role. Classically it can be written as limit of holonomies around a plaquette in the $(a, b)$ plane, when the area of the plaquette goes to zero. However, due to the discreteness of the spatial geometry, in LQC this limit does not exist. The curvature term in the Hamiltonian is expressed for finite plaquettes, the area of which is chosen to be equal to the area gap $\Delta A=4 \sqrt{3} \pi \gamma \ell_{\mathrm{P}}^{2}$, the lowest nonzero eigenvalue of the LQG area operator.

The plaquettes introduce a new length scale in the classical theory, when we assume that a face of a fiducial cell $\mathcal{C}$ is partitioned into $N$ plaquettes of area $\left(\bar{\mu} V_{0}^{1 / 3}\right)^{2}$. The parameter $\bar{\mu}$ is distinct from the parameter $\mu$ which characterizes holonomies. A dynamical length scale, $\bar{\mu}$ appears in the regularization of the Hamiltonian constraint operator.

To determine a relation between $\bar{\mu}$ and the characteristic value $\mu$ of a state $|\mu\rangle=\Psi(\mu)$ which the Hamiltonian constraint operator is to act on, we take the area of a face of the fiducial cell

$$
N \Delta A=|p|=\frac{4 \pi \gamma \ell_{\mathrm{P}}^{2}}{3}|\mu|
$$

and take into account that it is covered by $N$ plaquettes,

$$
N\left(\bar{\mu} V_{0}^{1 / 3}\right)^{2}=V_{0}^{2 / 3}
$$


Eliminating $N$ we find the discreteness scale in the Hamiltonian depends on quantum states via

$$
\bar{\mu}(\mu)=\left(\frac{3 \sqrt{3}}{|\mu|}\right)^{1 / 2} .
$$

This means, when the area of a face of $\mathcal{C}$, or its physical volume $V$, grow due to a growing scale factor, the partition of a fiducial cell is refined. This is necessary for the size $\bar{\mu}$ of the quanta of geometry to remain small, when the universe expands.

The necessity for this lattice refinement is best seen in considering the classical, continuous limit. As the scale factor $a$ becomes large in the course of the dynamical evolution, the difference equation stemming from the Hamltonian constraint can be approximated by a differential equation, the WDW equation, for a smooth wave function. The wave function oscillates on scales $\sim a^{-1}$, and for growing $a$, this becomes smaller than the discreteness scale, when the latter were given by a constant and thus firmly tied to the scale factor. We refer to $[40,235,236]$ for the details.

For a fixed lattice scale $\mu_{0}$, holonomies $\exp \left(i \mu_{0} c / 2\right)$ act as simple shift operators by the constant $\mu_{0}$ on states $|\mu\rangle$, the action of $\exp (i \bar{\mu} c / 2)$ is more complicated, because $\bar{\mu}$ is a function of $\mu$. However on volume eigenstates $|\nu\rangle$ with

$$
\nu=\frac{2}{3} \mu^{3 / 2}
$$

and

$$
\hat{V}|\nu\rangle=\frac{3 \nu}{2}\left(\frac{4 \pi \gamma}{3}\right)^{3 / 2} \ell_{\mathrm{P}}^{3}|\nu\rangle
$$

the refined lattice holonomies act by a shift and the self-adjoint gravitational Hamiltonian acquires a form analogous to (5.5)

$$
\begin{aligned}
H_{\mathrm{g}}|\nu\rangle= & \frac{|\nu|}{64 \sqrt{2 \pi} \kappa \gamma^{3 / 2} \mu_{0}^{3}}\left[\frac{1}{2}\left\{U(\nu)+U\left(\nu+4 \mu_{0}\right)\right\}\left|\nu+4 \mu_{0}\right\rangle-2 U(\nu)|\nu\rangle\right. \\
& \left.+\frac{1}{2}\left\{U(\nu)+U\left(\nu-4 \mu_{0}\right)\right\}\left|\nu-4 \mu_{0}\right\rangle\right]
\end{aligned}
$$

with

$$
U(\nu)=\left|\nu+\mu_{0}\right|-\left|\nu-\mu_{0}\right|
$$

In summary, lattice refinement fulfills all the following conditions:

1) Independence of the elementary cell chosen in an open cosmological model to make integration finite.

2) Inflation becomes "natural" in the sense that an inflaton mass $M_{\mathrm{inf}} \leq 10^{2} M_{\mathrm{P}}$ is sufficient, in contrast to much lower values without lattice refinement.

3) Factor ordering in the macroscopic WDW equation becomes unique.

4) The requirement of "pre-classicality" is fulfilled, i.e. quantum corrections at large scales are avoided.

Consequences for inflation can be seen in [235]. Here we mention just a few facts about modeling inflation with and without lattice refinement. We take a wave function, depending on the volume of the universe, or $p=V^{2 / 3}$, respectively, and an inflaton field $\phi$,

$$
|\psi(p, \phi)\rangle=\sum_{\nu} \psi_{\nu}(\phi)|\nu\rangle
$$


where $|\nu\rangle$ is an eigenstate of volume, $\nu$ is related with $\mu$ by (5.7). In the continuous limit, when the evolution equation of the wave function is approximated by a differential equation, we assume

$$
\psi(p, \phi)=\Upsilon(p) \Phi(\phi)
$$

If the dynamics of $\phi$ is approximately driven by the potential $V(\phi)=V_{\phi} p^{\delta-3 / 2}$, where $V_{\phi}$ is constant and $\delta=3 / 2$ in the case of slow-roll, then this leads to an oscillating function $\Upsilon(p)$ with a separation of two successive zeros

$$
\Delta p=\frac{\pi}{\sqrt{\beta V_{\phi}}} p^{(1-2 \delta) / 4}, \quad \text { with } \quad \beta=\frac{12}{\pi G \hbar^{2}} .
$$

In the continuum limit the wave function must vary slowly on the discreteness scale $\bar{\mu}$ of $\mathrm{QG}$ (pre-classicality, see [54]). This can be formulated in the way that the distance between two zeros in terms of $\mu, \Delta \mu=\frac{3}{4 \pi \gamma \ell_{\mathrm{P}}^{2}} \Delta p$ must be at least equal to $4 \bar{\mu}$, which yields the condition of pre-classicality

$$
\Delta p>16(\pi \gamma)^{3 / 2} \ell_{\mathrm{P}}^{3} p^{-1 / 2},
$$

which leads to an upper bound of the inflaton potential, $V(\phi) \leq 2.35 \cdot 10^{-2} \ell_{\mathrm{P}}^{-4}$, in contrast to $V(\phi) \ll 10^{-28} \ell_{\mathrm{P}}^{-4}$ for fixed lattice. To be in accordance with the COBE-DMR measurements, the potential must further satisfy

$$
\frac{[V(\phi)]^{3 / 2}}{V^{\prime}(\phi)} \approx 5.2 \cdot 10^{-4} M_{\mathrm{P}}^{3}
$$

If we choose an inflaton potential $V(\phi) \approx \frac{m^{2} \phi^{2}}{2}$ then we obtain, from the least two conditions, $m \leq 10 M_{\mathrm{P}}$, compared with $m \leq 70\left(e^{-2 N_{\mathrm{cl}}}\right) M_{\mathrm{P}}$ for the fixed lattice. $N_{\mathrm{cl}}$ is the number of $e-$ folds. The condition that a significant proportion of the inflationary regime takes place during the classical era imposes a condition on the inflaton mass, which is much more natural for the refined lattice.

\subsection{Loop Quantum Cosmology: possible observational consequences}

With the development of LQC and recent observational missions in cosmology there are rich veins of work to explore in the phenomenology of the early universe. There are many approaches to this work. As yet there is no consensus on the best route from the discrete quantum geometry of LQG to observable cosmological predictions. In this review of LQC phenomenology, which is very brief, we do not attempt a comprehensive review of the literature but rather provide a guide to starting points for further exploration of these veins of work. Our scope is further reduced by focusing on observational signatures that will be accessible in the near future.

The current best observational window on the early universe is the power spectrum of small angular fluctuations in the cosmic microwave background (CMB) radiation. In the standard inflationary model these fluctuations are generated by scalar and tensor perturbations. Thus, the power spectra of the scalar and tensor perturbations are the key tools for investigations of cosmic background radiation in electromagnetic and gravitational sectors. Because of the difference in scales at which decoupling occurs, the gravitational wave background originates at an earlier epoch than the CMB, thus allowing a view into the very early universe. However, observation of the gravitational background remains a huge experimental challenge.

The now-familiar plot of the CMB power spectrum is of angular correlations of the temperature-temperature, or "TT", power spectrum. Polarization modes of the CMB are decomposed 
into curl-free electric $E$-modes and gradient-free magnetic $B$-modes. The $B$-mode power spectrum arises from two effects: directly from primordial gravitational waves (the tensor modes) and indirectly through lensing from the conversion of $E$-mode to $B$-mode. Therefore, most intriguingly, it may be possible to study tensor modes from polarization measurements of the CMB, without directly observing the gravitational wave background.

As in the case of LV, there are a number of different perturbation frameworks under development. For instance in one class of widely-used frameworks, the background space-time enjoys LQC modifications (corrections due to holonomy, inverse volume, or both) and the perturbations take a form similar to the perturbations in FLRW cosmologies, where the linear perturbations of Einstein's equations are quantized. Because the background does not follow Einstein's equations in this framework it is not immediately clear that perturbation equations are consistent. There may exist a consistent set, but this issue is currently not resolved.

In another framework (see, e.g. [40, Section VI.C]), the classical theory is first reduced by decomposing the gravitational phase space into homogeneous and purely inhomogeneous parts (for matter as well as gravitational variables); the linear perturbations are entirely within the inhomogeneous phase space. Then both the background and the linear perturbations are quantized with LQC techniques. In related work of [34,84], a quantum scalar field is analyzed on a quantized Bianchi I background. This work provides a framework for perturbations on an effective 'dressed' quantum geometry that may also contain back reaction.

Most current studies are some blend of the traditional framework of cosmological perturbations and an effective LQC framework. The formulation of these frameworks are currently a matter of lively debate; see, for instance, [40, Section VI.D], [58, Sections 2.3.4], and [261]. Nevertheless there is an impressive body of work in developing the phenomenology of the very early universe.

Loopy modifications to the power spectrum have been derived. LQC offers at least two modifications to the usual scenario, holonomy and inverse-volume or inverse-triad corrections as discussed in the last section. Both these corrections have been incorporated in models of the background space-time using LQC methods. As yet we lack a comprehensive study of all the LQC effects on the scalar and tensor perturbations. Nonetheless there are many studies analyzing how specific models of LQC corrections affect the power spectra. We will mention two lines of work, one on affects in the power spectrum of scalar and tensor perturbations and the other on the chirality of tensor perturbations. This last work is outside the symmetry reduced LQC models and is included here as it concerns tensor perturbations.

\subsubsection{Scalar and tensor perturbations}

In the effective Friedmann equation framework scalar perturbations with inverse triad corrections are discussed in [57,60-62] and with holonomy corrections in [70,261,262]. In [70] correction terms were introduced without a gauge choice with the result that the perturbation equations are anomaly-free. Tensor modes in the same framework are discussed in $[57,59,82,117,204]$ with inverse triad corrections and in [59, 115, 115, 200-202] with holonomy corrections.

Starting with [59] the work in [116, 204] develops a phenomenological model of the tensor modes within a model bouncing cosmology with a single massive scalar field. They find that the effects can be modeled with two parameters [116], one "bump parameter" is simply related to the inflaton mass. The second parameter, a transition wave number, is related in a complicated way to the critical density and the scalar potential energy-critical density ratio at the bounce. The authors find that the tensor power spectrum is suppressed in the infra-red regime, agrees with the standard general relativistic picture in the UV, and has both an increase in amplitude and damped oscillations at intermediate scales. This work suggests that the next generation 
$B$-mode experiments could provide a successful constraints on the model parameters [116]. For more on this approach see [115-117,201,203,204].

The power spectra of scalar and tensor modes, with inverse triad corrections, are derived in the inflationary scenario with effective Friedmann equations in $[57,58,72]$. In $[57,58]$ the corrections are parameterized with corrections related to the area gap, a parameterization of quantization ambiguities, and how the number of lattice sites changes in the evolution of the cosmology. This leads to an enhancement of power on large angular scales. The model is compared to WMAP 7 year data as well as other astronomical surveys in [58].

Normally in the inflationary scenario, because of the rapid expansion, the universe is in its vacuum state shortly after the onset of inflation. It was pointed out in $[7,8]$, however, that if pre-inflationary physics in LQC led to a non-vacuum state then spontaneous generation of quanta would have observational consequences in terms of non-Gaussianities in the CMB and in the distribution of galaxies.

The wealth of phenomenological models means that there is guidance as to many effects arising from aspects of LQG. The field has evolved to the point where these models can be directly compared with current data. But there remain many questions on the derivation of these effects from a more fundamental level, both from LQC and from LQG. For instance, the parameter capturing the phenomenology of inverse volume corrections depends on the fiducial volume [57]. While the parameter can be fixed by the size of the Hubble horizon at horizon crossing the parameterization is debated. So the status of inverse volume corrections in the presence of inhomogeneities is a matter of current debate (particularly when the spatial topology is non-compact), see e.g. [58, Section 2.4] and [40, Section VI.D].

\subsubsection{Chirality of tensor perturbations}

Working with the Ashtekar-Barbero connection formalism and deriving the tensor perturbations in a de Sitter background, Magueijo and collaborators find that the graviton modes have a chiral asymmetry in the vacuum energy and fluctuations if the Immirzi parameter has an imaginary part $[48,49,177,178]$. This is significant as the chirality would leave an imprint on the polarization of the cosmic microwave background and might be observed with the PLANCK mission. The asymmetry depends on operator ordering.

\subsection{Phenomenology of black hole evaporation}

The subject of this subsection is closely related to cosmology and has potentially observable consequences. In [206] semiclassical models of Schwarzschild and Reissner-Nordström black holes are presented. They are based on LQG's discreteness of area and a resulting repulsive force at extremal densities. With these ingredients the space-time metric outside "heavy" black holes (with respect to the Planck mass) is only slightly modified in relation to the classical form, but inside the horizon the singularity is smoothed out and in the limit when the radial coordinate goes to zero the metric becomes asymptotically Minkowski. By the introduction of the new coordinate $R=a_{0} / r$, where $a_{0}$ is the LQG-inspired minimal area, the regularized metric is shown to be self-dual in the sense of T-duality: An observer at $R \rightarrow \infty$ sees a black hole with mass $\sim m_{\mathrm{Pl}} / m$, when $m$ is the mass of the black hole seen by observers in the asymptotic flat region $r \rightarrow \infty$. For "light" (= sub-Planckian) black holes also the outside metric is modified considerably.

"Light" black holes do not evaporate completely, although they would emit high-energy radiation at an extremely low rate. They are supposed to explain two cosmological puzzles: Being practically stable, ultralight black holes created during the inflation process could account for dark matter as well as they could be the so far unknown source for UHECR. 
In the sequel $[47,136]$ it was shown that the discreteness of area leads to features that distinguish black hole evaporation spectra based on LQG. They are very distinctly discrete in contrast to the classical Hawking spectrum and observation, should it become possible, should be able to distinguish LQG from other underlying QG theories.

\section{Conclusions}

In this review we have described ways in which LQG, mainly by means of discreteness of the spatial geometry, may lead to experimentally viable predictions. We discussed ways in which the discreteness may (or may not) lead to a large variety of modifications of special relativity, particle physics and field theory in the weak field limit. In Sections 3 and 4 effective particle and field theory frameworks are presented in some detail. Where possible we have given current observational bounds on the models. In these sections, as well as in the LQC section, we have pointed out numerous approaches and some of the theoretical and experimental open problems. Many of these are collected below.

Of course QG and QG phenomenology remain open problems. We lack strong ties between observationally accessible models and LQG. These models have ansätze that are often in striking contradiction with each other and none has a clear support from observations. Furthermore, should any of the effective models presented here be favored by experimental data in the near future, this will hardly point uniquely at one of the fundamental theories, or to a certain version of them ${ }^{11}$. On the other hand, this field has seen tremendous progress since the mid-90's when it was tacitly assumed that there were essentially no experimentally accessible windows into QG. Quite the contrary, now there are many avenues to explore QG effects and stringent bounds have already been placed on effects originating at the Planck scale. These developments are an essential first step toward a physically viable quantum gravity theory.

Concluding, the subject of LQG phenomenology, and of QG phenomenology in general, is now far reaching. We expect that QG phenomenology will remain a very active field and will hopefully bring new perspectives and clarity on the ad hoc assumptions and models. Indeed, in spite of its shortcomings, phenomenology is indispensable for LQG, or any other quantum theory of gravity, if it is to become a physical theory.

\section{A Elements of LQG}

In the first part of this appendix there is an overview of the basics of LQG. In the second part we very briefly review the theory's kinematics. For more details the reader should consult the recent brief reviews by Rovelli [227] and Sahlmann [232]. For longer reviews the reader should consult $[35,212,255,256]$ and the texts $[226,257]$.

LQG is a quantization of GR. Due to the special features of GR it looks in many points quite different from other quantum field theories:

- LQG takes into account that space and time are not an external background for physics, but part of physical dynamics.

- Gravity is self-interacting, but the self-interaction cannot be treated perturbatively, because the theory is non-renormalizable.

- Due to general covariance of GR the gauge group is the group of diffeomorphisms, not the Poincaré group.

\footnotetext{
11 "In this situation a possible conclusion is that today is too early to search for a fundamental QG theory and that one should in the meantime rather look for an "old quantum theory of gravity" in the style of Bohr and Sommerfeld", quoting G. Amelino-Camelia at the workshop "Experimental Search for QG 2010", Stockholm. Such an approach was recently successfully employed in the investigation of the volume operator [52].
} 
Whereas non-linearity is shared with other QFTs, the issue of dynamical space-time is unique for gravity.

For comparison recall that usual QFTs deal with fields on either Minkowski space or some curved Riemannian space with a given metric and the corresponding Levi-Civita connection. LQG, on the other hand, is a QFT on a manifold, a priori without further structure, and the geometric properties of physical space are realized in form of dynamical fields. Concretely, in LQG the basic field variables are not metric components, as in GR, but orthogonal bases (triads) in the tangent space of every point in three-dimensional space and the connection.

The introduction of triads brings further gauge degrees of freedom into the game, namely local rotations, i.e. elements of the group $\mathrm{SO}(3)$ (or SU(2)). Further, due to space-time covariance of GR, the embedding of the spatial 3-manifold into a 4-manifold and the choice of a time coordinate is also a matter of gauge. In the canonical formalism this is reflected by the appearance of a further gauge generator.

As common in gauge theories, gauge generators are constraints. In the present case the Gauss constraint, which has a formal analogy to the Gauss law in electrostatics, generates triad rotations. The theory also has the diffeomorphism constraint and the Hamiltonian constraint, which generates transitions from one spacelike 3-manifold to another one. In LQG these constraints are imposed as operators, which annihilate physical, i.e. gauge-invariant, quantum states. This leads immediately to a surprising feature in canonical QG: The propagation from a hypersurface to the next one being a gauge transformation, all physical states are invariant under these transitions and there is no physical time evolution. Gauge-invariant states contain all the history of a state of the gravitational field. This was pointed out long before the advent of LQG [86]. Time evolution must be introduced in an operational way in relation to some suitable kind of matter, which is coupled to gravity and may be considered as clock.

The problem of non-linearity, coming from gravity's self interaction, is more a technical than a conceptual problem. Non-linearity in interacting QFTs in the standard model is successfully dealt with by renormalization methods. The non-renormalizability of GR appears as a serious obstacle, but it can be traced back to the background - splitting the metric into a background, e.g. Minkowski space, and a (small) field on it in the form $g_{i k}=\eta_{i k}+\psi_{i k},\left|\psi_{i k}\right| \ll 1$ and quantizing "the ripples $\psi_{i k}$ on the background" does not work. The conclusion, which is drawn from this in LQG, is that only spatial geometry as a whole has a chance to be successfully quantized.

Presently the success and limitations of LQG can be summarized very briefly in the following way. Local connection components are not gauge invariant, they are not even tensor components. Constructing gauge-invariant quantities from them is possible by means of closed contour integrals, so-called Wilson loops. Their further development are holonomies and spin networks. They introduce non-locality into the theory. In consequence, metric quantities (which are not introduced from the beginning as basic variables), like area and volume, turn out to have discrete spectra with a fundamental role of the Planck length. In other words, LQG yields "quanta of space" or "atoms of geometry". The existence of a minimal length provides a natural ultraviolet cutoff for other QFTs. The main open problem is the non-linearity of the Hamiltonian constraint. Thiemann [258] succeeded to formulate it as well-defined operator in several versions, but there remain some ambiguities. A more recent approach is the master constraint programme [257]. The problem of the Hamiltonian constraint and time evolution have been satisfactorily solved in simplified models, mainly in cosmology.

Concerning technicalities, at the very basis of LQG stands a $(3+1)$ decomposition of spacetime and a canonical formulation of GR in terms of densitized triad variables $E^{a}{ }_{i}$ and connection variables $A_{a}{ }^{i}:=\Gamma_{a}^{i}-\gamma K_{a}^{i}$, which are canonically conjugate, on the spatial 3 -manifold ${ }^{12}$. The

\footnotetext{
${ }^{12}$ The index $a$ is spatial index and $i$ is an $\mathfrak{s u}(2)$ Lie algebra index.
} 
connection depends on both the spin connection $\Gamma_{a}^{i}$ and the extrinsic curvature $K_{a}^{i}$. The parameter $\gamma$ is the Barbero-Immirzi parameter, which may be fixed by matching with the BekensteinHawking black hole entropy formula, see e.g. [197].

In the connection representation, the quantum Hilbert space is spanned by functionals of the connection. A convenient basis, invariant under $\mathrm{SU}(2)$ gauge transformations of the triads, is provided by spin network (SNW) functions, defined with the aid of graphs $\Gamma$, where to each edge or link $e_{I}$ a representation of $\mathrm{SU}(2)$, corresponding to a spin $j$, is associated, the "color" of the edge. SNW functions are constructed from the path ordered exponential of the connection, the holonomies

$$
h_{e_{I}}(A):=\mathcal{P} \exp \left(-\int_{e_{I}} \dot{e}^{a} A_{a}^{i} \tau_{i}^{(j)}\right),
$$

where $\dot{e}^{a}$ is the tangent vector of the edge $e_{I}$ and $\tau_{i}^{(j)}$ the generator of the representation of $\mathrm{SU}(2)$ with spin $j$. A SNW function based on a graph $\Gamma$ with $N$ edges has the following form

$$
\Psi_{\Gamma}(A)=\psi\left(h_{e_{1}}(A), \ldots, h_{e_{N}}(A)\right) .
$$

The holonomies are connected by intertwiners at the nodes or vertices in such a way that $\psi$ is a scalar function. The functions $\Psi_{\Gamma}(A)$, having a finite number of arguments, namely the number of edges of $\Gamma$, are called cylindrical functions. They may be considered as coordinates on the space of smooth connections modulo gauge transformations, denoted by $\mathcal{A} / \mathcal{G}$. The Hilbert space of LQG is the closure of the space of cylindrical functions on generalized (distributional) connections modulo gauge transformations with the Ashtekar-Isham-Lewandowski measure [33, 36,37], constructed from the Haar measure on $\mathrm{SU}(2)$.

On this Hilbert space the configuration variable $A_{a}{ }^{i}$ would act as a multiplication operator, were it well-defined, and the momentum variable $E^{a}{ }_{i}$ as a functional derivative with respect to $A_{a}{ }^{i}$. As is common in quantum field theory, elementary variables do not enter quantum theory as operators, but as operator-valued distributions, which have to be regularized by integrating out with some test functions. In the case of the connection, the above defined holonomy operators (A.1) arise from integrating $A_{a}{ }^{i}$ in one dimension, which is natural for one-forms. These operators either add a holonomy along an link present in a SNW, or create a new link.

The momentum variable $E^{a}{ }_{i}$ is a vector density, which can be associated with a two-form $\eta_{a b c} E^{a}{ }_{i}$ with the aid of the Levi-Civita density $\eta_{a b c}$. So it is natural to smear it out by integration over a two-dimensional surface. Let a surface $\mathcal{S}$ be defined by $\left(\sigma^{1}, \sigma^{2}\right) \rightarrow x^{a}\left(\sigma^{1}, \sigma^{2}\right)$, where $\vec{\sigma}=\left(\sigma^{1}, \sigma^{2}\right)$ are coordinates of $\mathcal{S}$ and $x^{a}$ coordinates in the three-dimensional space, where $\mathcal{S}$ is embedded. Then

$$
E_{i}(\mathcal{S}):=-i \hbar \int_{\mathcal{S}} \mathrm{d}^{2} \sigma n_{a}(\vec{\sigma}) \frac{\delta}{\delta A_{a}{ }^{i}(x(\vec{\sigma}))}
$$

is a well-defined operator, the flux operator of the field $E^{a}{ }_{i}$ through $\mathcal{S}$ with

$$
n_{a}(\vec{\sigma})=\epsilon_{a b c} \frac{\partial x^{b}(\vec{\sigma})}{\partial \sigma^{1}} \frac{\partial x^{c}(\vec{\sigma})}{\partial \sigma^{2}}
$$

being the one-form normal to $\mathcal{S}$. The action on a holonomy of a link $e$ crossing $\mathcal{S}$ is

$$
E_{i}(\mathcal{S}) h_{e}(A)= \pm i \hbar h_{e_{1}}(A) \tau_{i}^{(j)} h_{e_{2}}(A)
$$

where $e_{1}$ and $e_{2}$ are two parts of the link $e$, divided by the intersection point with $\mathcal{S}$. The sign depends on the relative orientation of $e$ and $\mathcal{S} . E_{i}(\mathcal{S})$ inserts a generator at the intersection point; if $e$ and $\mathcal{S}$ do not intersect, the action is zero. The algebra of these basic operators is called the holonomy-flux algebra. 
Minkowski space is represented in LQG by a state of the gravitational field, which is not the vacuum state, but a superposition of excited SNW states. Discreteness comes in a natural way from the "polymeric" structure of the SNWs, suggesting the presence of QG effects even in flat space.

The above formulation is in the "embedded framework" of LQG. This has the advantage of having clear ties to the classical theory but in the kinematic Hilbert space is non-seperable. In addition the state space has physically mysterious continuous moduli that label equivalence classes of diffeomorphism invariant states [123]. Partly in response to these difficulties an alternate framework has received increasing attention. The combinatorial framework of LQG ${ }^{13}$ was introduced by Zapata [266,267] and recently used as the kinematic setting for spin foam models in the review [223]. In this framework the kinematical Hilbert space is separable and is free of the mysterious moduli.

\section{Acknowledgements}

Thanks to Stefano Liberati, Mathew Mewes, and Jorge Pullin for correspondence and to Stefano Liberati and Luca Maccione for permission to use their figures. We also thank the anonymous reviewers for their helpful suggestions. F.H. was supported by the Ministry of Education of the Czech Republic, contract no. MSM 0021622409.

\section{References}

[1] Abbasi R.U. et al. (High Resolution Fly's Eye Collaboration), First observation of the Greisen-ZatsepinKuzmin suppression, Phys. Rev. Lett. 100 (2008), 101101, 5 pages, astro-ph/0703099.

[2] Abdo A.A. et al. (Fermi GBM/LAT Collaborations), A limit on the variation of the speed of light arising from quantum gravity effects, Nature 462 (2009), 331-334.

[3] Abdo A.A. et al. (Fermi GBM/LAT Collaborations), Fermi observations of high-energy gamma-ray emission from GRB 080916C, Science 323 (2009), 1688-1693, arXiv:0908.1832.

[4] Abraham J. et al. (Auger Collaboration), Correlation of the highest-energy cosmic rays with nearby extragalactic objects, Science 318 (2007), 938-943, arXiv:0711.2256.

[5] Abraham J. et al. (Auger Collaboration), Upper limit on the cosmic-ray photon flux above $10^{19} \mathrm{eV}$ using the surface detector of the Pierre Auger observatory, Astropart. Phys. 29 (2008), 243-256, arXiv:0712.1147.

[6] Agostini A., Amelino-Camelia G., Arzano M., Marcianò A., Tacchi R.A., Generalizing the Noether theorem for Hopf-algebra spacetime symmetries, Modern Phys. Lett. A 22 (2007), 1779-1786, hep-th/0607221.

[7] Agullo I., Observational signatures in LQC?, Talk at International Loop Quantum Gravity Seminar (March 29, 2011), available at http://relativity.phys.lsu.edu/ilqgs/agullo032911.pdf.

[8] Agullo I., Parker L., Non-Gaussianities and the stimulated creation of quanta in the inflationary universe, Phys. Rev. D 83 (2011), 063526, 16 pages, arXiv:1010.5766.

[9] Aharonian F. et al. (HEGRA Collaboration), The Crab Nebula and Pulsar between $500 \mathrm{GeV}$ and $80 \mathrm{TeV}$ : observations with the HEGRA stereoscopic air Cherenkov telescopes, Astrophys. J. 614 (2004), 897-913, astro-ph/0407118.

[10] Alfaro J., Morales-Técotl H.A., Reyes M., Urrutia L.F., Alternative approaches to Lorentz violation invariance in loop quantum gravity inspired models, Phys. Rev. D 70 (2004), 084002, 5 pages, gr-qc/0404113.

[11] Alfaro J., Morales-Técotl H.A., Urrutia L.F., Quantum gravity corrections to neutrino propagation, Phys. Rev. Lett. 84 (2000), 2318-2321, gr-qc/9909079.

[12] Alfaro J., Morales-Técotl H.A., Urrutia L.F., Loop quantum gravity and light propagation, Phys. Rev. D 65 (2002), 103509, 18 pages, hep-th/0108061.

[13] Alfaro J., Morales-Técotl H.A., Urrutia L.F., Quantum gravity and spin-1/2 particle effective dynamics, Phys. Rev. D 66 (2002), 124006, 19 pages, hep-th/0208192.

\footnotetext{
${ }^{13}$ In the "combinatorial framework", the combinatorics refers to an approach to the topology of space while in the "combinatorics of the node" the combinatorics refers to the combinatorics of SU(2) representations.
} 
[14] Aloisio R., Galante A., Grillo A., Liberati S., Luzio E., Méndez F., Deformed special relativity as an effective theory of measurements on quantum gravitational backgrounds, Phys. Rev. D 73 (2006), 045020, 11 pages, gr-qc/0511031.

[15] Aloisio R., Galante A., Grillo A.F., Liberati S., Luzio E., Méndez F., Modified special relativity on a fluctuating spacetime, Phys. Rev. D $\mathbf{7 4}$ (2006), 085017, 7 pages, gr-qc/0607024.

[16] Amelino-Camelia G., Doubly-special relativity: facts, myths and some key open issues, in Recent Developments in Theoretical Physics, Stat. Sci. Interdiscip. Res., Vol. 9, World Sci. Publ., Hackensack, NJ, 2010, 123-170, arXiv:1003.3942.

[17] Amelino-Camelia G., Particle-dependent deformations of Lorentz symmetry, arXiv:1111.5643.

[18] Amelino-Camelia G., Quantum gravity phenomenology, arXiv:0806.0339.

[19] Amelino-Camelia G., Relativity in space-times with short-distance structure governed by an observerindependent (Planckian) length scale, Internat. J. Modern Phys. D 11 (2002), 35-60, gr-qc/0012051.

[20] Amelino-Camelia G., Briscese F., Gubitosi G., Marcianò A., Martinetti P., Mercati F., Noether analysis of the twisted Hopf symmetries of canonical noncommutative spacetimes, Phys. Rev. D 78 (2008), 025005, 8 pages, arXiv:0709.4600.

[21] Amelino-Camelia G., Ellis J., Mavromatos N.E., Nanopoulos D.V., Sarkar S., Potential sensitivity of gammaray burster observations to wave dispersion in vacuo, Nature 293 (1998), 763-765, astro-ph/9712103.

[22] Amelino-Camelia G., Freidel L., Kowalski-Glikman J., Smolin L., Relative locality: a deepening of the relativity principle, Gen. Relativity Gravitation 43 (2011), 2547-2553, arXiv:1106.0313.

[23] Amelino-Camelia G., Freidel L., Kowalski-Glikman J., Smolin L., The principle of relative locality, Phys. Rev. D 84 (2011), 084010, 13 pages, arXiv:1101.0931.

[24] Amelino-Camelia G., Laemmerzahl C., Mercati F., Tino G.M., Constraining the energy-momentum dispersion relation with Planck-scale sensitivity using cold atoms, Phys. Rev. Lett. 103 (2009), 171302, 4 pages, arXiv:0911.1020.

[25] Amelino-Camelia G., Loret N., Mandanici G., Mercati F., UV and IR quantum-spacetime effects for the Chandrasekhar model, arXiv:0906.2016.

[26] Amelino-Camelia G., Majid S., Waves on noncommutative space-time and gamma-ray bursts, Internat. J. Modern Phys. A 15 (2000), 4301-4323, hep-th/9907110.

[27] Amelino-Camelia G., Malassa M., Mercati F., Rosati G., Taming nonlocality in theories with Planck-scale deformed Lorentz symmetry, Phys. Rev. Lett. 106 (2011), 071301, 4 pages, arXiv:1006.2126.

[28] Amelino-Camelia G., Mandanici G., Procaccini A., Kowalski-Glikman J., Phenomenology of doubly special relativity, Internat. J. Modern Phys. A 20 (2005), 6007-6037, gr-qc/0312124.

[29] Antonov E.E., Dedenko L.G., Kirillov A.A., Roganova T.M., Fedorova G.F., Fedunin E.Y., Test of Lorentz invariance through observation of the longitudinal development of ultrahigh-energy extensive air showers, JETP Lett. 73 (2001), 446-450.

[30] Arzano M., Benedetti D., Rainbow statistics, Internat. J. Modern Phys. A 24 (2009), 4623-4641, arXiv:0809.0889.

[31] Arzano M., Kowalski-Glikman J., Walkus A., A bound on Planck-scale modifications of the energymomentum composition rule from atomic interferometry, Europhys. Lett. 90 (2010), 50004, 4 pages, arXiv:0912.2712.

[32] Ashtekar A., Corichi A., Zapata J.A., Quantum theory of geometry. III. Non-commutativity of Riemannian structures, Classical Quantum Gravity 15 (1998), 2955-2972, gr-qc/9806041.

[33] Ashtekar A., Isham C.J., Representations of the holonomy algebras of gravity and nonabelian gauge theories, Classical Quantum Gravity 9 (1992), 1433-1467, hep-th/9202053.

[34] Ashtekar A., Kaminski W., Lewandowski J., Quantum field theory on a cosmological, quantum space-time, Phys. Rev. D 79 (2009), 064030, 12 pages, arXiv:0901.0933.

[35] Ashtekar A., Lewandowski J., Background independent quantum gravity: a status report, Classical Quantum Gravity 21 (2004), R53-R152, gr-qc/0404018.

[36] Ashtekar A., Lewandowski J., Differential geometry on the space of connections via graphs and projective limits, J. Geom. Phys. 17 (1995), 191-230, hep-th/9412073.

[37] Ashtekar A., Lewandowski J., Projective techniques and functional integration for gauge theories, J. Math. Phys. 36 (1995), 2170-2191, gr-qc/9411046. 
[38] Ashtekar A., Lewandowski J., Quantum theory of geometry. I. Area operators, Classical Quantum Gravity 14 (1997), A55-A81, gr-qc/9602046.

[39] Ashtekar A., Lewandowski J., Quantum theory of geometry. II. Volume operators, Adv. Theor. Math. Phys. 1 (1997), 388-429, gr-qc/9711031.

[40] Ashtekar A., Singh P., Loop quantum cosmology: a status report, Classical Quantum Gravity 28 (2011), 213001, 122 pages, arXiv:1108.0893.

[41] Bahns D., Doplicher S., Fredenhagen K., Piacitelli G., Ultraviolet finite quantum field theory on quantum spacetime, Comm. Math. Phys. 237 (2003), 221-241, hep-th/0301100.

[42] Balachandran A.P., Ibort A., Marmo G., Martone M., Inequivalence of quantum field theories on noncommutative spacetimes: Moyal versus Wick-Voros planes, Phys. Rev. D 81 (2010), 085017, 8 pages, arXiv:0910.4779.

[43] Balachandran A.P., Ibort A., Marmo G., Martone M., Quantum fields on noncommutative spacetimes: theory and phenomenology, SIGMA 6 (2010), 052, 22 pages, arXiv:1003.4356.

[44] Balachandran A.P., Mangano G., Pinzul A., Vaidya S., Spin and statistics on the Groenewold-Moyal plane: Pauli-forbidden levels and transitions, Internat. J. Modern Phys. A 21 (2006), 3111-3126, hep-th/0508002.

[45] Balachandran A.P., Pinzul A., Qureshi B.A., UV-IR mixing in non-commutative plane, Phys. Lett. B 634 (2006), 434-436, hep-th/0508151.

[46] Banerjee K., Calcagni G., Martín-Benito M., Introduction to loop quantum cosmology, SIGMA 8 (2012), 016, 73 pages, arXiv:1109.6801.

[47] Barrau A., Cailleteau T., Cao X., Diaz-Polo J., Grain J., Probing loop quantum gravity with evaporating black holes, Phys. Rev. Lett. 107 (2011), 251301, 5 pages, arXiv:1109.4239.

[48] Bethke L., Magueijo J., Chirality of tensor perturbations for complex values of the Immirzi parameter, arXiv:1108.0816.

[49] Bethke L., Magueijo J., Inflationary tensor fluctuations, as viewed by Ashtekar variables and their generalizations, Phys. Rev. D 84 (2011), 024014, 17 pages, arXiv:1104.1800.

[50] Bianchi E., The length operator in loop quantum gravity, Nuclear Phys. B 807 (2009), 591-624, arXiv:0806.4710.

[51] Bianchi E., Doná P., Speziale S., Polyhedra in loop quantum gravity, Phys. Rev. D 83 (2011), 044035 , 17 pages, arXiv:1009.3402.

[52] Bianchi E., Haggard H., Discreteness of the volume of space from Bohr-Sommerfeld quantization, Phys. Rev. Lett. 107 (2011), 011301, 4 pages, arXiv:1102.5439.

[53] Bluhm R., Lorentz and CPT tests in matter and antimatter, Nuclear Instrum. Meth. Phys. Res. B 221 (2204), 6-11, hep-ph/0308281.

[54] Bojowald M., Dynamical initial conditions in quantum cosmology, Phys. Rev. Lett. 87 (2001), 121301, 4 pages, gr-qc/0104072.

[55] Bojowald M., Quantum cosmology: a fundamental description of the universe, Lecture Notes in Physics, Vol. 835, Springer, New York, 2011.

[56] Bojowald M., Quantum Riemannian geometry and black holes, in Trends in Quantum Gravity Research, Editor D.C. Moore, Nova Science Publishers, Inc., New York, 2006, 219-260, arXiv:0905.4916.

[57] Bojowald M., Calcagni G., Inflationary observables in loop quantum cosmology, J. Cosmol. Astropart. Phys. 2011 (2011), no. 3, 032, 35 pages, arXiv:1011.2779.

[58] Bojowald M., Calcagni G., Tsujikawa S., Observational test of inflation in loop quantum cosmology, J. Cosmol. Astropart. Phys. 2011 (2011), no. 11, 046, 32 pages, arXiv:1107.1540.

[59] Bojowald M., Hossain G.M., Loop quantum gravity corrections to gravitational wave dispersion, Phys. Rev. D 77 (2008), 023508, 14 pages, arXiv:0709.2365.

[60] Bojowald M., Hossain G.M., Kagan M., Shankaranarayanan S., Anomaly freedom in perturbative loop quantum gravity, Phys. Rev. D $\mathbf{7 8}$ (2008), 063547, 31 pages, arXiv:0806.3929.

[61] Bojowald M., Hossain G.M., Kagan M., Shankaranarayanan S., Gauge invariant cosmological perturbation equations with corrections from loop quantum gravity, Phys. Rev. D 79 (2009), 043505, 21 pages, arXiv:0811.1572.

[62] Bojowald M., Kagan M., Singh P., Hernández H.H., Skirzewski A., Hamiltonian cosmological perturbation theory with loop quantum gravity corrections, Phys. Rev. D 74 (2006), 123512, 13 pages, gr-qc/0609057. 
[63] Bolokhov P.A., Nibbelink S.G., Pospelov M., Lorentz violating supersymmetric quantum electrodynamics, Phys. Rev. D $\mathbf{7 2}$ (2005), 103003, 17 pages, hep-ph/0505029.

[64] Borris M., Verch R., Dirac field on Moyal-Minkowski spacetime and non-commutative potential scattering, Comm. Math. Phys. 293 (2010), 399-448, arXiv:0812.0786.

[65] Boulatov D.V., A model of three-dimensional lattice gravity, Modern Phys. Lett. A 7 (1992), 1629-1646, hep-th/9202074.

[66] Brunnemann J., Rideout D., Properties of the volume operator in loop quantum gravity. I. Results, Classical Quantum Gravity 25 (2008), 065001, 32 pages, arXiv:0706.0469.

[67] Brunnemann J., Rideout D., Properties of the volume operator in loop quantum gravity. II. Detailed presentation, Classical Quantum Gravity 25 (2008), 065002, 104 pages, arXiv:0706.0382.

[68] Brunnemann J., Thiemann T., Simplification of the spectral analysis of the volume operator in loop quantum gravity, Classical Quantum Gravity 23 (2006), 1289-1346, gr-qc/0405060.

[69] Bruno N.R., Amelino-Camelia G., Kowalski-Glikman J., Deformed boost transformations that saturate at the Planck scale, Phys. Lett. B 522 (2001), 133-138, hep-th/0107039.

[70] Cailleteau T., Mielczarek J., Barrau A., Grain J., Anomaly-free scalar perturbations with holonomy corrections in loop quantum cosmology, Classical Quantum Gravity 29 (2012), 095010, 17 pages, arXiv:1111.3535.

[71] Calcagni G., Observational effects from quantum cosmology, arXiv:1209.0473.

[72] Calcagni G., Hossain G.M., Loop quantum cosmology and tensor perturbations in the early universe, Adv. Sci. Lett. 2 (2009), 184-193, arXiv:0810.4330.

[73] Camacho A., Some consequences of a generalization to Heisenberg algebra in quantum electrodynamics, Internat. J. Modern Phys. D 12 (2003), 1687-1692, gr-qc/0305052.

[74] Camacho A., White dwarfs as test objects of Lorentz violations, Classical Quantum Gravity 23 (2006), 7355-7368, gr-qc/0610073.

[75] Carlson C.E., Carone C.D., Zobin N., Noncommutative gauge theory without Lorentz violation, Phys. Rev. D 66 (2002), 075001, 8 pages, hep-th/0206035.

[76] Chaichian M., Kulish P.P., Nishijima K., Tureanu A., On a Lorentz-invariant interpretation of noncommutative space-time and its implications on noncommutative QFT, Phys. Lett. B 604 (2004), 98-102, hep-th/0408069.

[77] Chaichian M., Prešnajder P., Sheikh-Jabbari M.M., Tureanu A., Noncommutative gauge field theories: a no-go theorem, Phys. Lett. B 526 (2002), 132-136, hep-th/0107037.

[78] Christian J., Testing quantum gravity via cosmogenic neutrino oscillations, Phys. Rev. D 71 (2005), 024012, 8 pages, gr-qc/0409077.

[79] Colladay D., Kostelecký A., Lorentz-violating extension of the standard model, Phys. Rev. D 58 (1998), 116002, 23 pages, hep-ph/9809521.

[80] Collins J., Perez A., Sudarsky D., Lorentz invariance violation and its role in quantum gravity phenomenology, in Approaches to Quantum Gravity: Toward a New Understanding of Space, Time and Matter, Editor D. Oriti, Cambridge University Press, Cambridge, 2009, 528-547, hep-th/0603002.

[81] Collins J., Perez A., Sudarsky D., Urrutia L., Vucetich H., Lorentz invariance and quantum gravity: an additional fine-tuning problem?, Phys. Rev. Lett. 93 (2004), 191301, 4 pages, gr-qc/0403053.

[82] Copeland E.J., Mulryne D.J., Nunes N.J., Shaeri M., Gravitational wave background from superinflation in loop quantum cosmology, Phys. Rev. D 79 (2009), 023508, 8 pages, arXiv:0810.0104.

[83] Corichi A., Black holes and entropy in loop quantum gravity: an overview, arXiv:0901.1302.

[84] Dapor A., Lewandowski J., Tavakoli Y., Lorentz symmetry in QFT on quantum Bianchi I space-time, Phys. Rev. D 86 (2012), 064013, 14 pages, arXiv:1207.0671.

[85] De Pietri R., Rovelli C., Geometry eigenvalues and the scalar product from recoupling theory in loop quantum gravity, Phys. Rev. D 54 (1996), 2664-2690, gr-qc/9602023.

[86] DeWitt B.S., Quantum theory of gravity. I. The canonical theory, Phys. Rev. 160 (1967), 1113-1148.

[87] Dittrich B., Thiemann T., Are the spectra of geometrical operators in loop quantum gravity really discrete?, J. Math. Phys. 50 (2009), 012503, 11 pages, arXiv:0708.1721.

[88] Doplicher S., Fredenhagen K., Roberts J.E., The quantum structure of spacetime at the Planck scale and quantum fields, Comm. Math. Phys. 172 (1995), 187-220, hep-th/0303037. 
[89] Dupuis M., Livine E.R., Lifting SU(2) spin networks to projected spin networks, Phys. Rev. D 82 (2010), 064044, 11 pages, arXiv:1008.4093.

[90] Fairbairn W.J., Livine E.R., 3D spinfoam quantum gravity: matter as a phase of the group field theory, Classical Quantum Gravity 24 (2007), 5277-5297, gr-qc/0702125.

[91] Filk T., Divergencies in a field theory on quantum space, Phys. Lett. B 376 (1996), 53-58.

[92] Fiore G., Wess J., On full twisted Poincaré symmetry and QFT on Moyal-Weyl spaces, Phys. Rev. D 75 (2007), 105022, 13 pages, hep-th/0701078.

[93] Freidel L., The geometry of momentum space, in preparation.

[94] Freidel L., Geiller M., Ziprick J., Continuous formulation of the loop quantum gravity phase space, arXiv:1110.4833.

[95] Freidel L., Kowalski-Glikman J., $\kappa$-Minkowski space, scalar field, and the issue of Lorentz invariance, arXiv:0710.2886.

[96] Freidel L., Kowalski-Glikman J., Nowak S., Field theory on $\kappa$-Minkowski space revisited: Noether charges and breaking of Lorentz symmetry, Internat. J. Modern Phys. A 23 (2008), 2687-2718, arXiv:0706.3658.

[97] Freidel L., Livine E.R., Ponzano-Regge model revisited. III. Feynman diagrams and effective field theory, Classical Quantum Gravity 23 (2006), 2021-2061, hep-th/0502106.

[98] Freidel L., Speziale S., Twisted geometries: a geometric parametrisation of SU(2) phase space, Phys. Rev. D 82 (2010), 084040, 16 pages, arXiv:1001.2748.

[99] Freidel L., Starodubtsev A., Quantum gravity in terms of topological observables, hep-th/0501191.

[100] Galaverni M., Sigl G., Lorentz violation for photons and ultrahigh-energy cosmic rays, Phys. Rev. Lett. 100 (2008), 021102, 4 pages, arXiv:0708.1737.

[101] Gambini R., Pullin J., Nonstandard optics from quantum space-time, Phys. Rev. D 59 (1999), 124021, 4 pages, gr-qc/9809038.

[102] Gambini R., Pullin J., Rastgoo S., Quantum scalar field in quantum gravity: the propagator and Lorentz invariance in the spherically symmetric case, Gen. Relativity Gravitation 43 (2011), 3569-3592, arXiv:1105.0667.

[103] Gambini R., Rastgoo S., Pullin J., Small Lorentz violations in quantum gravity: do they lead to unacceptably large effects?, Classical Quantum Gravity 28 (2011), 155005, 6 pages, arXiv:1106.1417.

[104] Giesel K., Thiemann T., Consistency check on volume and triad operator quantization in loop quantum gravity. I, Classical Quantum Gravity 23 (2006), 5667-5691, gr-qc/0507036.

[105] Giesel K., Thiemann T., Consistency check on volume and triad operator quantization in loop quantum gravity. II, Classical Quantum Gravity 23 (2006), 5693-5771, gr-qc/0507037.

[106] Girelli F., From quantum reference frames to deformed special relativity, in Approaches to Quantum Gravity: Toward a New Understanding of Space, Time and Matter, Editor D. Oriti, Cambridge University Press, Cambridge, 2009, 509-527, gr-qc/0607032.

[107] Girelli F., Snyder space-time: K-loop and Lie triple system, SIGMA 6 (2010), 074, 19 pages, arXiv:1009.4762.

[108] Girelli F., Liberati S., Percacci R., Rahmede C., Modified dispersion relations from the renormalization group of gravity, Classical Quantum Gravity 24 (2007), 3995-4008, gr-qc/0607030.

[109] Girelli F., Liberati S., Sindoni L., Planck-scale modified dispersion relations and Finsler geometry, Phys. Rev. D 75 (2007), 064015, 9 pages, gr-qc/0611024.

[110] Girelli F., Livine E.R., Physics of deformed special relativity: relativity principle revisited, gr-qc/0412004.

[111] Girelli F., Livine E.R., Scalar field theory in Snyder space-time: alternatives, J. High Energy Phys. 2011 (2011), no. 3, 132, 31 pages, arXiv:1004.0621.

[112] Girelli F., Livine E.R., Oriti D., Four-dimensional deformed special relativity from group field theories, Phys. Rev. D 81 (2010), 024015, 14 pages, arXiv:0903.3475.

[113] Girelli F., Poulin D., Quantum reference frames and deformed symmetries, Phys. Rev. D 77 (2008), 104012, 11 pages, arXiv:0710.4393.

[114] Gleiser R.J., Kozameh C.N., Astrophysical limits on quantum gravity motivated birefringence, Phys. Rev. D 64 (2001), 083007, 4 pages, gr-qc/0102093.

[115] Grain J., Barrau A., Cosmological footprints of loop quantum gravity, Phys. Rev. Lett. 102 (2009), 081301, 4 pages, arXiv:0902.0145. 
[116] Grain J., Barrau A., Cailleteau T., Mielczarek J., Observing the big bounce with tensor modes in the cosmic microwave background: phenomenology and fundamental loop quantum cosmology parameters, Phys. Rev. D 82 (2010), 123520, 12 pages, arXiv:1011.1811.

[117] Grain J., Cailleteau T., Barrau A., Gorecki A., Fully loop-quantum-cosmology-corrected propagation of gravitational waves during slow-roll inflation, Phys. Rev. D 81 (2010), 024040, 7 pages, arXiv:0910.2892.

[118] Gregg M., Major S.A., On modified dispersion relations and the Chandrasekhar mass limit, Internat. J. Modern Phys. D 18 (2009), 971-982, arXiv:0806.3496.

[119] Groenewold H.J., On the principles of elementary quantum mechanics, Physica 12 (1946), 405-460.

[120] Groot Nibbelink S., Pospelov M., Lorentz violation in supersymmetric field theories, Phys. Rev. Lett. 94 (2005), 081601, 4 pages, hep-ph/0404271.

[121] Grosse H., Wohlgenannt M., On $\kappa$-deformation and UV/IR mixing, Nuclear Phys. B 748 (2006), 473-484, hep-th/0507030.

[122] Grosse H., Wulkenhaar R., Progress in solving a noncommutative quantum field theory in four dimensions, arXiv:0909.1389.

[123] Grot N., Rovelli C., Moduli-space structure of knots with intersections, J. Math. Phys. 37 (1996), 30143021, gr-qc/9604010.

[124] Gubitosi G., Genovese G., Amelino-Camelia G., Melchiorri A., Planck-scale modifications to electrodynamics characterized by a spacelike symmetry-breaking vector, Phys. Rev. D 82 (2010), 024013, 8 pages, arXiv:1003.0878.

[125] Gubitosi G., Mercati F., Relative locality in $\kappa$-Poincaré, arXiv:1106.5710.

[126] Gurau R., Magnen J., Rivasseau V., Tanasa A., A translation-invariant renormalizable non-commutative scalar model, Comm. Math. Phys. 287 (2009), 275-290, arXiv:0802.0791.

[127] Heisenberg W., Über die in der Theorie der Elementarteilchen auftretende universelle Länge, Ann. Phys. 424 (1938), 20-33.

[128] Heyman D., Major S.A., Hinteleitner F., Reaction thresholds in doubly special relativity, Phys. Rev. D 69 (2004), 105016, 8 pages, gr-qc/0312089.

[129] Hinchliffe I., Kersting N., Ma Y.L., Review of the phenomenology of noncommutative geometry, Internat. J. Modern Phys. A 19 (2004), 179-204, hep-ph/0205040.

[130] Hinterleitner F., Remarks on doubly special relativity theories and gravity, Classical Quantum Gravity 25 (2008), 075018, 11 pages, arXiv:0706.0471.

[131] Hossenfelder S., A note on theories with a minimal length, Classical Quantum Gravity 23 (2006), 1815-1821, hep-th/0510245.

[132] Hossenfelder S., Bounds on an energy-dependent and observer-independent speed of light from violations of locality, Phys. Rev. Lett. 104 (2010), 071301, 4 pages, arXiv:1004.0418.

[133] Hossenfelder S., Interpretation of quantum field theories with a minimal length scale, Phys. Rev. D 73 (2006), 105013, 9 pages, hep-th/0603032.

[134] Hossenfelder S., Minimal length scale scenarios for quantum gravity, arXiv:1203.6191.

[135] Hossenfelder S., The box-problem in deformed special relativity, arXiv:0912.0090.

[136] Hossenfelder S., Modesto L., Prémont-Schwarz I., Emission spectra of self-dual black holes, arXiv:1202.0412.

[137] Husain V., Major S.A., Gravity and BF theory defined in bounded regions, Nuclear Phys. B 500 (1997), 381-401, gr-qc/9703043.

[138] Jacob U., Mercati F., Amelino-Camelia G., Piran T., Modifications to Lorentz invariant dispersion in relatively boosted frames, Phys. Rev. D 82 (2010), 084021, 11 pages, arXiv:1004.0575.

[139] Jacobson T., Liberati S., Mattingly D., Astrophysical bounds on Planck suppressed Lorentz violation, in Planck Scale Efffects in Astrophysics and Cosmology, Lecture Notes in Phys., Vol. 669, Springer, Berlin, 2005, 101-130, hep-ph/0407370.

[140] Jacobson T., Liberati S., Mattingly D., Threshold effects and Planck scale Lorentz violation: combined constraints from high energy astrophysics, Phys. Rev. D 67 (2003), 124011, 26 pages, hep-ph/0209264.

[141] Jacobson T., Liberati S., Mattingly D., Lorentz violation at high energy: concepts, phenomena and astrophysical constraints, Ann. Physics 321 (2006), 150-196, astro-ph/0505267.

[142] Jacobson T., Liberati S., Mattingly D., Stecker F.W., New limits on Planck scale Lorentz violation in QED, Phys. Rev. Lett. 93 (2004), 021101, 4 pages, astro-ph/0309681. 
[143] Joung E., Mourad J., Noui K., Three dimensional quantum geometry and deformed symmetry, J. Math. Phys. 50 (2009), 052503, 29 pages, arXiv:0806.4121.

[144] Jurcǒ B., Schraml S., Schupp P., Wess J., Enveloping algebra-valued gauge transformations for non-abelian gauge groups on non-commutative spaces, Eur. Phys. J. C 17 (2000), 521-526, hep-th/0006246.

[145] Kelley J.L., Searching for quantum gravity with neutrino telescopes, PoS Proc. Sci. (2007), PoS(QG-Ph), 022, 14 pages.

[146] Kempf A., Mangano G., Mann R.B., Hilbert space representation of the minimal length uncertainty relation, Phys. Rev. D 52 (1995), 1108-1118, hep-th/9412167.

[147] Kodama H., Holomorphic wave function of the Universe, Phys. Rev. D 42 (1990), 2548-2565.

[148] Kodama H., Specialization of Ashtekar's formalism to Bianchi cosmology, Progr. Theoret. Phys. 80 (1988), 1024-1040.

[149] Konopka T., Major S.A., Observational limits on quantum geometry effects, New J. Phys. 4 (2002), 57, 18 pages, hep-ph/0201184.

[150] Kostelecký A. (Editor), Proceedings of the Second Meeting on CPT and Lorentz Symmetry, World Scientific Publishing Co. Inc., River Edge, NJ, 2002.

[151] Kostelecký A., Mewes M., Electrodynamics with Lorentz-violating operators of arbitrary dimension, Phys. Rev. D 80 (2009), 015020, 59 pages, arXiv:0905.0031.

[152] Kostelecký A., Russell N., Data tables for Lorentz and CPT violation, Rev. Modern Phys. 83 (2011), 11-31, arXiv:0801.0287.

[153] Kowalski-Glikman J., Doubly special relativity: facts and prospects, in Approaches to Quantum Gravity: Toward a New Understanding of Space, Time and Matter, Editor D. Oriti, Cambridge University Press, Cambridge, 2009, 493-508, gr-qc/0603022.

[154] Kowalski-Glikman J., Planck-scale relativity from quantum $\kappa$-Poincaré algebra, Modern Phys. Lett. A 17 (2002), 1-12, hep-th/0107054.

[155] Kowalski-Glikman J., Nowak S., Doubly special relativity theories as different bases of $\kappa$-Poincaré algebra, Phys. Lett. B 539 (2002), 126-132, hep-th/0203040.

[156] Kowalski-Glikman J., Starodubtsev A., Effective particle kinematics from quantum gravity, Phys. Rev. D 78 (2008), 084039, 10 pages, arXiv:0808.2613.

[157] Kozameh C.N., Parisi F., Lorentz invariance and the semiclassical approximation of loop quantum gravity, Classical Quantum Gravity 21 (2004), 2617-2621, gr-qc/0310014.

[158] Krasnov K., Geometrical entropy from loop quantum gravity, Phys. Rev. D 55 (1997), 3505-3513.

[159] Krasnov K., The area spectrum in quantum gravity, Classical Quantum Gravity 15 (1998), L47-L53, gr-qc/9803074.

[160] Krasnov K., Rovelli C., Black holes in full quantum gravity, Classical Quantum Gravity 26 (2009), 245009, 8 pages, arXiv:0905.4916.

[161] Laurent P., Götz D., Binétruy P., Covino S., Fernandez-Soto A., Constraints on Lorentz invariance violation using integral/IBIS observations of GRB041219A, Phys. Rev. D 83 (2011), 121301, 5 pages, arXiv:1106.1068.

[162] Lewandowski J., Volume and quantizations, Classical Quantum Gravity 14 (1997), 71-76, gr-qc/9602035.

[163] Liberati S., Maccione L., Lorentz violation: motivation and new constraints, Ann. Rev. Nuclear Part. Sci. 59 (2009), 245-267, arXiv:0906.0681.

[164] Liberati S., Maccione L., Quantum gravity phenomenology: achievements and challenges, J. Phys. Conf. Ser. 314 (2011), 012007, 10 pages, arXiv:1105.6234.

[165] Liberati S., Sonego S., Visser M., Interpreting doubly special relativity as a modified theory of measurement, Phys. Rev. D 71 (2005), 045001, 9 pages, gr-qc/0410113.

[166] Livine E.R., Boucles et mousses de spin en gravite quantique: une approche covariante à la quantification non-perturbative de la relativité générale, Ph.D. thesis, Centre de Physique Theorique, 2003.

[167] Livine E.R., Oriti D., About Lorentz invariance in a discrete quantum setting, J. High Energy Phys. 2004 (2004), no. 6, 050, 36 pages, gr-qc/0405085.

[168] Loll R., Spectrum of the volume operator in quantum gravity, Nuclear Phys. B 460 (1996), 143-154, gr-qc/9511030.

[169] Loll R., Volume operator in discretized quantum gravity, Phys. Rev. Lett. 75 (1995), 3048-3051, gr-qc/9506014. 
[170] Lukierski J., Ruegg H., Nowicki A., Tolstoy V.N., q-deformation of Poincaré algebra, Phys. Lett. B 264 (1991), 331-338.

[171] Ma Y., Soo C., Yang J., New length operator for loop quantum gravity, Phys. Rev. D 81 (2010), 124026, 9 pages, arXiv:1004.1063.

[172] Maccione L., Liberati S., Celotti A., Kirk J.G., New constraints on Planck-scale Lorentz violation in QED from the Crab Nebula, J. Cosmol. Astropart. Phys. 2007 (2007), no. 10, 013, 24 pages, arXiv:0707.2673.

[173] Maccione L., Liberati S., Celotti A., Kirk J.G., Ubertini P., $\gamma$-ray polarization constraints on Planck scale violations of special relativity, Phys. Rev. D $\mathbf{7 8}$ (2008), 103003, 5 pages, arXiv:0809.0220.

[174] Maccione L., Taylor A.M., Mattingly D., Liberati S., Planck-scale Lorentz violation constrained by ultrahigh-energy cosmic rays, J. Cosmol. Astropart. Phys. 2009 (2009), no. 4, 022, 21 pages, arXiv:0902.1756.

[175] Maggiore M., Quantum groups, gravity, and the generalized uncertainty principle, Phys. Rev. D 49 (1994), 5182-5187, hep-th/9305163.

[176] Maggiore M., The algebraic structure of the generalized uncertainty principle, Phys. Lett. B 319 (1993), 83-86, hep-th/9309034.

[177] Magueijo J., Benincasa D.M.T., Chiral vacuum fluctuations in quantum gravity, Phys. Rev. Lett. 106 (2011), 121302, 4 pages, arXiv:1010.3552.

[178] Magueijo J., Bethke L., New ground state for quantum gravity, arXiv:1207.0637.

[179] Magueijo J., Smolin L., Generalized Lorentz invariance with an invariant energy scale, Phys. Rev. D 67 (2003), 044017, 12 pages, gr-qc/0207085.

[180] Magueijo J., Smolin L., Gravity's rainbow, Classical Quantum Gravity 21 (2004), 1725-1736, gr-qc/0305055.

[181] Magueijo J., Smolin L., Lorentz invariance with an invariant energy scale, Phys. Rev. Lett. 88 (2002), 190403, 4 pages, hep-th/0112090.

[182] Majid S., Foundations of quantum group theory, Cambridge University Press, Cambridge, 1995.

[183] Majid S., On q-regularization, Internat. J. Modern Phys. A 5 (1990), 4689-4696.

[184] Majid S., Ruegg H., Bicrossproduct structure of $\kappa$-Poincaré group and non-commutative geometry, Phys. Lett. B 334 (1994), 348-354, hep-th/9405107.

[185] Major S.A., Embedded graph invariants in Chern-Simons theory, Nuclear Phys. B 550 (1999), 531-560, hep-th/9810071.

[186] Major S.A., On the q-quantum gravity loop algebra, Classical Quantum Gravity 25 (2008), 065003, 9 pages, arXiv:0708.0750.

[187] Major S.A., Operators for quantized directions, Classical Quantum Gravity 16 (1999), 3859-3877, gr-qc/9905019.

[188] Major S.A., q-quantum gravity, Ph.D. thesis, The Pennsylvania State University, 1997.

[189] Major S.A., Quantum geometry phenomenology: angle and semiclassical states, J. Phys. Conf. Ser. 360 (2012), 012061, 4 pages, arXiv:1112.4366.

[190] Major S.A., Shape in an atom of space: exploring quantum geometry phenomenology, Classical Quantum Gravity 27 (2010), 225012, 16 pages, arXiv:1005.5460.

[191] Major S.A., Seifert M.D., Modelling space with an atom of quantum geometry, Classical Quantum Gravity 19 (2002), 2211-2227, gr-qc/0109056.

[192] Major S.A., Smolin L., Quantum deformation of quantum gravity, Nuclear Phys. B 473 (1996), 267-290, gr-qc/9512020.

[193] Mattingly D., Have we tested Lorentz invariance enough?, arXiv:0802.1561.

[194] Mattingly D., Modern tests of Lorentz invariance, Living Rev. Relativity 8 (2005), 5, 84 pages, gr-qc/0502097.

[195] Mattingly D.M., Maccione L., Galaverni M., Liberati S., Sigl G., Possible cosmogenic neutrino constraints on Planck-scale Lorentz violation, J. Cosmol. Astropart. Phys. 2010 (2010), no. 2, 007, 20 pages, arXiv:0911.0521.

[196] Matusis A., Susskind L., Toumbas N., The IR/UV connection in the noncommutative gauge theories, J. High Energy Phys. 2000 (2000), no. 12, 002, 18 pages, hep-th/0002075.

[197] Meissner K.A., Black-hole entropy in loop quantum gravity, Classical Quantum Gravity 21 (2004), 52455251, gr-qc/0407052. 
[198] Meissner K.A., Eigenvalues of the volume operator in loop quantum gravity, Classical Quantum Gravity 23 (2006), 617-625, gr-qc/0509049.

[199] Meusburger C., Schroers B.J., Generalised Chern-Simons actions for 3d gravity and $\kappa$-Poincaré symmetry, Nuclear Phys. B 806 (2009), 462-488, arXiv:0805.3318.

[200] Mielczarek J., Gravitational waves from the big bounce, J. Cosmol. Astropart. Phys. 2008 (2008), no. 11, 011, 17 pages, arXiv:0807.0712.

[201] Mielczarek J., Possible observational effects of loop quantum cosmology, Phys. Rev. D 81 (2010), 063503, 12 pages, arXiv:0908.4329.

[202] Mielczarek J., Tensor power spectrum with holonomy corrections in LQC, Phys. Rev. D 79 (2009), 123520, 13 pages, arXiv:0902.2490.

[203] Mielczarek J., Cailleteau T., Barrau A., Grain J., Anomaly-free vector perturbations with holonomy corrections in loop quantum cosmology, arXiv:1106.3744.

[204] Mielczarek J., Cailleteau T., Grain J., Barrau A., Inflation in loop quantum cosmology: dynamics and spectrum of gravitational waves, Phys. Rev. D $\mathbf{8 1}$ (2010), 104049, 11 pages, arXiv:1003.4660.

[205] Minwalla S., Van Raamsdonk M., Seiberg N., Noncommutative perturbative dynamics, J. High Energy Phys. 2000 (2000), no. 2, 020, 31 pages, hep-th/9912072.

[206] Modesto L., Prémont-Schwarz I., Self-dual black holes in LQG: theory and phenomenology, Phys. Rev. D 80 (2009), 064041, 17 pages, arXiv:0905.3170.

[207] Moyal J.E., Quantum mechanics as a statistical theory, Proc. Cambridge Philos. Soc. 45 (1949), 99-124.

[208] Myers R.C., Pospelov M., Ultraviolet modifications of dispersion relations in effective field theory, Phys. Rev. Lett. 90 (2003), 211601, 4 pages, hep-ph/0301124.

[209] Oeckl R., Braided quantum field theory, Comm. Math. Phys. 217 (2001), 451-473, hep-th/9906225.

[210] Oeckl R., Untwisting noncommutative $\mathbf{R}^{d}$ and the equivalence of quantum field theories, Nuclear Phys. B 581 (2000), 559-574, hep-th/0003018.

[211] Percival I.C., Strunz W.T., Detection of spacetime fluctuations by a model interferometer, Proc. Roy. Soc. London. Ser. A 453 (1997), 431-446, quant-ph/9607011.

[212] Perez A., Introduction to loop quantum gravity and spin foams, gr-qc/0409061.

[213] Pfeifer C., Wohlfarth M.N.R., Causal structure and electrodynamics on Finsler spacetimes, Phys. Rev. D 84 (2011), 044039, 14 pages, arXiv:1104.1079.

[214] Pinzul A., Twisted Poincaré group and spin-statistics, Internat. J. Modern Phys. A 20 (2005), 6268-6277.

[215] Podleś P., Woronowicz S.L., On the classification of quantum Poincaré groups, Comm. Math. Phys. 178 (1996), 61-82, hep-th/9412059.

[216] Polchinski J., Comment on "Small Lorentz violations in quantum gravity: do they lead to unacceptably large effects?", arXiv:1106.6346.

[217] Poulin D., Yard J., Dynamics of a quantum reference frame, New J. Phys. 9 (2007), no. 5, 156,17 pages, quant-ph/0612126.

[218] Randono A.C., In search of quantum de Sitter space: generalizing the Kodama state, Ph.D. thesis, University of Texas at Austin, 2007, arXiv:0709.2905.

[219] Rätzel D., Rivera S., Schuller F.P., Geometry of physical dispersion relations, Phys. Rev. D 83 (2011), 044047, 23 pages, arXiv:1010.1369.

[220] Rodríguez-Romo S., Some examples of q-regularization, Internat. J. Theoret. Phys. 34 (1995), 2179-2193, hep-th/9508019.

[221] Roth M. et al. (Auger Collaboration), Measurement of the UHECR energy spectrum using data from the surface detector of the Pierre Auger observatory, arXiv:0706.2096.

[222] Rovelli C., A generally covariant quantum field theory and a prediction on quantum measurements of geometry, Nuclear Phys. B 405 (1993), 797-815.

[223] Rovelli C., A new look at loop quantum gravity, Classical Quantum Gravity 28 (2011), 114005, 24 pages, arXiv:1004.1780.

[224] Rovelli C., Black hole entropy from loop quantum gravity, Phys. Rev. Lett. 77 (1996), 3288-3291, gr-qc/9603063. 
[225] Rovelli C., Comment on "Are the spectra of geometrical operators in loop quantum gravity really discrete?" by B. Dittrich and T. Thiemann, arXiv:0708.2481.

[226] Rovelli C., Quantum gravity, Cambridge Monographs on Mathematical Physics, Cambridge University Press, Cambridge, 2004.

[227] Rovelli C., Zakopane lectures on loop gravity, arXiv:1102.3660.

[228] Rovelli C., Smolin L., Discreteness of area and volume in quantum gravity, Nuclear Phys. B 442 (1995), 593-619, Erratum, Nuclear Phys. B 456 (1995), 753-754, gr-qc/9411005.

[229] Rovelli C., Speziale S., Lorentz covariance of loop quantum gravity, Phys. Rev. D 83 (2011), 104029, 6 pages, arXiv:1012.1739.

[230] Rovelli C., Speziale S., Reconcile Planck-scale discreteness and the Lorentz-Fitzgerald contraction, Phys. Rev. D 67 (2003), 064019, 11 pages, gr-qc/0205108.

[231] Rubtsov G.I. et al., Upper limit on the ultrahigh-energy photon flux from AGASA and Yakutsk data, Phys. Rev. D 73 (2006), 063009, 8 pages, astro-ph/0601449.

[232] Sahlmann H., Loop quantum gravity - a short review, arXiv:1001.4188.

[233] Sahlmann H., Thiemann T., Towards the QFT on curved spacetime limit of QGR. I. A general scheme, Classical Quantum Gravity 23 (2006), 867-908, gr-qc/0207030.

[234] Sahlmann H., Thiemann T., Towards the QFT on curved spacetime limit of QGR. II. A concrete implementation, Classical Quantum Gravity 23 (2006), 909-954, gr-qc/0207031.

[235] Sakellariadou M., Lattice refinement in loop quantum cosmology, J. Phys. Conf. Ser. 189 (2009), 012035, 17 pages, arXiv:0810.5356.

[236] Sakellariadou M., Phenomenology of loop quantum cosmology, J. Phys. Conf. Ser. 222 (2010), 012027, 16 pages, arXiv:1001.0161.

[237] Saveliev A., Maccione L., Sigl G., Lorentz invariance violation and chemical composition of ultra high energy cosmic rays, J. Cosmol. Astropart. Phys. 2011 (2011), no. 3, 046, 18 pages, arXiv:1101.2903.

[238] Schuller F.P., All spacetimes beyond Einstein (Obergurgl lectures), arXiv:1111.4824.

[239] Seifert M., Angle and volume studies in quantized space, B.A. Thesis, Swarthmore College, Swarthmore, 2001, gr-qc/0108047.

[240] Sheikh-Jabbari M.M., Renormalizability of the supersymmetric Yang-Mills theories on the noncommutative torus, J. High Energy Phys. 1999 (1999), no. 6, 015, 16 pages, hep-th/9903107.

[241] Sitarz A., Noncommutative differential calculus on the $\kappa$-Minkowski space, Phys. Lett. B 349 (1995), 42-48, hep-th/9409014.

[242] Skákala J., Visser M., Pseudo-Finslerian space-times and multirefringence, Internat. J. Modern Phys. D 19 (2010), 1119-1146, arXiv:0806.0950.

[243] Smolin L., Classical paradoxes of locality and their possible quantum resolutions in deformed special relativity, Gen. Relativity Gravitation 43 (2011), 3671-3691, arXiv:1004.0664.

[244] Smolin L., Could deformed special relativity naturally arise from the semiclassical limit of quantum gravity?, arXiv:0808.3765.

[245] Smolin L., Falsifiable predictions from semiclassical quantum gravity, Nuclear Phys. B 742 (2006), 142-157, hep-th/0501091.

[246] Smolin L., On limitations of the extent of inertial frames in non-commutative spacetimes, arXiv:1007.0718.

[247] Smolin L., Quantum gravity with a positive cosmological constant, hep-th/0209079.

[248] Smolin L., Soo C., The Chern-Simons invariant as the natural time variable for classical and quantum cosmology, Nuclear Phys. B 449 (1995), 289-314, gr-qc/9405015.

[249] Smolin L., Starodubtsev A., General relativity with a topological phase: an action principle, hep-th/0311163.

[250] Snyder H.S., Quantized space-time, Phys. Rev. 71 (1947), 38-41.

[251] Starodubtsev A., Topological excitations around the vacuum of quantum gravity I: The symmetries of the vacuum, hep-th/0306135.

[252] Stecker F.W., A new limit on Planck scale Lorentz violation from $\gamma$-ray burst polarization, Astropart. Phys. 35 (2011), 95-97, arXiv:1102.2784. 
[253] Thiemann T., A length operator for canonical quantum gravity, J. Math. Phys. 39 (1998), 3372-3392, gr-qc/9606092.

[254] Thiemann T., Closed formula for the matrix elements of the volume operator in canonical quantum gravity, J. Math. Phys. 39 (1998), 3347-3371, gr-qc/9606091.

[255] Thiemann T., Lectures on loop quantum gravity, in Quantum Gravity, Lecture Notes in Phys., Vol. 631, Springer, Berlin, 2003, 41-135, gr-qc/0210094.

[256] Thiemann T., Loop quantum gravity: an inside view, in Approaches to Fundamental Physics, Lecture Notes in Phys., Vol. 721, Springer, Berlin, 2007, 185-263, hep-th/0608210.

[257] Thiemann T., Modern canonical quantum general relativity, Cambridge Monographs on Mathematical Physics, Cambridge University Press, Cambridge, 2007.

[258] Thiemann T., Quantum spin dynamics (QSD), Classical Quantum Gravity 15 (1998), 839-873, gr-qc/9606089.

[259] Ungar A.A., Thomas precession and its associated grouplike structure, Amer. J. Phys. 59 (1991), 824-834.

[260] Wang C.H.T., Bingham R., Mendonça J.T., Quantum gravitational decoherence of matter waves, Classical Quantum Gravity 23 (2006), L59-L65, gr-qc/0603112.

[261] Wilson-Ewing E., Holonomy corrections in the effective equations for scalar mode perturbations in loop quantum cosmology, Classical Quantum Gravity 29 (2012), 085005, 19 pages, arXiv:1108.6265.

[262] Wu J., Ling Y., The cosmological perturbation theory in loop cosmology with holonomy corrections, J. Cosmol. Astropart. Phys. 2010 (2010), no. 5, 026, 15 pages, arXiv:1001.1227.

[263] Yonetoku D. et al., Detection of gamma-ray polarization in prompt emission of GRB 100826A, Astrophys. J. Lett. 743 (2011), L30-L34, arXiv:1111.1779.

[264] Young C.A.S., Zegers R., Covariant particle statistics and intertwiners of the $\kappa$-deformed Poincaré algebra, Nuclear Phys. B $\mathbf{7 9 7}$ (2008), 537-549, arXiv:0711.2206.

[265] Young C.A.S., Zegers R., On $\kappa$-deformation and triangular quasibialgebra structure, Nuclear Phys. B 809 (2009), 439-451, arXiv:0807.2745.

[266] Zapata J.A., A combinatorial approach to diffeomorphism invariant quantum gauge theories, J. Math. Phys. 38 (1997), 5663-5681, gr-qc/9703037.

[267] Zapata J.A., Combinatorial space from loop quantum gravity, Gen. Relativity Gravitation 30 (1998), 12291245 , gr-qc/9703038. 